\title{
Intercalibration of the ATLAS Calorimeters and Cross Section Measurements for Inclusive Jet and Dijet Production in Proton-Proton Collisions at $\sqrt{s}=7 \mathrm{TeV}$
}

by

\author{
Miro Vujicic \\ A thesis submitted to the \\ Faculty of Graduate and Postdoctoral Affairs \\ in partial fulfillment of the requirements \\ for the degree of \\ Master of Science
}

Ottawa-Carleton Institute for Physics

Department of Physics

Carleton University

Ottawa, Ontario, Canada

September, 2011

(C)copyright

Miro Vujicic, 2011 
Library and Archives

Canada

Published Heritage

Branch

395 Wellington Street

Ottawa ON K1A ON4

Canada
Bibliothèque et

Archives Canada

Direction du

Patrimoine de l'édition

395, rue Wellington

Ottawa ON K1A ON4

Canada
Your file Votre référence

ISBN: 978-0-494-83136-6

Our file Notre référence

ISBN: 978-0-494-83136-6
NOTICE:

The author has granted a nonexclusive license allowing Library and Archives Canada to reproduce, publish, archive, preserve, conserve, communicate to the public by telecommunication or on the Internet, loan, distribute and sell theses worldwide, for commercial or noncommercial purposes, in microform, paper, electronic and/or any other formats.

The author retains copyright ownership and moral rights in this thesis. Neither the thesis nor substantial extracts from it may be printed or otherwise reproduced without the author's permission.
AVIS:

L'auteur a accordé une licence non exclusive permettant à la Bibliothèque et Archives Canada de reproduire, publier, archiver, sauvegarder, conserver, transmettre au public par télécommunication ou par l'Internet, prêter, distribuer et vendre des thèses partout dans le monde, à des fins commerciales ou autres, sur support microforme, papier, électronique et/ou autres formats.

L'auteur conserve la propriété du droit d'auteur et des droits moraux qui protège cette thèse. $\mathrm{Ni}$ la thèse ni des extraits substantiels de celle-ci ne doivent être imprimés ou autrement reproduits sans son autorisation.
In compliance with the Canadian Privacy Act some supporting forms may have been removed from this thesis.

While these forms may be included in the document page count, their removal does not represent any loss of content from the thesis.
Conformément à la loi canadienne sur la protection de la vie privée, quelques formulaires secondaires ont été enlevés de cette thèse.

Bien que ces formulaires aient inclus dans la pagination, il n'y aura aucun contenu manquant.

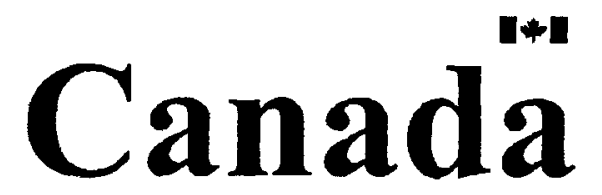




\section{Abstract}

In 2010 the Large Hadron Collider (LHC) at CERN began colliding protons at an unprecedented center-of-mass energy of $7 \mathrm{TeV}$ and roughly $37 \mathrm{pb}^{-1}$ of data have been collected by the ATLAS detector. The early data serve as an essential tool for detector commissioning which in turn allows for physics measurements to be made. The performance of the ATLAS calorimeters is investigated using real dijet events from proton-proton collisions. The uncertainty on the Jet Energy Scale is derived and is found to be largest in the most forward regions of the detector where it is roughly 13\%. In addition, the theory of the strong interaction, Quantum Chromodynamics (QCD), is put to stringent testing with all new experimental results. Measurements of the inclusive jet and dijet cross sections as functions of transverse momentum and dijet mass, respectively, are presented and compared to QCD theoretical predictions. Good agreement between data and theory is observed across most regions of phase space, except in the very forward rapidities, and in the high mass domain where the discrepancy is about $80 \%$. 


\section{Statement of Originality}

The work presented in this thesis was a collaborative effort of many people. The work was done by the ATLAS Collaboration during the first run of the LHC protonproton collisions at $\sqrt{s}=7 \mathrm{TeV}$. It contains two major parts (analyses) to which I have made significant contributions. One analysis deals with detector performance, while the other is a physics analysis.

The detector performance analysis involves the jet energy scale, its derivation and assessment. This is presented in Chapters 5, 6, and 7. In Chapter 5, the jet energy scale calibration is presented and all plots shown are taken from an internal ATLAS note [1] of which I am a main author. Chapter 6 presents the pseudorapidity intercalibration using dijet balance used for estimating the jet energy scale uncertainty. My contribution here was to run over the collected data and generate the diagnostic histograms that would illustrate the performance of the jet energy calibration. This work is presented in Chapter 6 and all the plots shown there are my own. The following chapter summarizes the full jet energy scale uncertainty and uses the results of the intercalibration presented in Chapter 6. All plots shown in Chapter 7 are also taken from the internal ATLAS note [1].

The physics analysis is the inclusive jet and dijet cross section measurement found in Chapter 8. My contribution was the derivation of the systematic uncertainty on the inclusive jet cross section arising from the uncertainty on the jet energy scale. My contribution is presented in Section 8.5.2 and all the plots shown in that section were produced by me. All other plots in Chapter 8 , unless stated otherwise, are taken from an ATLAS note [2] on which I am listed as a main author. 


\section{Acknowledgements}

Studying physics at Carleton University has truly been a wonderful experience, one that began six years ago with the undergraduate program. Some truly wonderful people are in this department, and I never really got to thank those who have taught me and show them my appreciation for all that they do. So I'll take this opportunity to mention those individuals whom I've had the chance to work closely with

and from whom I've learned a great deal from: Alain Bellerive, Pat Kalyniak, Igor Ivanovic, Bruce Campbell, Peter Watson, Steve Godfrey, Kevin Graham, Heather Logan, Manuella Vincter, Gerald Oakham, and Madhu Dixit. Some lectures that I attended taught by these professors were really awe-inspiring and memorable.

Now on to the master's thesis. I certainly could not have done all of this work without the help of my colleagues. First I must thank everyone from the ATLAS Collaboration and the people responsible for operating the LHC. They do most of the hard work, I just analyse the data. But data analysis itself can be quite challenging, and I must thank some specific individuals for their help and contributions in this regard. Thanks to Dag Gillberg, Frederick Ruhr, and Andy Pilkington for their leadership, supervision, and support with the eta intercalibration analysis. Also thanks to James Robinson and Serena Psoroulas for their help in this. Thanks to Caterina Doglioni for all her help with the jet energy scale analysis. Thanks to Pier-Olivier DeViveiros for his help in getting me started with jet studies and providing me with the nice diagram of clusters.

The cross section measurement was a huge effort of 36 individuals. Many thanks to S. Baker, G. Brown, M. Campanelli, T. Carli, V. Cavasinni, R. Chislett, C. Doglioni, 
S. Eckweiler, E. Feng, P. Francavilla, D. Gillberg, E. Hughes, G. Jones, J. Keung, P. Krieger, H. Li, B. Malaescu, C. Meyer, F. Muller, K. Perez, J. Pilcher, A. Pilkington, J. Poveda, J. Robinson, C. Roda, N. Ruthmann, B. Salvachua, S. Shimizu, P. Starovoitov, T. Sumida, K. Terashi, P. Thompson, F. Vives, S. L. Wu, and S. Yanush for their contributions.

I would like to thank my office mates at Carleton: Adam Robichaud, Rick Ueno, Thomas McCarthy, Louise Heelan, Kate Whalen, and Joshua Turner for all their help and support. Thanks to Rick for providing me with the latex thesis template and helping me with it whenever I ran into trouble. Thanks to Yun-Ha Shin, Stephen Wang, and Bill Jack from the Carleton Physics Department for their much needed help with computer hardware and software.

I want to thank my advisor, Alain Bellerive, for supporting me and providing me with the opportunity to undertake this wonderful journey. Alain is a terrific supervisor and mentor. Even though he was caught-up with multiple projects, from completely different experiments, he was still able to provide much needed guidance and keep me on track. I must also thank him for reading over the thesis and helping to make it more clear and readable. In addition, Alain has made this experience so much more enjoyable. Thanks Alain!

And last, but not least, and in fact most, I would like to thank Dag Gillberg. His name has already come up above, but he deserves full credit. Dag has been there from the beginning, helping me every step of the way. All the work that I did, Dag had some contribution to. I particularly appreciate his efforts and patience in carefully explaining all the necessary details of some of the more complex and interesting issues. This thesis really would not be what it is were it not for Dag. 


\section{Contents}

Abstract

Statement of Originality $\quad$ iii

Acknowledgments iv

$\begin{array}{ll}\text { List of Tables } & \text { ix }\end{array}$

List of Figures $\quad x$

1 Introduction 1

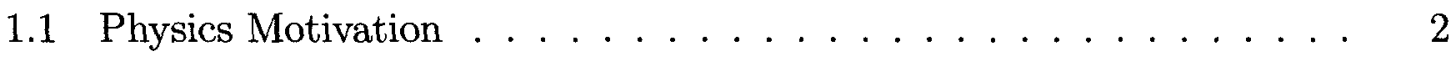

1.1.1 Standard Model . . . . . . . . . . . . . . . . . 2

1.1.2 Beyond the Standard Model . . . . . . . . . . . . 4

2 Theoretical Background: Jets and QCD 7

2.1 Overview of the Strong Interaction $\ldots \ldots \ldots \ldots$

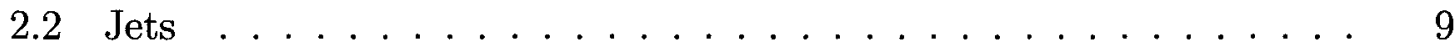

2.3 Parton Distribution Functions (PDFs) $\ldots \ldots \ldots \ldots$

2.4 Hard Scatter and the Underlying Event . . . . . . . . . . . . . 11

3 Experimental Setup $\quad 12$

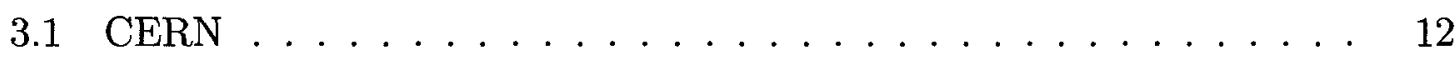

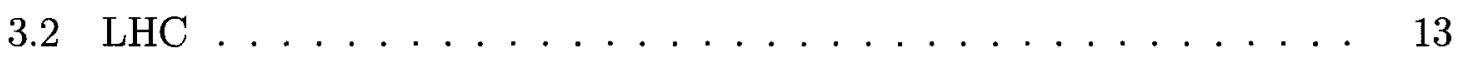

3.3 The ATLAS Detector . . . . . . . . . . . . . . . . . . . 14

3.3.1 Coordinate Systems and Common Variables . . . . . . . 15 
3.3.2 Inner Detector and Tracking . . . . . . . . . . . . . 17

3.3 .3 Calorimeters . . . . . . . . . . . . . . . . . . . . 19

3.3.4 Muon Detectors . . . . . . . . . . . . . . . . . . 22

3.3.5 Trigger System and Data Acquisition . . . . . . . . . . 24

4 Calorimetry and Particle Showering $\quad 27$

4.1 Calorimeter Showers . . . . . . . . . . . . . . . . 27

4.1.1 Electromagnetic Showers . . . . . . . . . . . . . 28

4.1 .2 Hadronic Showers . . . . . . . . . . . . . . . . . . 30

5 Jet Reconstruction in ATLAS $\quad 34$

5.1 Introduction . . . . . . . . . . . . . . . . 34

5.2 Jet Reconstruction Input Objects . . . . . . . . . . . . . . 35

5.3 The Anti- $k_{T}$ Jet Algorithm . . . . . . . . . . . . . . . . . 35

5.4 Jet Calibration $\ldots \ldots \ldots \ldots \ldots$

5.4.1 Energy Calibration Constants . . . . . . . . . . . . . 39

5.4 .2 Pseudorapidity Calibration Constants . . . . . . . . . . 40

5.5 Data-Driven Corrections . . . . . . . . . . . . . . . . . 43

5.5 .1 Pile Up . . . . . . . . . . . . . . . 43

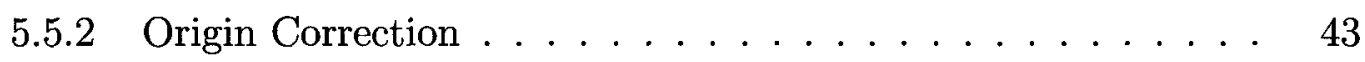

5.6 Summary . . . . . . . . . . . . . . . . . . . . . 44

6 Intercalibration Using Dijet Balance $\quad 45$

6.1 Introduction . . . . . . . . . . . . . . . . . . 45

6.2 Dijet Balance Method . . . . . . . . . . . . . . . 46

6.3 Event Selection . . . . . . . . . . . . . . . . . 47

6.3.1 Data Pre-Selection . . . . . . . . . . . . . 4 47

6.3 .2 Jet Selection . . . . . . . . . . . . . . . . . . 48

6.4 Trigger Bias and Correction $\ldots \ldots \ldots \ldots . \ldots 49$

6.5 Monte Carlo Event Simulation . . . . . . . . . . . . . . . . 51

6.6 Results . . . . . . . . . . . . . . . . . . . 51

6.7 Studies of the Underlying Event Modelling . . . . . . . . . . 56 
7 Jet Energy Scale Systematic Uncertainty $\quad 59$

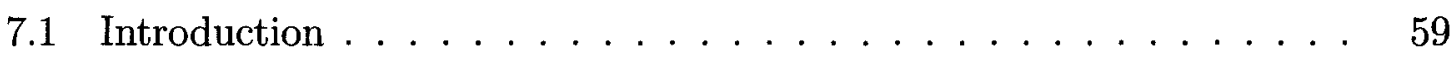

7.2 Non-Closure from JES Calibration Constants . . . . . . . . . . . 60

7.3 Uncertainties in MC Modelling . . . . . . . . . . . . . . 62

7.4 Uncertainties due to Noise Thresholds . . . . . . . . . . . . . . 65

7.5 Single Particle Tracking $\ldots \ldots \ldots \ldots 6$

7.6 Uncertainties from Material Description $\ldots \ldots \ldots \ldots$. . . . . 67

7.7 Uncertainty due to Multiple Interactions . . . . . . . . . . . . . 69

7.8 Total JES Uncertainty . . . . . . . . . . . . . . . . . . . . 73

8 Inclusive Jet and Dijet Cross Section Measurements $\quad 78$

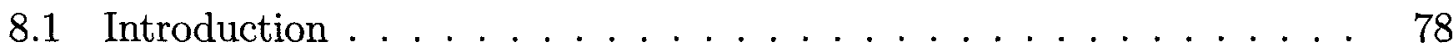

8.2 Cross Section Definition $\ldots \ldots \ldots \ldots \ldots$

8.3 Theoretical Predictions . . . . . . . . . . . . . . . . . . . 81

8.3.1 Fixed Order NLO pQCD + Non-Perturbative Corrections . . 82

8.3.2 NLO ME + Parton Shower . . . . . . . . . . . . 85

8.4 Data Selection . . . . . . . . . . . . . . . . . 86

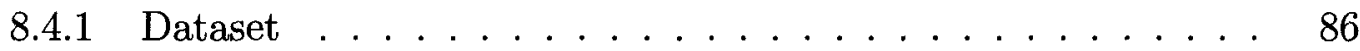

8.4 .2 Trigger $\ldots \ldots \ldots \ldots \ldots \ldots \ldots \ldots \ldots$

8.4 .3 Background Studies . . . . . . . . . . . . . . . . . 90

8.4.4 Effects of the Vertex Position . . . . . . . . . . . . . . 91

8.5 Data Correction and Measurement Uncertainties . . . . . . . . . . 93

8.5.1 Data Unfolding . . . . . . . . . . . . . . . . . 93

8.5.2 Estimation of the Uncertainty due to JES . . . . . . . . . 95

8.6 Results and Discussion . . . . . . . . . . . . . . . . . . 104

8.6.1 Inclusive Jet Cross Sections . . . . . . . . . . . . . . . . . 104

8.6 .2 Dijet Cross Sections . . . . . . . . . . . . . . . . . 121

9 Summary and Conclusion $\quad 133$

$\begin{array}{ll}\text { References } & 135\end{array}$ 


\section{List of Tables}

7.1 Summary of the maximum EM+JES jet energy scale systematic uncertainties for different $p_{\mathrm{T}}^{\mathrm{jet}}$ and $\eta$ regions from Monte Carlo-based study for anti- $k_{T}$ jets with $R=0.6 \ldots \ldots \ldots \ldots \ldots \ldots \ldots$

7.2 Summary of the maximum EM+JES jet energy scale systematic uncertainties for different $p_{\mathrm{T}}^{\text {jet }}$ and $\eta$ regions from Monte Carlo based study for anti- $k_{T}$ jets with $R=0.4 . \ldots \ldots \ldots \ldots \ldots$ 


\section{List of Figures}

1.1 The elementary particles of the Standard Model. Fermilab National Accelerator Laboratory (C). http://www.fnal.gov/pub/presspass/images/LighterHiggs-images.html. 3

3.1 Schematic of the ATLAS Detector. ATLAS Experiment (C) 2011 CERN.

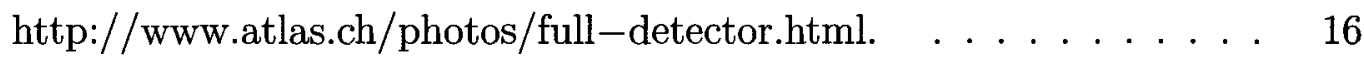

3.2 Schematic of the ATLAS Inner Detector. ATLAS Experiment (c) 2011 CERN. http://www.atlas.ch/photos/inner-detector.html. . . . . 18

3.3 Schematic of the ATLAS calorimeters. ATLAS Experiment (C) 2011 CERN. http://www.atlas.ch/photos/calorimeters.html. . . . . . . 20

3.4 Schematic of the ATLAS Forward Calorimeter. Image taken from Ref-

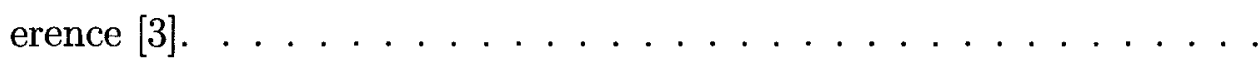

4.1 A simple depiction of a hadronic shower, highlighting the possible ways in which energy can be dissipated. Refer to text for more details. Image courtesy of Sven Menke, ATLAS Collaboration. . . . . . . . . . . .

5.1 2-Dimensional illustration of cluster formation, in an $(\eta, \phi)$ map, from individual cells in the ATLAS calorimeter. The coloured cells in the middle form a cluster while the dark grey at the bottom left do not. Image adapted from P.O. DeViveiros, ATLAS Collaboration. . . . . . 36

5.2 Simulated jet energy response in the ATLAS calorimeters as function of jet $\eta$ in different bins of energy. The shown intervals in $|\eta|$ correspond to those used in the jet energy scale uncertainty discussed in Chapter 7. 40 
5.3 Average Jet Energy Scale correction as a function of calibrated jet transverse momentum. The correction is only shown over the accessible kinematic range. . . . . . . . . . . . . . .

5.4 Average jet pseudorapidity correction constants as function of uncorrected (detector) jet $\eta \ldots \ldots \ldots \ldots \ldots \ldots$

6.1 Asymmetry distributions for the anti $-k_{T}, \mathrm{R}=0.6$ jets. Shown are several pseudorapidity bins in the $50-60 \mathrm{GeV} p_{T}^{a v g}$ bin. . . . . . . .

6.2 Asymmetry distributions for the anti $-k_{T}, \mathrm{R}=0.6$ jets. Shown are several $p_{T}^{a v g}$ bins in the 3.6-4.4 pseudorapidity (FCal) bin. . . . . . . . .

6.3 Relative jet response as a function of probe jet $\eta$ in different bins of $p_{T}^{a v g}$. The results are shown for anti $-k_{T}, \mathrm{R}=0.6$ jets. $\ldots \ldots \ldots$

6.4 Relative jet response as a function of $p_{T}^{a v g}$ in different bins of probe jet $\eta$. The results are shown for anti $-k_{T}, \mathrm{R}=0.6$ jets. $\ldots \ldots \ldots 56$

6.5 The sum of the transverse energy of all clusters in a given slice of pseudorapidity as a function of $\eta$. The $\eta$ slices have width of 0.4 . The date results, collected by the minimum bias trigger, are compared to two distinct Monte Carlo generators, Pythia and Herwig. . . . . . . .

7.1 Simulated jet $p_{T}$ response (full circles) after the EM+JES calibration and jet energy response (open squares) as a function of $p_{\mathrm{T}}^{\text {jet }}$ for the nominal MC sample for jets in the central (a), endcap (b), and forward (c) calorimeter regions. . . . . . . . . . . . . . . . . .

7.2 Simulated energy response (a) and $p_{\mathrm{T}}^{\text {jet }}$ response (b) as a function of $p_{\mathrm{T}}^{\text {jet }}$ in the central region $(0.3<|\eta|<0.8)$ for Alpgen+Herwig+Jimmy (open squares) and Pythia with the Perugia2010 tune (full triangles). The response of the nominal Monte Carlo sample is shown for comparison (full circles) . . . . . . . . . . . . . . . . .

7.3 Simulated (a) jet energy response and (b) jet $p_{T}$ response as a function of $p_{\mathrm{T}}^{\text {jet }}$ in the central region $(0.3<|\eta|<0.8)$ in the case of additional dead material in the inner detector (full triangles) and in both the inner detector and the calorimeters (open squares). The response within the nominal Monte Carlo sample is shown for comparison (full circles). . 
7.4 Relative pile-up uncertainty for anti- $k_{T}$ jets with $\mathrm{R}=0.6$ in the case of two measured primary vertices, $N_{\mathrm{PV}}=2$, for central $(0.3<|\eta|<0.8$, full circles), endcap $(2.1<|\eta| \leq 2.8$, open squares) and forward (3.6< $|\eta| \leq 4.5$, full triangles) jets as a function of jet $p_{T} . \quad \ldots \ldots \ldots \ldots$

7.5 Fractional jet energy scale systematic uncertainty as a function of $p_{\mathrm{T}}^{\text {jet }}$ for jets in the pseudorapidity region $0.3 \leq|\eta|<0.8$ in the calorimeter barrel. The total uncertainty is shown as the solid light blue area. The individual sources are also shown, with uncertainties from the fitting procedure if applicable. . . . . . . . . . . . . . . .

7.6 Fractional jet energy scale systematic uncertainty as a function of $p_{\mathrm{T}}^{\text {jet }}$ for jets in the pseudorapidity region $2.1 \leq|\eta|<2.8$. The JES uncertainty in the endcap region is extrapolated from the barrel uncertainty, with the uncertainty contribution from the $\eta$ intercalibration between central and endcap jets in data and Monte Carlo added in quadrature. The total uncertainty is shown as the solid light blue area. The individual sources are also shown, with uncertainties from the fitting procedure if applicable. . . . . . . . . . . . .

7.7 Fractional jet energy scale systematic uncertainty as a function of $p_{\mathrm{T}}^{\text {jet }}$ for jets in the pseudorapidity region $3.6<|\eta|<4.5$. The JES uncertainty for the forward region is extrapolated from the barrel uncertainty, with the uncertainty contribution from the $\eta$ intercalibration between central and forward jets in data and Monte Carlo added in quadrature. The total uncertainty is shown as the solid light blue area. The individual sources are also shown, with uncertainties from the fitting procedure if applicable. . . . . . . . . . . .

8.1 The three stages of the QCD calculation process. PDFs are needed to select specific partons within the protons to take part in the hard scatter. The hard scatter involves gluon exchange between two partons and is a perturbative calculation. The last step hadronizes the partons such that the calculation is valid for final state jets. Image adapted from T.J. LeCompte, ATLAS Collaboration. . . . . . . . . . . . . 
8.2 Integrated luminosity versus week. Plot taken from the ATLAS public results page: http://atlas.web.cern.ch/Atlas/Collaboration/. . . . . . 87

8.3 Jet $p_{T}$ spectra for various classes of $\mathrm{z}$ distribution of the primary vertex. 92

8.4 Unfolding Correction factors as a function of jet $p_{T}$ for $R=0.4$ jets as defined in Equation (8.9) for the four central rapidity bins along with systematic uncertainties on these factors due to uncertainties in the jet energy and angular resolution, and the MC spectrum shape. . . . . .

8.5 Unfolding Correction factors as a function of jet $p_{T}$ for $R=0.4$ jets as defined in Equation (8.9) for the three forward rapidity bins along with systematic uncertainties on these factors due to uncertainties in the jet energy and angular resolution, and the MC spectrum shape. .

8.6 Unfolding Correction factors as a function of jet $p_{T}$ for $R=0.6$ jets as defined in Equation (8.9) for the four central rapidity bins along with systematic uncertainties on these factors due to uncertainties in the jet energy and angular resolution, and the $\mathrm{MC}$ spectrum shape. . . . . .

8.7 Unfolding Correction factors as a function of jet $p_{T}$ for $R=0.6$ jets as defined in Equation (8.9) for the three forward rapidity bins along with systematic uncertainties on these factors due to uncertainties in the jet energy and angular resolution, and the MC spectrum shape. . 100

8.8 Probability of having $N_{\mathrm{PV}}$ primary vertices in a given collision event and in a particular $y-p_{T}$ bin: (a) the central region with $0.3<|\eta|<0.8$ and $20 \mathrm{GeV}<p_{T}<30 \mathrm{GeV}$, (b) the transition region $(2.8<|\eta| \leq 3.6$ and $60 \mathrm{GeV}<p_{T}<80 \mathrm{GeV}$, and (c) the forward region $(3.6<|\eta| \leq 4.5$ and $\left.110 \mathrm{GeV}<p_{T}<160 \mathrm{GeV}\right) . \ldots \ldots \ldots 101$

8.9 Systematic uncertainty of the inclusive jet cross section measurement due to JES for anti- $k_{T}$ jets with $R=0.4$ derived using the shape of the MC10 Pythia dijet sample along with the JES uncertainty. The JES uncertainty due to pile up is taken into account using a conservative estimate of the pile-up uncertainty and the $N_{\mathrm{PV}}$ distribution observed in data. 
8.10 Systematic uncertainty of cross section measurement due to JES for anti- $k_{T}$ jets with $R=0.6$ derived using the shape of the MC10 Pythia dijet sample along with the JES uncertainty. The JES uncertainty due to pile up is taken into account using a conservative estimate of the pile-up uncertainty and the $N_{\mathrm{PV}}$ distribution observed in data. . . .

8.11 Inclusive jet double-differential cross section as a function of jet $p_{T}$ in different regions of $|y|$ for jets identified using the anti- $k_{T}$ algorithm with $R=0.4$. For convenience, the cross sections are multiplied by the factors indicated in the legend. The data are compared to NLO pQCD calculations to which non-perturbative corrections have been applied. The error bars indicate the statistical uncertainty on the measurement, and the shaded band indicates the quadratic sum of the systematic uncertainties, dominated by the jet energy scale uncertainty. There is an additional overall uncertainty of $3 \%$ due to the luminosity measurement that is not shown. The theoretical uncertainty shown is the quadratic sum of uncertainties from the choice of renormalisation and factorisation scales, parton distribution functions, $\alpha_{s}\left(M_{Z}\right)$, and the modelling of non-perturbative effects, as described in the text. . . . . . . .

8.12 Inclusive jet double-differential cross section as a function of jet $p_{T}$ in different regions of $|y|$ for jets identified using the anti- $k_{T}$ algorithm with $R=0.6$. For convenience, the cross sections are multiplied by the factors indicated in the legend. The data are compared to NLO pQCD calculations to which non-perturbative corrections have been applied.The error bars indicate the statistical uncertainty on the measurement, and the shaded band indicates the quadratic sum of the systematic uncertainties, dominated by the jet energy scale uncertainty. There is an additional overall uncertainty of $3 \%$ due to the luminosity measurement that is not shown. The theoretical uncertainty is the quadratic sum of uncertainties from the choice of renormalisation and factorisation scales, parton distribution functions, $\alpha_{s}\left(M_{Z}\right)$, and the mode-ling of non-perturbative effects, as described in the text. 
8.13 Inclusive jet double-differential cross section as a function of jet $p_{T}$ in different regions of $|y|$ for jets identified using the anti- $k_{T}$ algorithm with $R=0.4$. The ratio of the data to the theoretical prediction is shown, and the total systematic uncertainties on the theory and measurement are indicated. The theoretical and experimental uncertainties are calculated as described in Figure 8.11. . . . . . . . . . . .

8.14 Inclusive jet double-differential cross section as a function of jet $p_{T}$ in different forward regions of $|y|$ for jets identified using the anti- $k_{T}$ algorithm with $R=0.4$. The ratio of the data to the theoretical prediction is shown, and the total systematic uncertainties on the theory and measurement are indicated. The theoretical and experimental uncertainties are calculated as described in Figure 8.11. . . . . . . . .

8.15 Inclusive jet double-differential cross section as a function of jet $p_{T}$ in different regions of $|y|$ for jets identified using the anti- $k_{T}$ algorithm with $R=0.6$. The ratio of the data to the theoretical prediction is shown, and the total systematic uncertainties on the theory and measurement are indicated. The theoretical and experimental uncertainties are calculated as described in Figure 8.12. . . . . . . . . . . . 111

8.16 Inclusive jet double-differential cross section as a function of jet $p_{T}$ in different forward regions of $|y|$ for jets identified using the anti- $k_{T}$ algorithm with $R=0.6$. The ratio of the data to the theoretical prediction is shown, and the total systematic uncertainties on the theory and measurement are indicated. The theoretical and experimental uncertainties are calculated as described in Figure 8.12. . . . . . . . . 112

8.17 Inclusive jet double-differential cross section as a function of jet $p_{T}$ in different central regions of $|y|$ for jets identified using the anti- $k_{T}$ algorithm with $R=0.4$. The theoretical error bands obtained by using different PDF sets (CTEQ 6.6, MSTW 2008, NNPDF 2.1, HERA 1.5) are shown. The data points and the error bands are normalized to the theoretical estimates obtained by using the CTEQ 6.6 PDF set. . . . 113 
8.18 Inclusive jet double-differential cross section as a function of jet $p_{T}$ in different forward regions of $|y|$ for jets identified using the anti- $k_{T}$ algorithm with $R=0.4$. The theoretical error bands obtained by using different PDF sets (CTEQ 6.6, MSTW 2008, NNPDF 2.1, HERA 1.5) are shown. The data points and the error bands are normalized to the theoretical estimates obtained by using the CTEQ 6.6 PDF set. . . .

8.19 Inclusive jet double-differential cross section as a function of jet $p_{T}$ in different central regions of $|y|$ for jets identified using the anti- $k_{T}$ algorithm with $R=0.6$. The theoretical error bands obtained by using different PDF sets (CTEQ 6.6, MSTW 2008, NNPDF 2.1, HERA 1.5) are shown. The data points and the error bands are normalized to the theoretical estimates obtained by using the CTEQ 6.6 PDF set. . . .

8.20 Inclusive jet double-differential cross section as a function of jet $p_{T}$ in different forward regions of $|y|$ for jets identified using the anti- $k_{T}$ algorithm with $R=0.6$. The theoretical error bands obtained by using different PDF sets (CTEQ 6.6, MSTW 2008, NNPDF 2.1, HERA 1.5) are shown. The data points and the error bands are normalized to the theoretical estimates obtained by using the CTEQ 6.6 PDF set. . . . 116

8.21 Inclusive jet double-differential cross section as a function of jet $p_{T}$ in different regions of $|y|$ for jets identified using the anti- $k_{T}$ algorithm with $R=0.4$. The ratio of the Powheg predictions showered by Pythia and Herwig to the NLO predictions corrected for the non-perturbative effects is shown. The ratio shows only the statistical uncertainty on the Powheg prediction, and can be compared to the ratio of the data to the NLO pQCD prediction (corrected for non-perturbative effects). The total systematic uncertainties on the theory and measurement are indicated. The NLO pQCD prediction and the Powheg ME calculations use the MSTW PDF set. . . . . . . . . . . . . . . . . . . . . . . 117 
8.22 Inclusive jet double-differential cross section as a function of jet $p_{T}$ in different forward regions of $|y|$ for jets identified using the anti- $k_{T}$ algorithm with $R=0.4$. The ratio of the Powheg predictions showered by Pythia and Herwig to the NLO predictions corrected for the non-perturbative effects is shown. The ratio shows only the statistical uncertainty on the Powheg prediction, and can be compared to the ratio of the data to the NLO pQCD prediction (corrected for nonperturbative effects). The total systematic uncertainties on the theory and measurement are indicated. The NLO pQCD prediction and the Powheg ME calculations use the MSTW PDF set. . . . . . . . . . . 118

8.23 Inclusive jet double-differential cross section as a function of jet $p_{T}$ in different regions of $|y|$ for jets identified using the anti- $k_{T}$ algorithm with $R=0.6$. The ratio of the Powheg predictions showered by Pythia and Herwig to the NLO predictions corrected for the non-perturbative effects is shown. The ratio shows only the statistical uncertainty on the Powheg prediction, and can be compared to the ratio of the data to the NLO pQCD prediction (corrected for non-perturbative effects). The total systematic uncertainties on the theory and measurement are indicated. The NLO pQCD prediction and the Powheg ME calculations use the MSTW PDF set. . . . . . . . . . . . . . . . . .

8.24 Inclusive jet double-differential cross section as a function of jet $p_{T}$ in different forward regions of $|y|$ for jets identified using the anti- $k_{T}$ algorithm with $R=0.6$. The ratio of the Powheg predictions showered by Pythia and Herwig to the NLO predictions corrected for the non-perturbative effects is shown. The ratio shows only the statistical uncertainty on the Powheg prediction, and can be compared to the ratio of the data to the NLO $\mathrm{pQCD}$ prediction (corrected for nonperturbative effects). The total systematic uncertainties on the theory and measurement are indicated. The NLO pQCD prediction and the Powheg ME calculations use the MSTW PDF set. . . . . . . . . . 120 
8.25 Dijet double-differential cross section as a function of dijet mass, binned in the maximum rapidity of the two leading jets $|y|_{\max }$. The results are shown for jets identified using the anti- $k_{T}$ algorithm with $R=$ 0.4. The data are compared to NLO pQCD calculations to which non-perturbative corrections have been applied. The systematic and theoretical uncertainties are calculated as described in Figure 8.11. .

8.26 Dijet double-differential cross section as a function of dijet mass, binned in the maximum rapidity of the two leading jets $|y|_{\max }$. The results are shown for jets identified using the anti- $k_{T}$ algorithm with $R=$ 0.6. The data are compared to NLO pQCD calculations to which non-perturbative corrections have been applied. The systematic and theoretical uncertainties are calculated as described in Figure 8.12. .

8.27 Dijet double-differential cross section as a function of dijet mass, binned in the maximum rapidity of the two leading jets $|y|_{\max }$. The results are shown for jets identified using the anti- $k_{T}$ algorithm with $R=$ 0.4. The data are compared to NLO pQCD calculations to which non-perturbative corrections have been applied. The systematic and theoretical uncertainties are calculated as described in Figure 8.11. .

8.28 Dijet double-differential cross section as a function of dijet mass, binned in the maximum rapidity of the two leading jets $|y|_{\max }$. The results are shown for jets identified using the anti- $k_{T}$ algorithm with $R=$ 0.6. The data are compared to NLO pQCD calculations to which non-perturbative corrections have been applied. The systematic and theoretical uncertainties are calculated as described in Figure 8.12. .

8.29 Dijet double-differential cross section as a function of dijet mass, binned in the maximum rapidity of the two leading jets $|y|_{\max }$. The results are shown for jets identified using the anti- $k_{T}$ algorithm with $R=$ 0.4. The data, as well as NLO pQCD predictions corrected for nonperturbative effects and using different PDF sets, are shown normalized to the theoretical prediction using the CTEQ 6.6 central value. No uncertainties are shown. For clarity, this diagram should be viewed in colour. 
8.30 Dijet double-differential cross section as a function of dijet mass, binned in the maximum rapidity of the two leading jets $|y|_{\max }$. The results are shown for jets identified using the anti- $k_{T}$ algorithm with $R=$ 0.6. The data, as well as NLO pQCD predictions corrected for nonperturbative effects and using different PDF sets, are shown normalized to the theoretical prediction using the CTEQ 6.6 central value. No uncertainties are shown. For clarity, this diagram should be viewed in colour.

8.31 Dijet double-differential cross section as a function of dijet mass, binned in the maximum rapidity of the two leading jets $|y|_{\max }$. The results are shown for jets identified using the anti- $k_{T}$ algorithm with $R=0.4$. The data are compared to Powheg, a Monte Carlo with a NLO matrix element interfaced to a matched parton shower, hadronization, and underlying event modelled by Pythia. The experimental uncertainties are calculated as described in Figure 8.11.

8.32 Dijet double-differential cross section as a function of dijet mass, binned in the maximum rapidity of the two leading jets $|y|_{\max }$. The results are shown for jets identified using the anti- $k_{T}$ algorithm with $R=0.4$. The data are compared to Powheg, a Monte Carlo with a NLO matrix element interfaced to a matched parton shower, hadronization, and underlying event modelled by Herwig. The experimental uncertainties are calculated as described in Figure 8.11. . . . . . . . .

8.33 Dijet double-differential cross section as a function of dijet mass, binned in the maximum rapidity of the two leading jets $|y|_{\max }$. The results are shown for jets identified using the anti- $k_{T}$ algorithm with $R=0.6$. The data are compared to Powheg, a Monte Carlo with a NLO matrix element interfaced to a matched parton shower, hadronization, and underlying event modelled by Pythia. The experimental uncertainties are calculated as described in Figure $8.12 \ldots \ldots \ldots \ldots$ 
8.34 Dijet double-differential cross section as a function of dijet mass, binned in the maximum rapidity of the two leading jets $|y|_{\max }$. The results are shown for jets identified using the anti- $k_{T}$ algorithm with $R=0.6$. The data are compared to Powheg, a Monte Carlo with a NLO matrix element interfaced to a matched parton shower, hadronization, and underlying event modelled by Herwig. The experimental uncertainties are calculated as described in Figure 8.12 . . . . . . . . . . . . 132 


\section{Chapter 1}

\section{Introduction}

In the spring of 2010 the Large Hadron Collider (LHC) began colliding protons at an unprecedented center-of-mass energy of $7 \mathrm{TeV}$. An electronvolt $(\mathrm{eV})$ is a unit of energy defined as the amount of kinetic energy gained by an electron accelerated through a potential difference of 1 Volt. Hence $1 \mathrm{TeV}$ is one Tera-electronvolt = $10^{12} \mathrm{eV}$. The first collision run lasted roughly eight months and produced about $37 \mathrm{pb}^{-1}$ of data which corresponds to a total of about $3.7 \times 10^{12}$ proton-proton collisions. This is a small amount of data in comparison to the expected amount needed for physics discovery which is on the order of several thousand $\mathrm{pb}^{-1}$. However, the early data serve as key samples for detector commissioning, allowing for in-situ detector calibration and performance assessment. The data also allow for a first look at subatomic physics at the $\mathrm{TeV}$ scale, in an unexplored energy regime.

This thesis is a description of two distinct but related analyses of the data. The first involves commissioning of the ATLAS detector [4], which provides an assessment of its calorimetry system. The second is a physics analysis that focuses on jet production. 
The thesis is organised as follows. The remainder of Chapter 1 describes the general physics goals of the LHC experiments and high energy physics in general. Chapter 2 provides the theoretical background of the strong interaction and the production of jets. Both the commissioning and physics analyses of the thesis are primarily based on observation of jets, so a general overview of their nature is warranted. Chapter 3 gives an overview of the experimental setup, describing both the LHC and the ATLAS detector. Chapter 4 discusses some general aspects of calorimetry and the observation of jets in high energy physics experiments. Chapter 5 explains how jets are identified in the ATLAS calorimeters. Chapter 6 contains the commissioning analysis; an intercalibration of the ATLAS calorimeters using jets from collision data. Chapter 8 is the physics analysis, presenting results of the cross section measurements for inclusive jet and dijet production, followed by a summary in Chapter 9 .

\subsection{Physics Motivation}

\subsubsection{Standard Model}

High energy physics is the study of elementary particles and their interactions. It is a realm of physics that deals with the smallest scales of nature accessible by today's experiments. The physical world at these scales is described by the Standard Model (SM) of particle physics [5]. The two basic ingredients of the SM are elementary fermions and gauge bosons. Elementary fermions are the building blocks of matter, as we know it, and are further divided into quarks and leptons. Six types of quarks and equally as many leptons are known to exist. They are divided into three generations classifying them according to their mass and flavour. Each generation houses two 
quarks and two leptons.

Fermions interact with one another by exchanging gauge bosons, of which there are different types, giving rise to the four known interactions, listed in order of increasing strength: gravitational, weak, electromagnetic (EM), and the strong interaction. The SM, however, does not include gravity, as there is no experimentally verified and universally accepted quantum theory of gravity. Figure 1.1 displays all the fundamental particles present in the theory, including the Higgs boson, discussed below.

Figure 1.1: The elementary particles of the Standard Model. Fermilab National Accelerator Laboratory (c). http://www.fnal.gov/pub/presspass/images/LighterHiggs-images.html.

\section{Quarks}

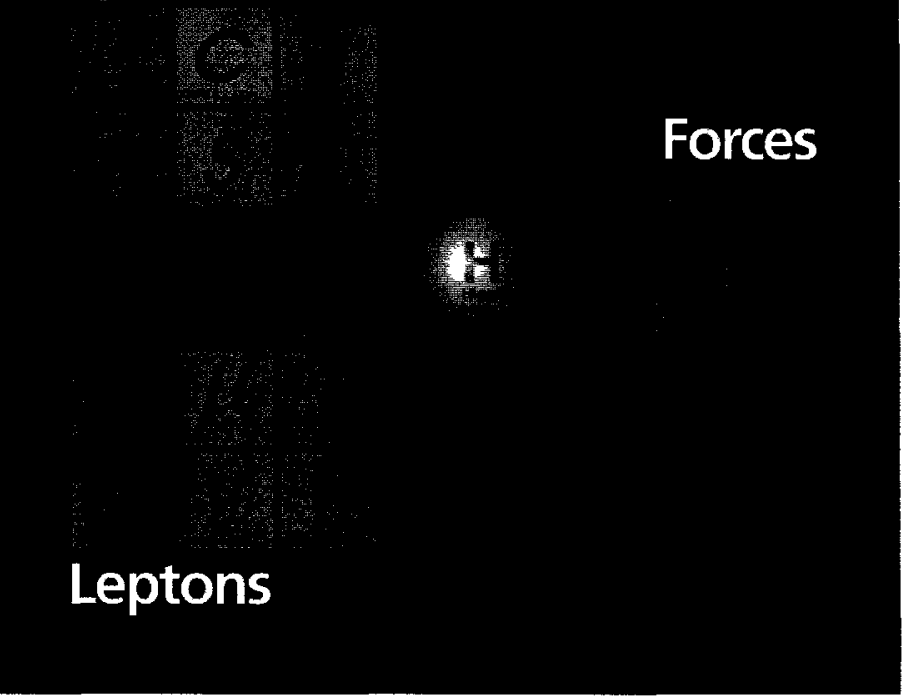

Based on the principles of quantum mechanics, the SM describes the weak, electromagnetic, and strong interactions. It has been thoroughly tested and verified experimentally to a great degree of precision. The weak interaction is mediated by massive $\mathrm{W}$ and $\mathrm{Z}$ bosons, and all known matter (quarks and leptons) participate in this interaction. The electromagnetic interaction is mediated by massless photons, 
and only electrically charged particles interact electromagnetically. The strong interaction, mediated by massless gluons, distinguishes quarks from leptons; quarks interact via the strong force while leptons do not. Just as photons couple to electrical charge, gluons couple to colour charge. Furthermore, gluons themselves carry colour charge and hence interact with one another. This self-interaction gives rise to some interesting properties of the strong interaction. This is discussed further in the next Chapter.

In order for the SM to be a complete and self-consistent theory incorporating massive particles, it requires the existence of a particle known as the Higgs boson. The Higgs can be thought of as a field permeating the Universe and coupling to certain particles, thereby giving them mass; the stronger the coupling, the higher the mass. Particles like the photon and gluon do not couple to the Higgs field and hence remain massless.

Many theoretical parameters of the SM are loosely constrained, particularly those of the theory of the strong interaction. The Large Hadron Collider (LHC) will allow for stringent testing of the SM in a new energy regime, providing more precise measurements of the key theoretical parameters. Although the SM has been very successful with theoretical predictions to date, the Higgs boson itself has never been observed and its existence has yet to be verified. This is a major challenge of today's experiments and it is one of the primary goals of the LHC.

\subsubsection{Beyond the Standard Model}

The SM has no theoretical framework describing gravity, hence it is an incomplete description of physical reality. The holy grail of physics today is to find a theory that 
unifies all the fundamental forces of nature into one theory. There exists a well known and successful theory of gravity, Albert Einstein's General Relativity (GR). However, GR describes gravity at the macro level, or large scales; it is based on classical physics, which differs greatly from quantum physics. In order to contrive a theory that unifies gravity with the other interactions, a quantum mechanical description of gravity is required. Indeed, there are theories that have made great advances in this quest and predicted new phenomena which may be testable in high energy experiments. One such phenomenon is known as Supersymmetry (SUSY), which states that each elementary fermion has a supersymmetric boson partner, and vice versa. In an unbroken SUSY theory, the SUSY partners are identical in all aspects, including mass, differing only in spin by half a unit. No SUSY particle has ever been observed, implying that if SUSY exists, it is a broken symmetry, i.e. the hypothetical and undiscovered SUSY particles are much heavier than their partners. With the energy reach at the LHC, the discovery of SUSY particles is a possibility.

Gravity has helped in discovering yet another mystery in the Universe. Based on our current understanding of gravity, careful investigation of the cosmos reveals that there isn't enough ordinary matter to hold galaxies and galaxy-clusters in place. The observed force of gravity is too strong for the amount of ordinary matter. One possible explanations for this is that we simply do not understand gravity as well as we think we do. Another explanation may be that there is some other force at play. However, the current best and most widely accepted explanation for the observed stronger force of gravity is due to extra mass. This mass is postulated to be from an unknown type of matter called dark matter which is invisible as it does not interact with photons, hence the name. This is another area of potential discovery at high 
energy experiments, as it may be possible to produce dark matter particles in high energy collisions.

It was mentioned in the previous section that the SM describes nature at the smallest scales. But one can always ask the question "how small does small get?". The reach of the SM is down to, on the order of, $10^{-18} \mathrm{~m}$, corresponding to an energy of roughly $200 \mathrm{GeV}$. What happens as we go further, down the realm of small, and higher in energy? Particle Physicists have no definite answer to this question, but many theories beyond the SM have been developed to address this question. With a design center-of-mass energy of $14 \mathrm{TeV}$, corresponding to a length resolution of $10^{-20}$ $\mathrm{m}$, the LHC is able to probe new grounds of physics and address this compelling question experimentally. 


\section{Chapter 2}

\section{Theoretical Background: Jets and QCD}

\subsection{Overview of the Strong Interaction}

Quantum Chromodynamics (QCD) is the theory that describes the strong interaction. The participants are quarks and gluons, collectively known as partons. Quarks are spin $1 / 2$ elementary fermions and they interact with one another by exchanging gluons, which are spin 1 gauge bosons. In this sense QCD is quite similar to the quantum theory of electromagnetic interactions, quantum electrodynamics (QED). There are, however, three very important fundamental differences. First, the coupling between quarks and gluons is significantly stronger than the coupling between charged elementary fermions and photons, hence the name 'strong'. More precisely, the strong coupling is significantly larger than the EM coupling at distances that are probed in today's experiments. The coupling strengths are in fact not constant, but rather vary as functions of the distance, or the momentum exchange, between 
the fermions. It is believed that at extremely high energies, or small distances, the strengths of the all interactions become equivalent. This is thought to be true for all interactions of nature, including gravity.

Second, and of more profound importance, unlike photons which are electrically neutral, gluons carry colour charge and hence couple to one another. This gives rise to two important properties of the strong interaction: asymptotic freedom and colour confinement. These are actually not two independent properties, but are really two extremes of the same concept. Asymptotic freedom is the phenomenon whereby the coupling strength of the strong interaction decreases at smaller distances or higher energies. The closer two quarks get to one another, the weaker the interaction between them. Conversely, the larger the distance between the quarks, the stronger the interaction. This increase in strength is the source of colour confinement which is the property that forbids the existence of a free colour-charged state. This means that an isolated colour-charged particle can never be observed. Particles that interact via the strong force that are observed in nature are called hadrons. They are composed of either two quarks (quark anti-quark pairs known as mesons) or three quarks (baryons), forming a colour singlet state such that the net colour charge is zero. Technically, since hadrons are colour neutral, they do not interact with one another by exchanging gluons. Their interaction, the nuclear force, is a remnant of the strong force, occurring via meson exchange.

Lastly, in electromagnetic interactions, there exists only one type of charge which can be positive or negative. The strong interaction, on the other hand, embodies three types of colour charge, referred to as red, blue, and green, which also can be positive or negative. The negative charges are known as anti-colours. The pairing of 
a colour and its anti-colour as well as the combination of three distinct colour charges, of the same polarity, is colour neutral. This is how mesons and baryons remain colour singlets.

\subsection{Jets}

Colour confinement leads to a very important aspect, especially in experimental particle physics. At separation distances between two partons beyond about $0.1 \mathrm{fm}$ [6] the strong force increases rapidly, which means that in order to separate the two, an infinite amount of energy would be required. This is why isolated partons are never observed in nature. However, this does not imply that hadrons can not be destroyed. If a hadron is bombarded with sufficient impact, i.e. in a high energy collision, the internal partons may begin to separate, causing the energy in the force field between them to grow. Generally the partons are not pulled back together. Instead, the excess energy is given off by creating new quark-antiquark pairs. Moreover, as the partons are pulled apart, they become moving colour charges and radiate gluons that create more quark-antiquark pairs. This process is known as fragmentation. The newly created colour particles combine to form colour neutral hadrons, through a process called hadronization. The end result is a collinear beam of particles, typically dozens at the LHC, with total momentum closely resemblant to the original parton. This spray of particles is called a jet, and it is the most copious product and key observable in high energy proton-proton collisions.

From an experimental perspective, there are generally three levels of a jet: parton, particle, and reconstruction level. The parton jet is conceptual and never observed, corresponding to the single parton from which the jet evolves. The particle level jet, 
commonly referred to as the truth jet, is the above mentioned stream of particles that can be observed in nature. The reconstruction level jet, also referred to as the calorimeter level jet, corresponds to how the particle level jet is observed experimentally. This is usually an energy cluster translated into an electronic signal, left in the detector (calorimeter), from the traversing particle jet. An important and rather challenging objective of today's experiments is to have a perfect match between truth and calorimeter level jets, i.e. to have the same kinematical properties such as energy, momentum, and position. Chapter 5 gives details about how this challenge is addressed in the ATLAS detector.

\subsection{Parton Distribution Functions (PDFs)}

As hadrons are composite particles, their momentum is carried by their constituent partons. A parton distribution function (PDF) is a probability density for finding a parton with a certain longitudinal momentum fraction $x$ of the hadron. Consider for example a neutron, which is known to be composed of two down quarks and one up quark. If the masses of the up and down quarks are assumed to be the same, then it may be natural to believe that each quark carries on average $1 / 3$ of the neutron's momentum. This is not the case, however, as the gluons that bind the quarks together, also carry a significant momentum fraction, as much as $55 \%$ [7]. Furthermore, a small fraction of the momentum may be carried by quarks other than the up and down valence quarks. Quantum fluctuations allow gluons to produce quark anti-quark pairs of any flavour, annihilating back into a gluon. These momentarily present quarks are referred to as sea quarks. A PDF contains information about the momentum distribution of the partons, both valance and sea partons. The PDFs are 
not theoretically calculable; they can only be extracted by fitting experimental data. Many different sets of PDFs exist today, obtained from different experiments and different groups. Some of these will be mentioned and used for the jet cross section analysis presented in Chapter 8

\subsection{Hard Scatter and the Underlying Event}

In a typical high energy p-p collision event, such as one at the LHC, the collision is generally not the result of the two protons, as whole objects, interacting with one another. Instead, the collision results from the interaction of an individual parton from one proton with a parton from the other proton. At lowest order, this interaction is a simple $2 \rightarrow 2$ process; two partons come in, two partons, which turn in to two jets, go out. This $2 \rightarrow 2$ process is referred to as the hard scatter of the event.

The partons that do not participate in the hard scatter are temporarily left behind as isolated colour charges; they hence immediately hadronize and form jets. This activity away from the hard scatter is known as the underlying event. 


\section{Chapter 3}

\section{Experimental Setup}

\subsection{CERN}

The European Organization for Nuclear Research (CERN), based in Geneva, Switzerland, was founded in 1952 with a mandate of conducting world-class fundamental physics research. The name CERN stems from the French acronym "Conseil Européen pour la Recherche Nucléaire". Over the years many important discoveries and inventions have arisen from CERN, including the W and Z bosons in 1983 and the World Wide Web (www) in 1989. Of course, fundamental physics research has evolved greatly and the term 'nuclear' is outdated. Today, the primary focus of CERN is well beyond nuclear physics; it is to study the fundamental constituents of matter and the forces acting between them. 


\subsection{LHC}

The Large Hadron Collider (LHC) [8], located at CERN, is the world's largest and most powerful tool for probing the structure of matter. It is a $26.7 \mathrm{~km}$ long superconducting hadron accelerator, located $100 \mathrm{~m}$ below the earth's surface. It consists of two rings that circulate beams of protons (or lead ions) in opposite directions and bring them into collisions at four interaction points of the LHC, which correspond to the four main detectors (ALICE, ATLAS, CMS, and LHCb) [8]. The $7.2 \mathrm{~cm}$ wide beam pipe, where the hadrons travel, is maintained at extremely low pressures of $10^{-13} \mathrm{~atm}$.

The design center of mass collision energy for protons is $14 \mathrm{TeV}$ with a peak machine luminosity of $10^{34} \mathrm{~cm}^{-2} \mathrm{~s}^{-1}$. The corresponding numbers for lead ion collisions are $5.5 \mathrm{TeV}$ per nucleon and $10^{27} \mathrm{~cm}^{-2} \mathrm{~s}^{-1}$, respectively. The ALICE experiment is optimised to study the lead ion collisions. Its key motivation is to learn about the strong interaction at extremely high energy densities and temperatures, akin to the conditions in the early moments of the universe. For a much more detailed discussion of ALICE and its goals, see reference [8]. Since this thesis is based on proton-proton $(\mathrm{p}-\mathrm{p})$ collisions, the remainder of the discussion will be in terms of protons rather than lead ions.

Before the protons are brought into collision, they undergo a 5-stage acceleration process that brings them up to the final collision energy. Starting from compressed hydrogen gas cylinders, hydrogen atoms are fed at a precise rate into the source chamber of a linear accelerator (LINAC). They are heated to the point of ionization and the protons are separated from their electrons by applying an external electric field. The LINAC is stage 1 of the acceleration process and it brings the protons to a 
speed of about $1 / 3$ the speed of light $(0.33 \mathrm{c})$. Next the proton beam is split into four, and each enters a circular ring called the Booster, which is actually four $157 \mathrm{~m}$ long rings placed on top of each other, one for each beam. At this point linear acceleration is impractical and the protons are accelerated repeatedly each time they circulate the booster ring. Using pulsed electric fields to accelerate, and electromagnets to keep the motion circular, the Booster (stage 2) accelerates the protons to speeds of $0.916 \mathrm{c}$. In stage 3, the four beams are recombined into one and it is transferred over to the Proton Synchrotron (PS), which is simply a much larger circular accelerator with a circumference of $628 \mathrm{~m}$. In the same manner, the protons are accelerated to $0.999 \mathrm{c}$, corresponding to an energy of $25 \mathrm{GeV}$. Stage 4 takes the protons to an even larger circular accelerator, the Super Proton Synchrotron (SPS), with a circumference of 7 $\mathrm{km}$. Here the protons are brought to $450 \mathrm{GeV}$ before being sent to the final stage (stage 5) which is the LHC itself. The LHC takes the $450 \mathrm{GeV}$ protons and repeatedly circulates them until they reach the final energy of $7 \mathrm{TeV}$. A magnetic field strength of 8.33 Tesla $(\mathrm{T})$ is required to the keep the beam in circular motion in the LHC. These large field strengths are generated by powerful superconducting electromagnets operating on a current of $11.85 \mathrm{kA}$. To remain superconducting, the magnets are kept at an extremely low temperature of $2 \mathrm{~K}$.

\subsection{The ATLAS Detector}

ATLAS (A Toroidal Lhc ApparatuS) is a general purpose detector [4] of the LHC. Its primary goal is to find and study the hypothesized Higgs boson. Additional goals include precision measurements of the SM parameters, and searches for new physics beyond the SM, such as SUSY, extra dimensions, and dark matter. Its construction 
has been guided by these goals so that the physics discovery potential is optimized. Figure 3.1 shows a schematic of the ATLAS detector with some of its characteristics. It is a multipurpose machine, built of many layers, each with a specific and important task in detecting the collision products and reconstructing the physics event. The Inner Detector (ID) is the central core and it provides tracking of charged particles, which allows for precise localisation of the interaction point, commonly referred to as the primary vertex. Inside the ID is the Central Solenoid (CS) that provides a $2 \mathrm{~T}$ magnetic field that bends the particle tracks thus enabling precise momentum measurements. The next layer is the electromagnetic (EM) calorimetry system, which provides precise energy and position measurements of electrons and photons. Hadronic particles will generally penetrate through the EM calorimeter and be contained in the next layer, the hadronic calorimetry system, which provides jet and missing energy measurements. Finally, muons will penetrate the hadronic calorimeters and will leave a track in the muon system, which precisely measures their momenta. Subsequent sections will give more detailed characteristics of each sub-detector.

\subsubsection{Coordinate Systems and Common Variables}

ATLAS makes use of all three major coordinate systems: cartesian, cylindrical, and spherical. The origin is the detector center. The beam line defines the $\mathrm{z}$ axis, and the transverse plane is defined with respect to the beam line. The positive $\mathrm{x}$ axis points towards the center of the LHC ring, and the y axis points upward, perpendicular to the earth's surface. The radial coordinate is $r=\sqrt{x^{2}+y^{2}+z^{2}}$ and the analogous coordinate in the $\mathrm{x}-\mathrm{y}$ plane is $\rho=\sqrt{\mathrm{x}^{2}+\mathrm{y}^{2}}$. The polar angle $\theta$ is measured from the 
Figure 3.1: Schematic of the ATLAS Detector. ATLAS Experiment (c) 2011 CERN. http://www.atlas.ch/photos/full-detector.html.

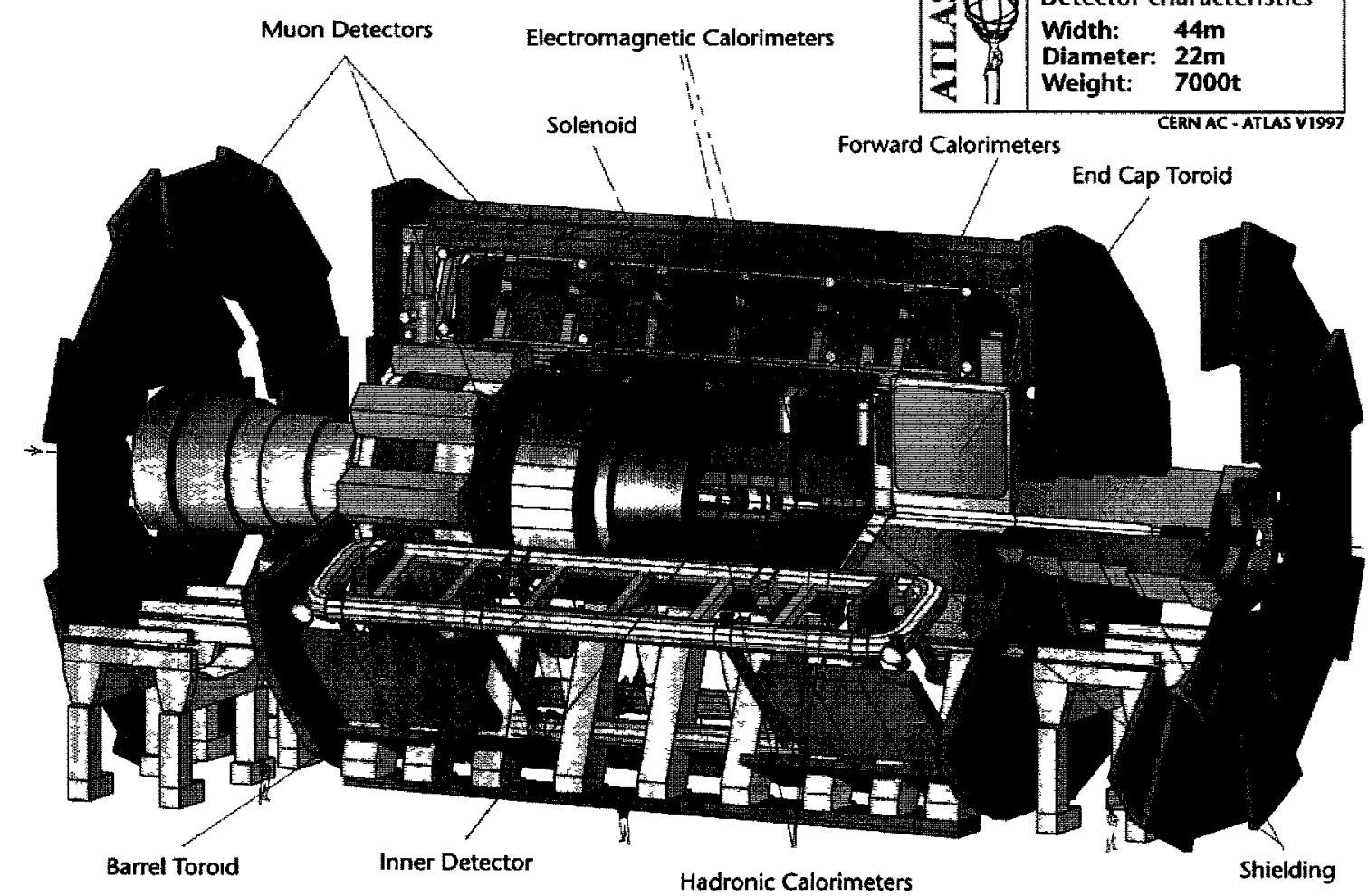

$\mathrm{z}$ axis. The pseudorapidity is defined as

$$
\eta \equiv-\ln [\tan (\theta / 2)]
$$

and it is used instead of $\theta$. The azimuthal angle $\phi$ is measured around the beam axis from $-\pi$ to $\pi$. A commonly used separation variable between two objects, i.e. particles or jets, is $\Delta R \equiv \sqrt{\Delta \eta^{2}+\Delta \phi^{2}}$, where $\Delta \eta \equiv \eta_{2}-\eta_{1}$, and $\Delta \phi \equiv \min \left(\left|\phi_{2}-\phi_{1}\right|, 2 \pi-\right.$ $\left.\left|\phi_{2}-\phi_{1}\right|\right)$ so that $0 \leq \Delta \phi<\pi$. Two very common variables are the transverse energy $E_{T}$ and transverse momentum $p_{T}$, which are respectively the components of energy 
and momentum perpendicular to the beam line.

\subsubsection{Inner Detector and Tracking}

The Inner Detector (ID) forms the core of ATLAS. It is cylindrical in shape, surrounding the beam line, and has a length of $6.2 \mathrm{~m}$ and a radius of $1.08 \mathrm{~m}$. Surrounded by a Central Solenoid (CS) that provides a nominal magnetic field of $2 \mathrm{~T}$, the ID provides tracking of charged particles up to $|\eta|<2.5$. It is segmented into three sub-detectors, extending away from the beam line. These are the Pixel Detector, Semiconductor Tracker, and Transition Radiation Tracker, which provide tracking information with decreasing precision as a function of radial distance from the beam line. Figure 3.2 shows the inner detector.

The Pixel Detector (PD) is the module closest to the beam line and hence provides the most precise tracking due to its high granularity. In the central region it consists of three barrels, surrounding the beam pipe, at radii of approximately $5 \mathrm{~cm}, 9 \mathrm{~cm}$, and $12 \mathrm{~cm}$, while in the end-cap regions, the pixels are mounted on five disks perpendicular to the beam line. The pixels employ semiconductor silicon technology and provide an electrical signal via charge collection that arises from electron-hole pair creation. The pixels are segmented in $\rho \phi-\mathrm{z}$ space and afford a resolution of $12 \mu \mathrm{m}$ in $\rho \phi$ and $66 \mu \mathrm{m}$ in $\mathrm{z}$.

The next layer, Semiconductor Tracker, is a simple extension of the PD, with slightly coarser granularity. It uses silicon microstrips (SCT) and provides a spatial resolution of $16 \mu \mathrm{m}$ by $580 \mu \mathrm{m}$ in $\rho \phi$-z space. Eight layers of SCT cells surround the $\mathrm{PD}$, providing eight hits per track.

The last layer of the ID is the Transition Radiation Tracker (TRT), with approxi- 
Figure 3.2: Schematic of the ATLAS Inner Detector. ATLAS Experiment (c) 2011 CERN. http://www.atlas.ch/photos/inner-detector.html.

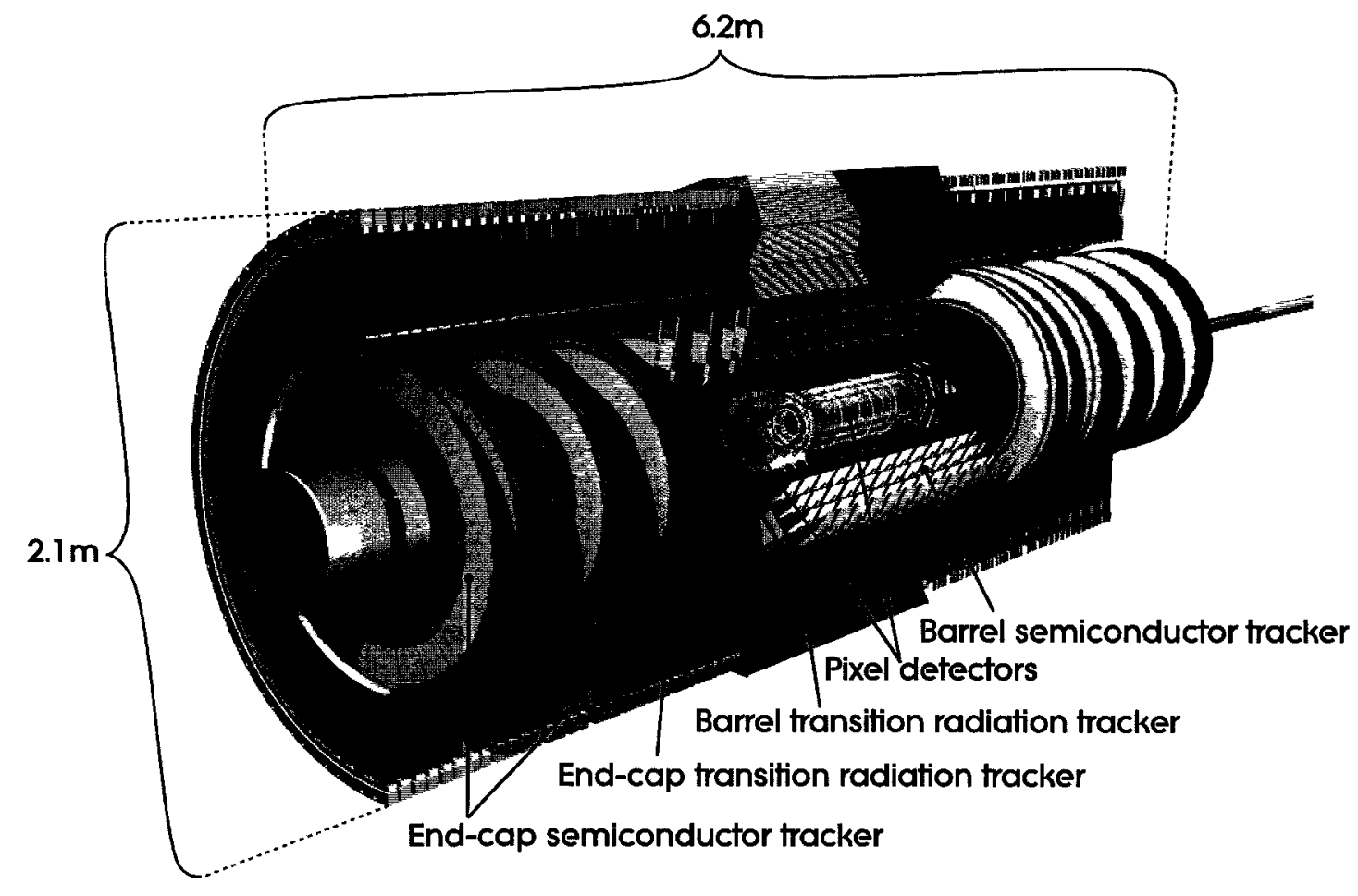

mate inner and outer radii of $55 \mathrm{~cm}$ and $1 \mathrm{~m}$, respectively. It employs roughly 370000 cylindrical drift tubes (straws) of $4 \mathrm{~mm}$ diameter, each equipped with a $30 \mu \mathrm{m}$ diameter gold-plated Tungsten-Rhenium (W-Re) anode wire. The straws have conductive coating and are kept at high voltage with negative polarity, and hence act as cathodes. The spatial resolution obtained by each straw is $170 \mu \mathrm{m}$. The straws are filled with a gas mixture of mostly xenon, that allows the detector to be sensitive to transition radiation photons emitted by traversing electrons. This feature provides good electron identification, i.e. separation of electrons and pions. Each track traverses about 36 straws, contributing significantly to the momentum measurement, because 
the lower precision per point compared to the silicon detectors is compensated by the larger number of measurements. The relative precision of the measurements in the three sub-detectors is well matched, so that no single measurement dominates. This implies a robust overall performance.

\subsubsection{Calorimeters}

Past the tracking detector lies the calorimetry system. As the name implies, calorimeters measure the energy of traversing particles. The ATLAS calorimeters are designed to provide very good performance in terms of energy and position measurements in order to achieve the desired physics goals mentioned at the beginning of Section 3.3.

There are two fundamental parts to the calorimetry system: electromagnetic (EM) and hadronic. The EM calorimeters will contain virtually all photons and electrons, and hence accurately measure their energy. Hadrons penetrate deeper than electrons due to their heavier mass, so additional material is required to fully stop them and properly measure their energy. In ATLAS, sampling calorimeters are used exclusively, with various active and passive materials. The passive medium is generally a dense heavy metal that can absorb much of the particles' energy and initiate a shower, while the active medium produces the ionisation charge or scintillating photons that can be collected and converted into an electronic signal. Thus the passive material ensures that particles are contained in the calorimeter, while the active sections ensures the sampling of their energy is performed. Figure 3.3 shows a schematic of the ATLAS calorimetry system.

The calorimeters are divided in regions of pseudorapidity, each region having both an EM and a hadronic calorimeter. The central region hosts the Barrel Calorimeter. 
Figure 3.3: Schematic of the ATLAS calorimeters. ATLAS Experiment (C) 2011 CERN. http://www.atlas.ch/photos/calorimeters.html.

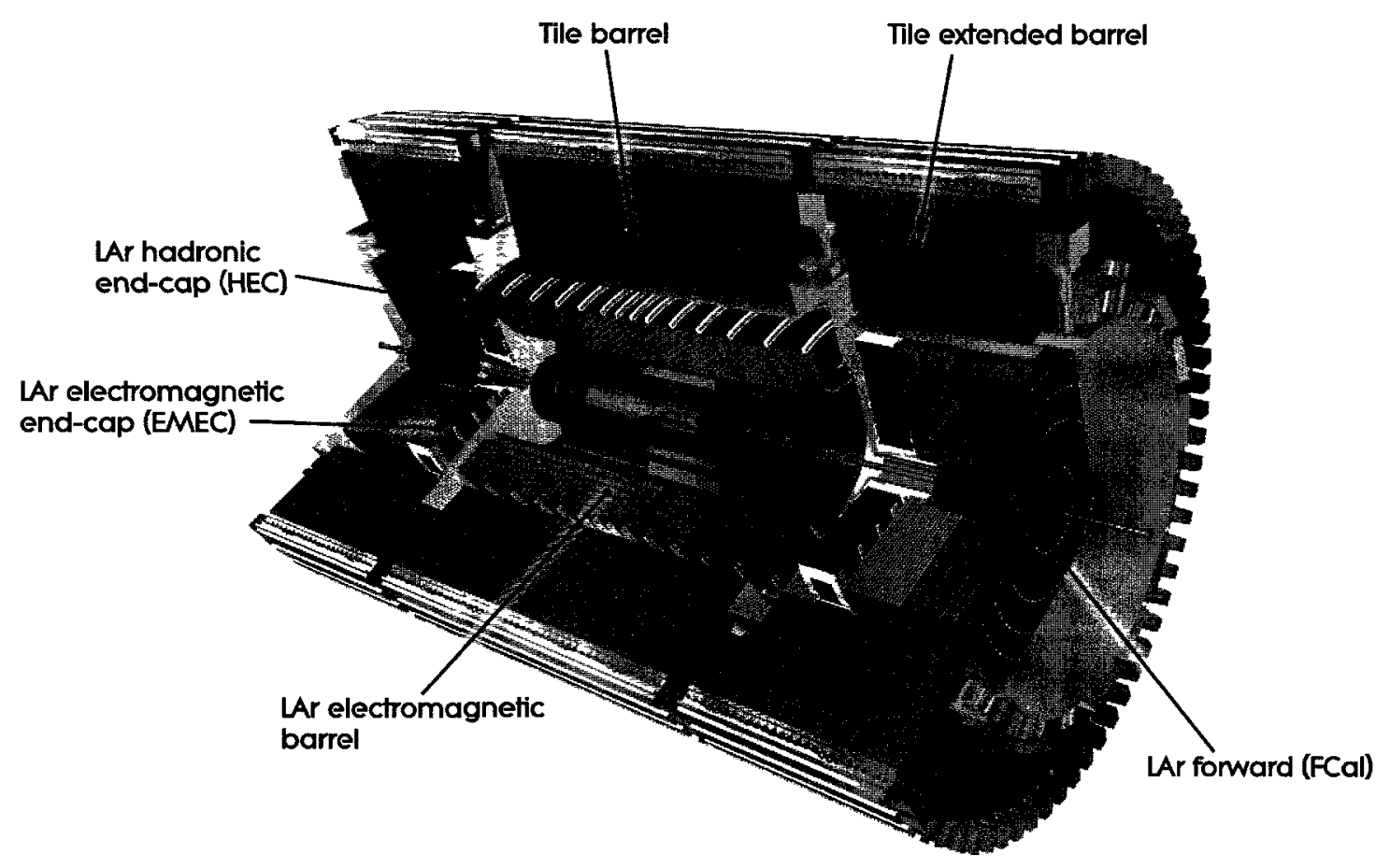

The EM component covers the range $|\eta|<1.475$, and uses liquid argon (LAr) as the active medium, producing ionisation charge for signal collection, and lead plates as the absorbers (passive medium). It has a projective 'accordion' geometry such that the towers are made to point to the detector center. It employs very high granularity $(\Delta \eta \times \Delta \phi)$ which varies with the calorimeter depth; $0.003 \times 0.1$ in the first sampling layer, $0.025 \times 0.025$ in the second, and $0.05 \times 0.025$ in the third. In the region $|\eta|<1.8$, a presampler detector is used to correct for the energy lost by electrons and photons in the material before the calorimeter (ID, CS, support structures). The total thickness of the EM barrel calorimeter is about 24 radiation lengths $\left(\mathrm{X}_{0}\right)$, where 
$\mathrm{X}_{0}$ is defined as the average thickness of a material required to decrease the energy of a high energy electron by a factor of $1 / e[9]$.

The hadronic barrel calorimeter, covering $|\eta|<1.7$, uses scintillating plastic as the active material and steel plates as the absorber. The scintillating photons are collected by photomultiplier tubes for signal readout. It is further divided into a central barrel covering $|\eta|<1.0$, and two extended barrels, one on each side, covering $0.8<|\eta|<1.7$. A more coarse granularity of $0.1 \times 0.1$ is sufficient for jet and missing $E_{T}$ measurements. The total thickness at $\eta=0$ is 9.7 nuclear absorption lengths $(\lambda)$, where $\lambda$ is the mean distance travelled by a hadronic particle before undergoing a nuclear interaction [10]. The composite barrel calorimeter is divided right in the middle at $\eta=0$. The material used to join the two halves, i.e. bolts, screws, etc., slightly hinders the calorimeter performance.

Slightly more forward in pseudorapidity are the end-cap calorimeters. The EM end-cap (EMEC) covers the range $1.375<|\eta|<3.2$, and it is very similar in construction and design to the EM barrel calorimeter. The only major difference between the two is the cryostat in which they are placed. The total depth of the EMEC is $26 \mathrm{X}_{0}$. Located directly behind the EMEC is the the hadronic end-cap calorimeter (HEC), covering the range $1.5<|\eta|<3.2$. It consists of two coaxial wheels, placed beside one another, on either side of the detector. The HEC uses LAr as the active medium and copper plates as absorbers. It has granularity $0.1 \times 0.1$ in the region $1.5<|\eta|<2.5$, and $0.2 \times 0.2$ in $2.5<|\eta|<3.2$.

In the most forward region of the detector lies the forward calorimeter (FCal), covering the range $3.1<|\eta|<4.9$. It consists of three, $45 \mathrm{~cm}$ long, cylindrical modules, placed beside one another, surrounding the beam line. Each uses LAr as the active 
medium. The first module (FCal1) is optimised for electromagnetic measurements using copper as the absorbing material. The other two modules, FCal2 and FCal3, act as hadronic calorimeters, and use the much denser tungsten for absorption. Behind the FCal3 module at each end is a plug of passive brass (Plug3) to help shield the muon system. Figure 3.4 shows a schematic of the three FCal modules and plug3.

The electrode design of FCal is unique in comparison to the other calorimeters in ATLAS. The modules have approximately 12000 holes drilled through them, running parallel to the beam line. Each hole is filled with a copper tube which houses a metal rod, which serves as the anode. The LAr is placed between the rod and tube, and a potential difference is applied allowing for charge collection at the anode. The geometry of the cells is non-projective, but because it is so far in the forward pseudorapidity region, the calorimeter tubes that run parallel to the beam line are approximately projective. The approximate granularity in $\eta-\phi$ is $0.2 \times 0.2$. The total thickness of FCal is approximately $10 \lambda$.

FCal is unsurpassed in its extensive detector coverage. No previous hadron collider detector has reached so far in forward rapidity. This is rather exciting from a physics perspective as it allows the ATLAS experiment to probe never-before reached areas of phase space. Part of the FCal was assembled at Carleton University and it is therefore Carleton's responsibility to monitor its performance and analyze the data it produces.

\subsubsection{Muon Detectors}

The final layer of the ATLAS detector is the Muon Spectrometer. This is a general feature of high energy collider detectors as muons, due to their large mass and absence 
Figure 3.4: Schematic of the ATLAS Forward Calorimeter. Image taken from Reference [3].

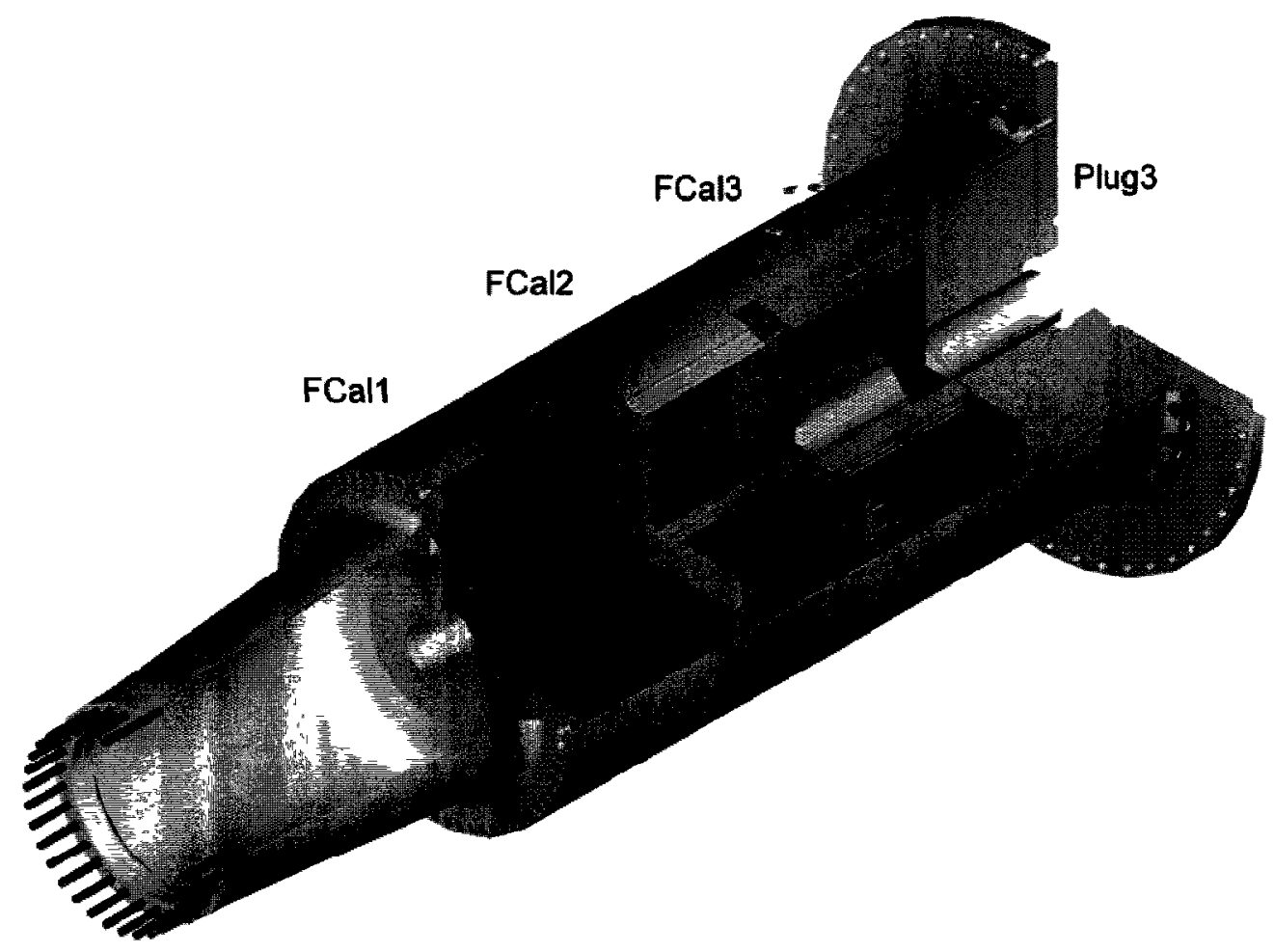

from strong interactions, penetrate deeper than any other particle ${ }^{1}$ The ATLAS muon system is simply a well shielded tracking detector which measures the momenta of the penetrating muons. This is achieved by applying a magnetic field to bend the muon trajectory and measure the curvature, hence momentum. The magnet system consists of three parts: the Barrel Toroid and two End-Cap Toroids. The barrel toroid surrounds the calorimeters and the end-cap toroids, providing magnetic bending over the range $|\eta|<1.4$. Its inner and outer radii are $4.7 \mathrm{~m}$ and $10.05 \mathrm{~m}$, respectively, giving enough room for precise momentum measurements. It consists

\footnotetext{
${ }^{1}$ This excludes neutrinos which are only weakly interacting hence practically undetectable in a typical collider detector, and taus which are very heavy and decay too quickly.
} 
of eight superconducting coils, with 120 turns per coil, all encased in large vacuum vessels. The nominal coil current is $20.5 \mathrm{kA}$ producing a magnetic field of $0.5 \mathrm{~T}$. The end-cap toroids are designed in a similar fashion as the barrel toroid, the major difference being their smaller size, with inner and outer radii of $0.825 \mathrm{~m}$ and $5.35 \mathrm{~m}$, respectively. The smaller size allows the end-caps to produce a magnetic field $1.0 \mathrm{~T}$. They span the region $1.6<|\eta|<2.7$; the region $1.4<|\eta|<1.6$ has magnetic bending provided by both the barrel and the end-cap toroids, albeit at a weaker strength.

\subsubsection{Trigger System and Data Acquisition}

The triggering system, activated by a sufficient energy deposit in the detector, is used to select interesting collision events. It consists of three distinct levels: L1, L2, and the event filter $(\mathrm{EF})$. The L1 trigger searches for high $p_{T}$ objects, such as electrons, photons, muons, jets, missing $E_{T}$, etc. It uses minimal information from regions of the detector and makes a decision in less than $2.5 \mu \mathrm{s}$, accepting events at a rate of about $75 \mathrm{kHz}$. These events are then analysed by the L 2 trigger, which uses slightly more information from the detector making a decision in about $40 \mathrm{~ms}$. This reduces the acceptance rate to about $3.5 \mathrm{kHz}$. Finally, the EF trigger analyses events passing L2 in more detail, using offline analysis procedures and makes a decision whether or not to record the event in about $4 \mathrm{~s}$. This brings the final event acceptance rate to about $200 \mathrm{~Hz}$.

Due to the high luminosity of the LHC, the interaction rate is higher than the maximum event-acceptance rate. The bandwith capacity is too low to transmit and record every single event. For this reason the majority of the interaction events are discarded. In any case, most of these events will correspond to soft scattering of the 
protons with no potential for interesting physics studies, such as those discussed in Section 3.3. Low $p_{T}$ events may activate certain triggers, but they are not generally recorded. Each trigger has a prescale, a number that conveys how many events will be recorded by the trigger. If the prescale is $\mathrm{X}$, then one out of $\mathrm{X}$ events will be recorded. Hence triggers with large prescales discard most of the events collected. Large prescales $(\mathrm{X})$ are imposed on triggers activated by low $p_{T}$ events.

For each trigger level, numerous specific triggers are available which are designed to detect certain particles or activity in the detector. Of these, the most worthy of mention, for the analyses presented in this thesis, are the Minimum Bias Trigger Scintillator (MBTS) and Calorimeter (Calo) trigger.

\section{Minimum Bias Trigger Scintillator (MBTS)}

The MBTS trigger system, as the name implies, is used to select virtually any kind of event arising from inelastic p-p interactions. It uses 32 scintillator counters covering the range $2.09<|\eta|<3.84$. The passage of charged particles produces scintillating photons, which activates the trigger. The trigger is primarily used for analysing jets, or other objects, with relatively low $p_{T}$, typically below $40 \mathrm{GeV}$. Since these occur very frequently the MBTS has the highest prescales of all triggers.

\section{Jet (J) Triggers}

The $\mathrm{J}$ trigger fires on any significant energy deposit in the calorimeters, and is therefore primarily used to signal the presence of jets. There are various different $p_{T}$ thresholds for this trigger. For example, the $\mathrm{J} 5$ trigger requires at least $5 \mathrm{GeV}$ of energy to be deposited in a particular region of interest (ROI) of the calorimeter, the 
J30 requires $30 \mathrm{GeV}$ and so on. A jet is generally composed of many ROI's, so the jet $p_{T}$ threshold is many times higher than the energy deposit per ROI. For example, the $\mathrm{J} 5$ trigger is fully efficient for jets with $p_{T}$ above roughly $40 \mathrm{GeV}$.

The central jet trigger is separate from the forward jet (FJ) trigger. The former picks out jets in the range $|\eta|<3.2$, while the latter spans $3.2<|\eta|<4.9$. 


\section{Chapter 4}

\section{Calorimetry and Particle}

\section{Showering}

The key physics objects being analysed in this thesis are jets. In high energy physics experiments jets are observed and measured using calorimeters. This chapter describes the relevant background information to understand calorimetry and the physics processes within them.

\subsection{Calorimeter Showers}

As a particle traverses the dense material of a calorimeter, the interaction between the particle and the material will produce multiple new particles of lower energy; each of these then interacts with the material in a similar way, a process that continues until numerous low energy particles are produced. This cascade of secondary particles is called a shower. The shower continues to grow until all of the energy is contained in the calorimeter, i.e. the final particles produced do not have sufficient energy to 
create new particles.

Showers can be classified according to the nature of the particles present. There are two important types of showers: electromagnetic and hadronic.

\subsubsection{Electromagnetic Showers}

Electromagnetic (EM) showers are initiated by either photons or electrons/positrons. The only interaction present between the material atoms and the newly generated shower particles is electromagnetic, hence the name. There are several different processes that may take place to produce new particles. For photons, three different processes can occur: pair production, Compton scattering, and photoelectric effect. Pair production is the conversion of a photon into an electron/positron pair and it is the most likely process at high energies [11], i.e. energies above twice the electron rest mass $\left(2 m_{e}\right)^{1}$. At energies below $2 m_{e}$, the most dominant process becomes Compton scattering; a process where a photon scatters off an electron and transfers a significant amount of its energy to it. Finally, at lower energies, below a few hundred keV, the photoelectric effect starts to dominate. This is a process where a photon transfers all of its energy to knock an electron out of atomic orbit.

Each of the three photon processes, discussed above, produces new electrons, and in the case of pair production, an electron/positron pair. As electrons and positrons traverse matter, they lose energy and produce new particles via three processes: bremsstrahlung, ionization, and Cerenkov radiation. Bremsstrahlung, German for 'breaking radiation' is the radiation of photons by charged particles as they deceler-

\footnotetext{
${ }^{1}$ In this discussion the natural unit system is adopted. It is a system of units where the speed of light, $c$, and Planck's reduced constant, $\hbar$, are both set to one and are unitless. Hence an energy of $2 m_{e}$ is really $2 m_{e} c^{2}$ in the SI system.
} 
ate. This is the dominant process at high energies. At energies below a few $\mathrm{MeV}$, ionization becomes dominant, where the traversing electron/positron transfers a part of its energy to the material electrons, kicking them out of atomic orbit. If the energy imparted onto the atomic electrons is insufficient to kick them out of orbit, the atoms may be temporarily excited and emit photons as they return back to their ground state. If the traversing electron/positron travels at a speed greater than the speed of light in the material, the material atoms will emit Cerenkov radiation. The energy loss due to Cerenkov radiation is smaller than that of bremsstrahlung and ionization.

One can imagine a typical EM shower initiated by, say, a high energy electron. Upon encountering the calorimeter, the electron produces several photons through bremsstrahlung. Some of the photons may be energetic enough to pair produce, while others Compton scatter, introducing new electrons/positrons into the shower. These will then undergo their interactions, producing more photons and so on. As the shower develops, the average energy of the shower particles decreases, and at some point no further multiplication takes place. The depth at which this takes place is called the shower maximum. Beyond that point, the shower photons cease to pair produce, and the electrons are more likely to lose their energy through ionization than to radiate new photons. The number of shower particles as well as the amount of energy deposited in a given thickness of the calorimeter gradually decreases and eventually the shower comes to a stop.

With electromagnetic showers, all of the initial energy of the incoming photon or electron/positron is accounted for, provided that the calorimeter is thick enough to contain all of the shower particles. In other words, all of the energy is ultimately used to generate electric charge, which is then read out as signal and converted into 
an energy measurement.

\subsubsection{Hadronic Showers}

Hadronic showers are much like the EM counterparts but with some key substantial differences. The nature of a hadronic shower is much more complicated than that of an electromagnetic one. The key difference between the two is that with hadronic showers more interactions are at play in addition to the EM interaction. Most notably, the strong interaction plays an important role in the shower development. Moreover, the weak interaction also has a non-negligible contribution to the shower characteristics.

A hadronic particle is required to initiate a hadronic shower. There are many different species of hadrons, some electrically charged and some neutral. When an energetic hadron encounters the atoms of a calorimeter, a vast variety of processes can occur. If the hadron is charged, it will traverse the material leaving it ionized, in a continuous stream of events. At some depth, however, the hadron will interact strongly with the atomic nuclei. The most likely process to occur here is nuclear spallation - the dissociation of the nucleus by the impinging hadron. With nuclear spallation a myriad of different events can occur. The hadron itself may change dramatically; it may, for example, turn into several new hadrons. The nucleus also changes its identity, losing neutrons and protons, and ending up in a highly excited state radiating photons.

Most of the newly produced hadrons are unstable mesons and hence quickly decay into other particles. Being the lightest, the most copiously produced meson is the pion, which itself comes in three varieties: one neutral and two charged with charges $\pm 1 e$, where $e$ is the magnitude of the electron charge. The neutral pion, $\pi^{0}$, decays 
electromagnetically, practically instantly, into two photons $\left(\pi^{0} \rightarrow \gamma \gamma\right)$. On average, roughly one-third of the produced mesons are $\pi^{0} \mathrm{~S}[10]$. Therefore, hadronic showers generally contain a component that propagates electromagnetically.

Charged pions are more stable and hence have a longer lifetime. They generally penetrate a substantial amount of the calorimeter, depositing energy through ionization. At some point, however, they decay weakly into a muon and a neutrino. This is how the weak interaction manifests itself in hadronic showers. The neutrino only interacts weakly, therefore it does not deposit any energy in the calorimeter. The muon deposits energy through ionization, but it also escapes the calorimeter, being the most penetrating of all particles.

Pions are only an example of the types of hadrons present in a shower. Other heavier mesons are also possible, with lower abundance but similar characteristics. Baryons are, of course, also present. Neutrons are relatively long lived and electrically neutral, so nuclear interactions are the only mechanism for their energy loss and contribution to shower development.

With electromagnetic showers, deposition of energy in a calorimeter and signal readout is trivial. In the hadronic case, however, this is much more complicated. Four different components of energy-flow exist within a hadronic shower: EM visible energy, non-EM visible energy, invisible energy, and escaped energy.

EM visible energy is simply the electromagnetic component of the shower arising from $\pi^{0} \rightarrow \gamma \gamma$. As in an EM shower, this energy is fully accounted for and registered in the calorimeter. It is called $E M$ energy because it originates from photons which are also present in EM showers. Roughly $35 \%$ of the energy in a hadronic shower is EM energy. 
Non-EM visible energy is the energy that is deposited and registered in the calorimeter, but arises from particles not present in EM showers, such as charged mesons, protons, muons etc. These are electrically charged particles, so they can continuously deposit energy through ionization. Approximately $35 \%$ of shower energy is non-EM.

Invisible energy is simply as the name implies - energy deposited by the shower that can not be measured nor detected by the calorimeter. It arises from the nuclear interactions between the penetrating hadrons and the atomic nuclei of the calorimeter. As mentioned, nuclear spallation is the most common process in these types of interactions. To dissociate a nucleus and to overcome the binding energy of the constituent nucleons requires a significant amount of energy. Since the binding energy is associated with the strong/nuclear force, this energy is not visible to a calorimeter, which is a device that operates via the electromagnetic interaction (an electronic device). Invisible energy comprises roughly $25 \%$ of the shower energy.

Lastly, escaped energy is the part of the shower that escapes the calorimeter. The particles responsible for this are the elusive neutrinos and the highly penetrating muons. On the order of a few percent of the shower energy escapes the calorimeter.

Figure 4.1 shows a simple schematic of a typical hadronic shower, including the different energy components. 
Figure 4.1: A simple depiction of a hadronic shower, highlighting the possible ways in which energy can be dissipated. Refer to text for more details. Image courtesy of Sven Menke, ATLAS Collaboration.

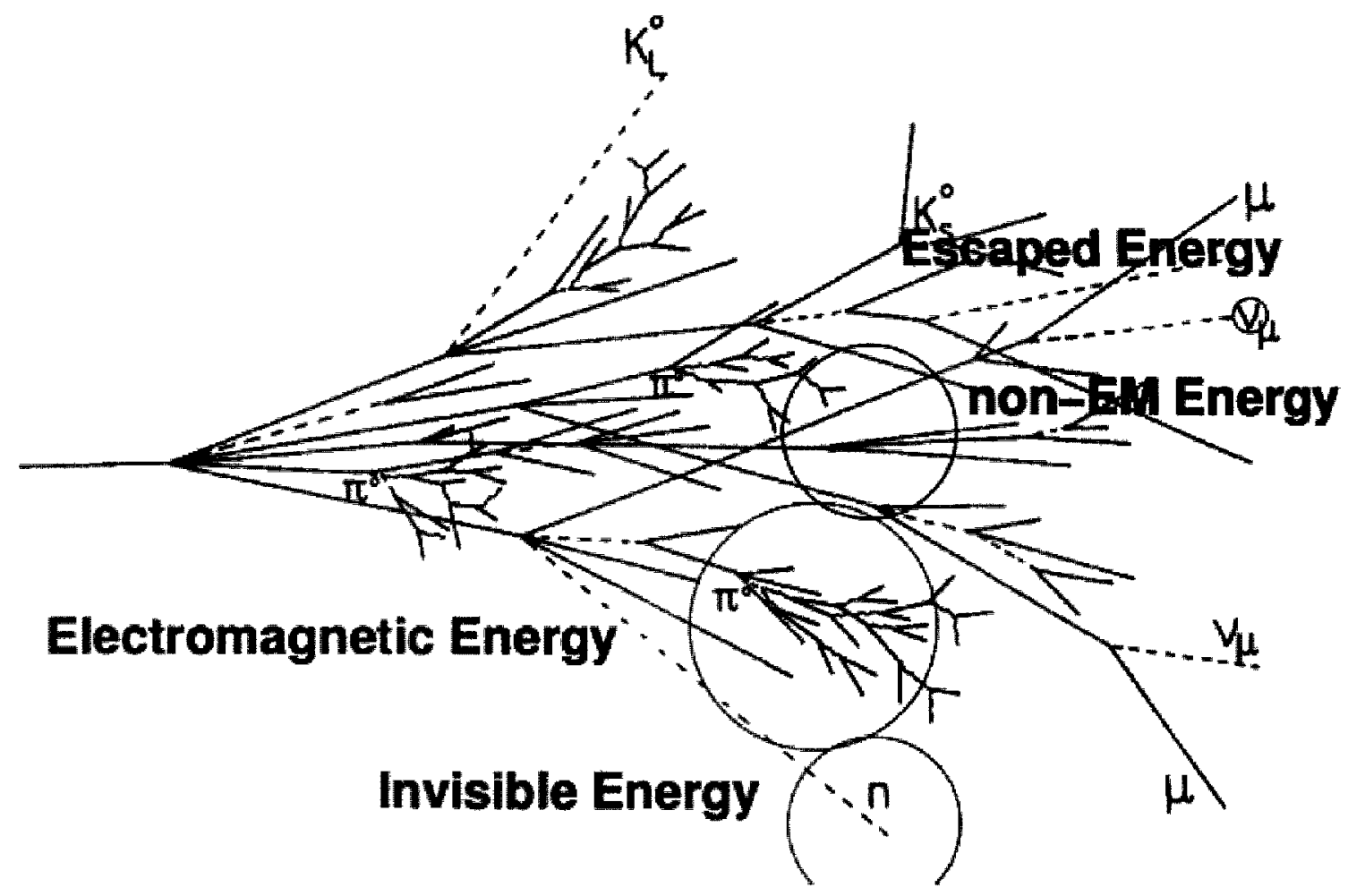




\section{Chapter 5}

\section{Jet Reconstruction in ATLAS}

\subsection{Introduction}

While the production of jets was briefly discussed at the end of Chapter 2, the next step is to describe how the actual jets are identified and reconstructed in the ATLAS detector. This, however, is an ever-changing process as both beam and detector conditions are constantly changing. Moreover, the performance of the reconstruction can always be improved with time. The process described here corresponds to the strategy put in place for jet reconstruction during the 2010 data-taking period.

Jet reconstruction is a multi-step process starting from raw energy clusters and electronic signals in the calorimeter, where a jet algorithm collects the input and builds a reconstructed (reco) jet object. The reco jet energy is corrected for excess energy deposits due to additional p-p interaction products, followed by an essential calibration that restores the expected truth jet 4-vector momentum, independent of detector used. The final reco jet has, on average, all of its 4-vector momentum variables, $\left(E, p_{x}, p_{y}, p_{z}\right)$, as close as possible to those of the truth jet. 


\subsection{Jet Reconstruction Input Objects}

Many of the individual particles that make up a jet carry electric charge. As a jet traverses a calorimeter, the charged particles will produce ionization charge (or scintillating photons) leaving an electronic signal in the calorimeter. Calorimeters are composed of cells and each cell reads out a signal. The signal can be easily converted into an energy deposit $(E)$ measurement. Cells with a significant energy deposit are then grouped together to form clusters, the main input of jet algorithms. In ATLAS, clusters are created from cells following a 3-step '4-2-0' noise-suppression technique:

1. A seed cell having the highest energy deposit and $|E|>4 \sigma_{\text {norse }}$ is located.

2. All surrounding cells with $|E|>2 \sigma_{\text {nosse }}$ are added.

3. One last layer with $|E|>0 \sigma_{\text {nonse }}$ is included to form the cluster.

Here $\sigma_{\text {nonse }}$ is the standard deviation of the electronic noise in the calorimeter. The absolute value of $E$ is used because a cell could yield a negative energy measurement; the readout of a cell fluctuates around a baseline value which corresponds to $E=0$. Figure 5.1 illustrates the method for cluster formation in a 2-D mapping.

\subsection{The Anti- $k_{T}$ Jet Algorithm}

The anti- $k_{T}$ jet algorithm [12] uses calorimeter clusters, described above, as input objects. All the clusters are iteratively paired together within a given geometrical acceptance to form reconstructed jets. For each input object $i$, the quantities $d_{\imath \jmath}$ and 
Figure 5.1: 2-Dimensional illustration of cluster formation, in an $(\eta, \phi)$ map, from individual cells in the ATLAS calorimeter. The coloured cells in the middle form a cluster while the dark grey at the bottom left do not. Image adapted from P.O. DeViveiros, ATLAS Collaboration.

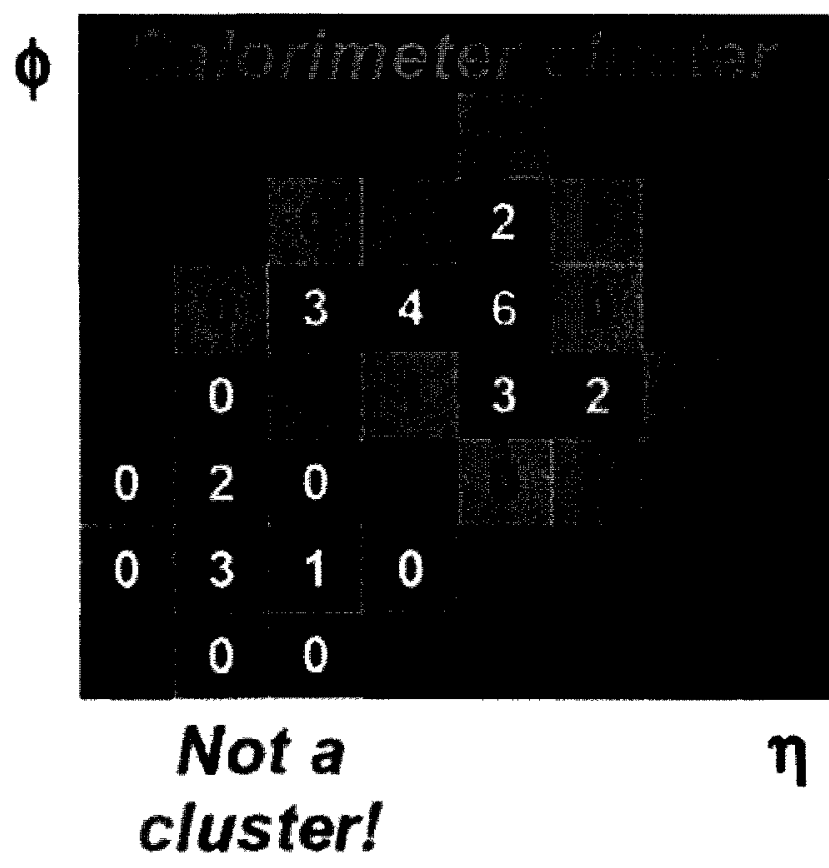

$d_{i B}$ are constructed:

$$
\begin{array}{r}
d_{i j}=\min \left(k_{T, i}^{-2}, k_{T, j}^{-2}\right) \frac{(\Delta R)_{i j}^{2}}{R^{2}} \\
d_{i B}=k_{T, i}^{-2}
\end{array}
$$

where

$$
(\Delta R)_{i j}^{2}=\left(y_{i}-y_{j}\right)^{2}+\left(\phi_{i}-\phi_{j}\right)^{2},
$$


while $k_{T, \imath}$ is the transverse momentum $\left(p_{T}\right)$ of object $i, \phi$ is the azimuthal angle, and $y$ is the rapidity ${ }^{1}$, defined as

$$
y=\frac{1}{2} \ln \left(\frac{E+p_{z}}{E-p_{z}}\right)
$$

where $\mathrm{E}$ denotes the energy, and $p_{z}$ is the component of momentum along the beam line. The clustering parameter $R$ can be seen intuitively as the radius of the cone jet in $y-\phi$ space. ATLAS currently uses two default jet sizes, $R=0.4$ and $R=0.6$, set by the expected size of jets from p-p collisions at the LHC.

The iterative pairing of clusters to form reco jets is carried out as follows. A list containing all the $d_{\imath \jmath}$ and $d_{\imath B}$ values is compiled. If the smallest entry is a $d_{\imath \jmath}$, objects $i$ and $j$ are combined, i.e. their 4 -vector momenta are combined, to form a single $d_{\imath B}$ object, and the list is remade. If the smallest entry is a $d_{\imath B}$, this object is promoted to a jet and is removed from the list. Evidently the algorithm favours small objects, corresponding to large transverse momenta set by the inverse square of $k_{T}$. Moreover, if two objects are close together, i.e. their separation $\Delta R$ is less than $R$, then these will tend to be combined and comprise the same jet.

Each cluster is assumed to be massless, and the mass of the jet is calculated as

$$
M_{\text {jet }}=\sqrt{\left(\sum E_{\imath}\right)^{2}-\left(\sum \overrightarrow{p_{\imath}}\right)^{2}}
$$

where the summations run over all clusters forming the jet. The detector level angular coordinates $\eta$ and $\phi$ are taken from the energy centroid of the reco jet. With the anti$k_{T}$ jet algorithm, the 4 -vector momentum of the jet is built, at the electromagnetic

\footnotetext{
${ }^{1}$ For massless particles, rapidity and pseudorapidity are equivalent.
} 
(EM) scale, i.e. from the pure electronic detector signal. As discussed in more detail in Section 5.4 below, this is insufficient to accurately reconstruct the true jet energy, and more generally, the true 4-vector momentum of the jet.

There are other varieties of jet algorithms available [12], anti- $k_{T}$ being the default choice in ATLAS. Furthermore, the anti- $k_{T}$ algorithm can take other input objects

besides clusters. For example, it is possible to input calorimeter towers, which are slices in $\eta-\phi$ space that extend through the depth of the calorimeter. The focus has been put on clusters because they are used exclusively for the jets analysed in this thesis.

\subsection{Jet Calibration}

Jets are mostly hadronic in nature and as such they deposit energy in a calorimeter through hadronic showering. This causes a particular challenge in measuring the energy of a jet. Since calorimeters are electronic devices they cannot account for all of the jet's energy. Calorimeters measure energy by collecting ionisation charge or scintillating photons produced through the EM interactions of the traversing charged particles with the electrons of the absorbing material. As discussed in detail in Section 4.1.2, hadronic showers, and hence jets, have a significant fraction of their energy that is neither measurable nor detectable by calorimeters. This means that a manual calibration is required to restore the correct energy, and more generally, the correct 4 -vector momentum of a jet. The calibration constants are therefore scaling factors that are derived using simulated jets from Monte Carlo (MC) samples, comparing truth jets to reconstructed (reco) jets.

There are two energy scales for jets: the EM scale and the jet energy scale (JES). 
The EM scale energy of a jet is simply the energy that the calorimeter reads out from the deposited charge. This does not represent the true energy as it neglects the energy component of a jet which does not leave a trace in the calorimeter, i.e. the invisible and escaped energy (see Section 4.1.2). The jet energy scale (JES), on the other hand, represents the true jet energy. It is obtained by scaling the EM jet energy by a multiplicative constant, correcting for the invisible and escaped energy.

\subsubsection{Energy Calibration Constants}

The energy (E) calibration constants are multiplicative factors that restore the correct energy of a reco jet. This factor is simply the inverse of the jet response in a given bin of pseudorapidity $\eta$ and E. Figure 5.2 shows the jet response as a function of $\eta$ in different bins of $\mathrm{E}$. The jet response $(r)$ is simply defined as the ratio of the energy of the reco jet and the truth jet:

$$
r \equiv \frac{E_{j e t}^{r e c o}}{E_{j e t}^{t r u t h}}
$$

Hence the energy calibration constant $c_{E}$ is

$$
c_{E} \equiv \frac{1}{r} .
$$

Multiplying the reco jet energy by $c_{E}$, restores the correct energy of the jet. Once a jet has been calibrated with $c_{E}$, the jet is said to be at the 'EM+JES' calibrated energy scale. Figure 5.3 shows the correction factors as a function of jet $p_{T}$ in different bins of rapidity.

ATLAS employs a jet reconstruction threshold of $7 \mathrm{GeV}$ at the hadronic (cali- 
Figure 5.2: Simulated jet energy response in the ATLAS calorimeters as function of jet $\eta$ in different bins of energy. The shown intervals in $|\eta|$ correspond to those used in the jet energy scale uncertainty discussed in Chapter 7.

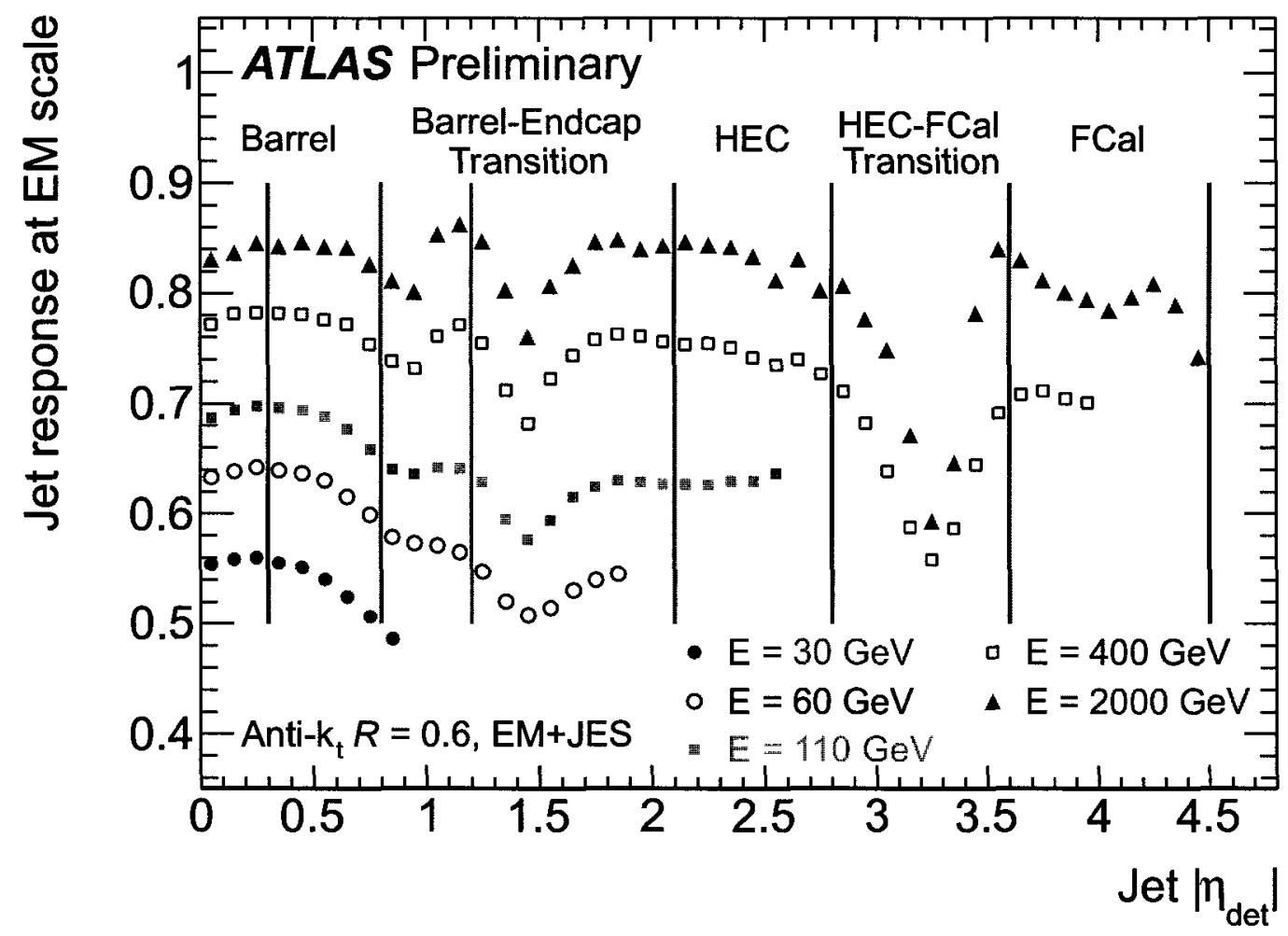

brated) scale. Jets below $7 \mathrm{GeV}$ are not saved in event data. This is necessary to free up space since the majority of jets produced have such low $p_{T}$. This, however, introduces extra complications when analysing low $p_{T}$ jet data.

\subsubsection{Pseudorapidity Calibration Constants}

As can be seen from Figure 5.2 the jet response varies significantly as a function of $\eta$. This is due to ATLAS itself; it is not a uniform detector, with many crack regions and varying amounts of material in front of the calorimeter. This not only biases 
Figure 5.3: Average Jet Energy Scale correction as a function of calibrated jet transverse momentum. The correction is only shown over the accessible kinematic range.

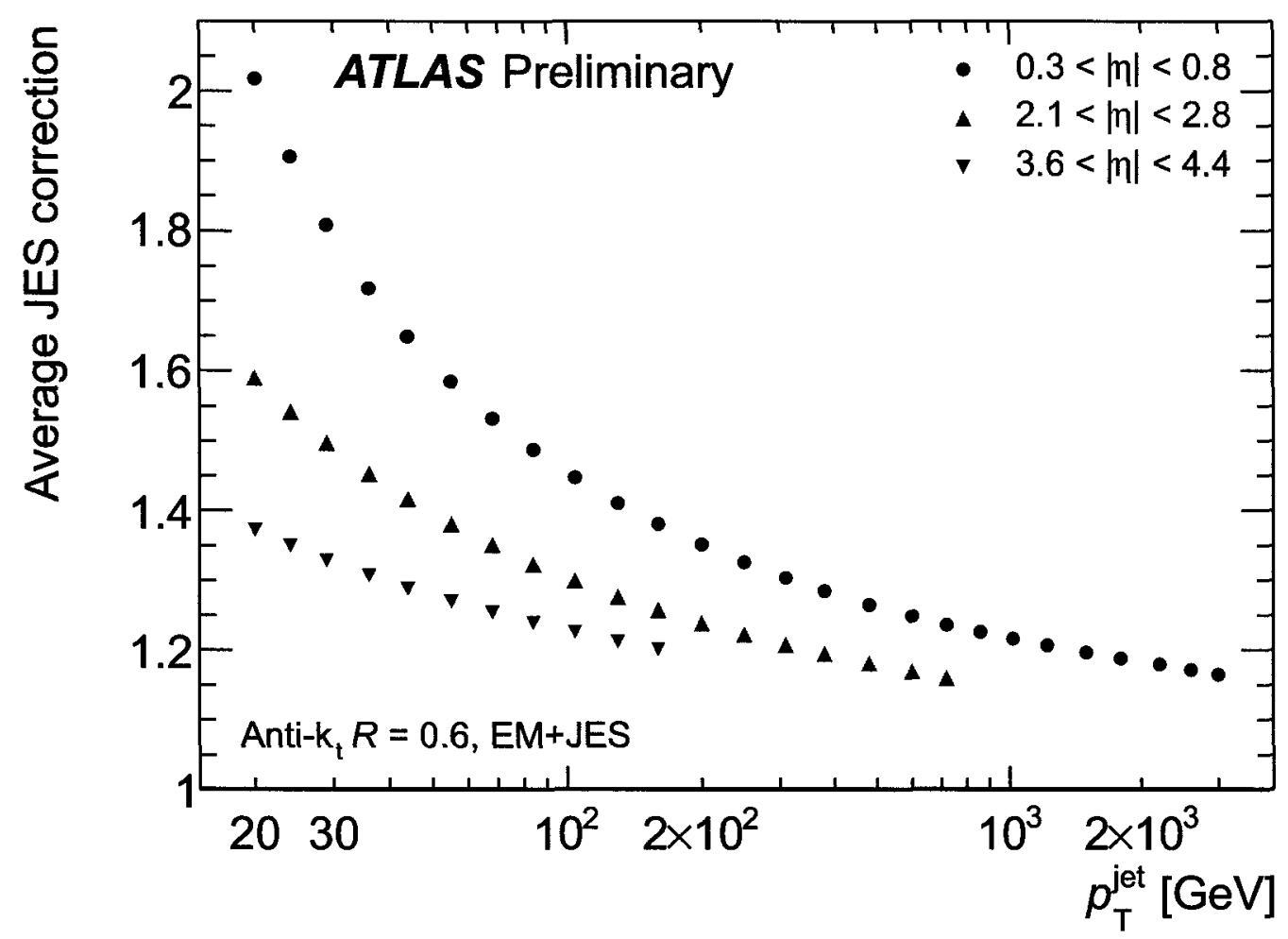

the jet energy measurement, but also the position $(\eta)$ measurement, which in turn translates into biased $E_{T}$ or $p_{T}$ measurements, since these are derived from $\eta$ :

$$
E_{T}=\frac{E}{\cosh (\eta)}
$$

and

$$
p_{T}=\frac{|\vec{p}|}{\cosh (\eta)}
$$

These are crucial variables for any physics analysis, hence a proper reconstruction of $\eta$ is essential. The $\eta$ calibration constant, $c_{\eta}$ is additive, obtained by comparing the 
$\eta$ of truth and reco jets:

$$
c_{\eta} \equiv \eta_{\text {reco }}-\eta_{\text {truth }}
$$

Evidently, the correct $\eta$ is restored by subtracting $c_{\eta}$ from the initially reconstructed $\eta$. Figure 5.4 shows the $\eta$ correction as a function of jet detector $\eta$, in different bins of energy. ATLAS is uniform in $\phi$, hence no correction is required for $\phi$ reconstruction.

Having proper measurements of energy, $\eta$, and $\phi$ allows for correct calculations of other variables such as mass and $p_{T}$, yielding an accurate overall reconstruction of the jet 4-vector momentum.

Figure 5.4: Average jet pseudorapidity correction constants as function of uncorrected (detector) jet $\eta$.

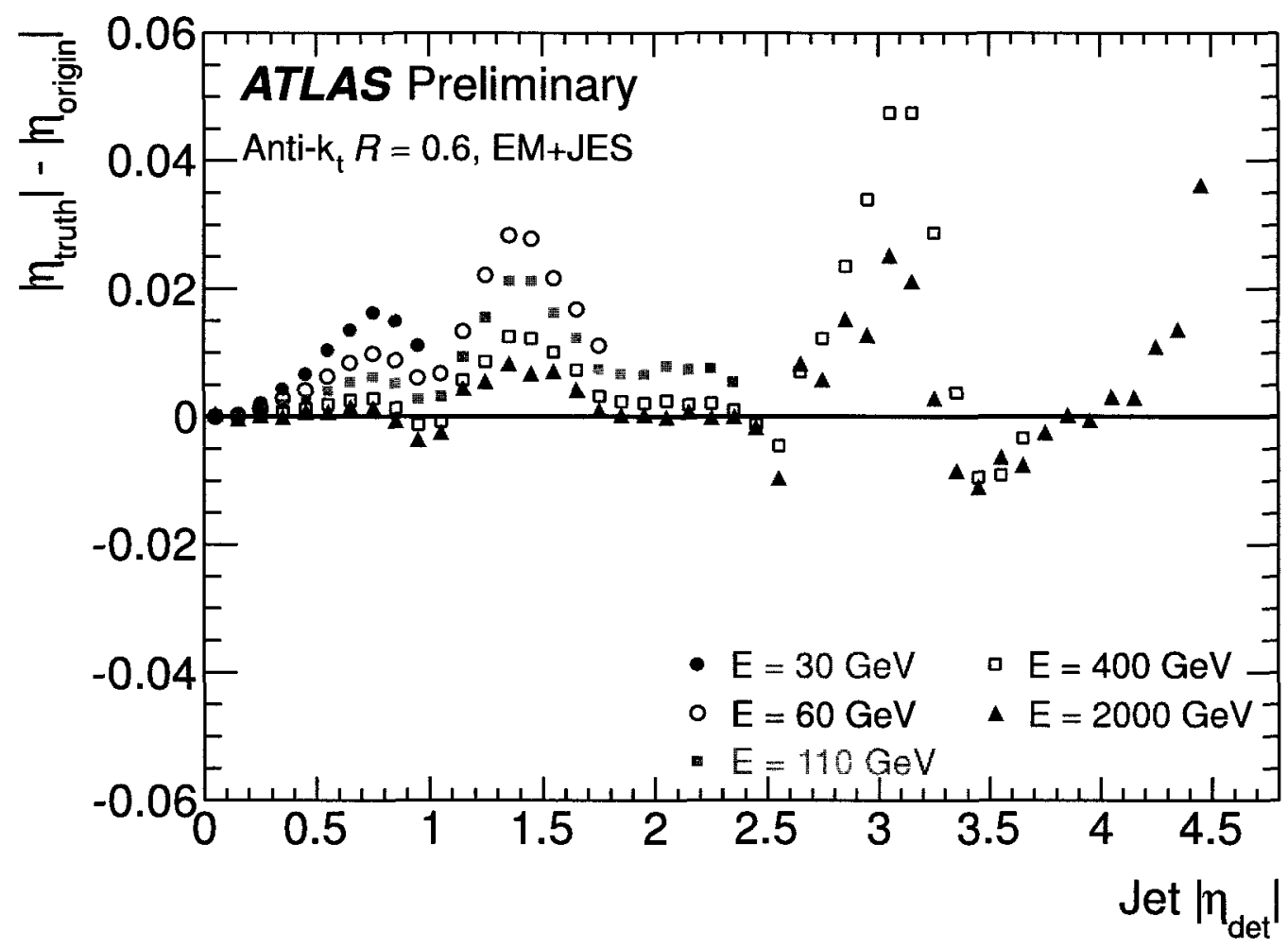




\subsection{Data-Driven Corrections}

\subsubsection{Pile Up}

In the high-luminosity environment of the LHC, several p-p interactions can take place in a given reconstructed event. Each such interaction has a primary vertex (PV) associated with it. Each of these PVs will have jets and other particles emanating from it. Each PV (or interaction) is independent from the others, hence so are the emanating particles and jets. However, many of the jets, coming from different PVs, may fall in the same region of the detector, overlapping with one another. This excess of activity in the detector is known as pile up and it clearly leads to inaccurate energy measurements. In ATLAS, the excess energy is corrected for in a simple manner [13]. For each number of PVs, calorimeter activity is analysed and an average energy density as a function of $\eta$ is observed. The excess energy in events with two or more PVs is subtracted from each jet. This is all done at the EM energy scale and therefore it is applied before the jet calibration.

\subsubsection{Origin Correction}

The location of the origin of the p-p interactions, the vertex, is designed to occur exactly at the center of the ATLAS detector. However, naturally there is some finite, non-negligible resolution of the vertex position along the beam axis. This position is referred to as the $\mathrm{z}$ of the primary vertex, since the beam line is along the $\mathrm{z}$-axis. The $\mathrm{z}$ affects the reconstruction of the jet $\eta$. In fact two eta's are used: detector $\eta_{\text {det }}$ and physics $\eta_{p h y s}$, where $\eta_{\text {det }}$ simply points back to the detector center and $\eta_{p h y s}$ is made to point back to the actual point of interaction, i.e. the origin is corrected. For 
physics analyses, $\eta_{p h y s}$ is used, but for certain detector performance analyses, such the $\eta$-intercalibration discussed in Chapter 6 , where the quantity of interest is the performance of the detector in specific regions, $\eta_{d e t}$ is the more appropriate choice.

\subsection{Summary}

To summarize, the reconstruction of jets in ATLAS follows a sequence of events:

1. Starting from the pure electronic signal in the calorimeter, the anti- $k_{T}$ algorithm is used to build jets at the EM scale.

2. Before the hadronic calibration constants are applied, excess energy due to pile up is subtracted, also at the EM scale.

3. The jets are made to point back to the primary vertex of the event, not the detector center. The detector center coordinates are also kept for certain analyses.

4. Lastly, the jet calibration constants are applied, restoring the final jet 4-vector momentum. 


\section{Chapter 6}

\section{Intercalibration Using Dijet}

\section{Balance}

\subsection{Introduction}

The ATLAS jet calibration constants, discussed in Chapter 5, are derived using Monte Carlo (MC) simulations. These constants need to be validated in-situ given the high complexity of the detector geometry and its material distribution, particularly in the forward region. In doing so, the calibration constants can be corrected if the need is present, and the uncertainty on the Jet Energy Scale (JES) can also be investigated. Intercalibration using dijet balance is a particular method used for achieving this, by calibrating separate regions of the detector, segmented in $\eta$, with respect to a specific region that is chosen as the reference. The central region is chosen as the reference, and the more forward regions are inter-calibrated with respect to it. It is an in-situ method that uses real collision data from the ATLAS detector, and compares the results to Monte Carlo simulation. 
The primary emphasis of intercalibration is to assess the calorimeter performance in the forward region $(|\eta|>2.5)$, where charged particle tracking is not available ${ }^{1}$. The intercalibration results provide a component of the JES uncertainty, which will turn out to be the largest in the forward region. Chapter 7 gives more detail about the full systematic uncertainty on JES.

The results shown here are from p-p collisions at a center-of-mass energy of $7 \mathrm{TeV}$, with an integrated luminosity of $37 \mathrm{pb}^{-1}$, collected during the full LHC run of 2010 .

\subsection{Dijet Balance Method}

The method of calibrating one region of the detector (probe region) with respect to a reference region relies on a fundamental law of nature - the conservation of momentum. Initially as the protons are travelling down the beam line, there is no net transverse momentum, i.e. $p_{T}^{i}=0$, where $i$ denotes initial. Consequently, after the collision, the same must also be true. The dijet balance method utilises events where two back-to-back jets are produced in the final state, having equal and opposite $p_{T}$. The relative calorimeter response of jets is quantified by the $p_{T}$ balance between the reference jet and the probe jet. The $p_{T}$ balance is characterized by the asymmetry $A$ [14], defined as

$$
A \equiv \frac{p_{T}^{\text {probe }}-p_{T}^{r e f}}{p_{T}^{\text {avg }}},
$$

where $p_{T}^{a v g}=\left(p_{T}^{\text {probe }}+p_{T}^{r e f}\right) / 2$. The reference region is typically chosen as the best region of the calorimeter in terms of performance; one with the highest granularity (resolution) and homogeneity. This is the central region of the barrel calorimeter,

\footnotetext{
${ }^{1}$ Tracking can also be used to evaluate calorimeter performance, but this work is not presented here.
} 
here chosen as $0.1<\left|\eta_{\text {ref }}\right|<0.6$. The very central region $(|\eta|<0.1)$ is excluded from the reference due to inhomogeneity of the detector at the point where the two sides of the barrel calorimeter meet. The reference region is assumed to be properly calibrated and all other regions are assessed against it.

The asymmetry can be used to measure the probe jet's relative response to the reference jet via the relation

$$
R_{\text {probe }}=\frac{p_{T}^{\text {probe }}}{p_{T}^{\text {ref }}}=\frac{2+A}{2-A}=\frac{1}{c_{\text {probe }}}
$$

in analogy with Equations (5.6) and (5.7). If both jets are properly calibrated this quantity should be consistent with unity. If not, the correction factor $c_{\text {probe }}$ can be used to correct the probe jet energy scale to the calibrated scale of the reference jet. The deviation from unity can also be taken as the uncertainty on the jet energy scale in the probe region, assuming perfect dijet events are analyzed. More detail on this will be given in Section 6.3.2.

\subsection{Event Selection}

\subsubsection{Data Pre-Selection}

All data that are analysed must pass certain selection criteria (cuts). The first selection criterion requires the events to belong to specific runs and run periods (luminosity blocks) in which the detector, trigger, and reconstructed physics objects have passed data-quality assessment and are deemed suitable for physics analysis. The runs satisfying this are part of what is known as the Good-Run-List (GRL). 
Next, events are required to pass certain triggers. Two trigger strategies are used for event selection: MBTS trigger, and the jet trigger (JX or FJX), where X denotes the ROI threshold (see Section 3.3.5). The MBTS triggers are used for analyzing the jet response for jets below $40 \mathrm{GeV}$. At least one hit in the scintillators is required for the event to be selected. The jet triggers are used for analyzing jets with $p_{T}$ above $40 \mathrm{GeV}$. Either the JX or FJX trigger is required to fire for event selection.

The events were subsequently selected as collision candidate events by requiring exactly one good primary vertex (PV), where a good PV is defined as having at least 5 tracks originating from it. Selecting events with only one PV ensures no pile up, and requiring at least 5 tracks ensures the vertex originates from a genuine p-p interaction.

\subsubsection{Jet Selection}

All events are required to have at least two jets. The leading two jets, ordered according to their transverse momentum magnitude, must pass some cleaning criteria [15], which guards against fake jets arising from out-of-time energy deposits such as noise bursts and/or cosmic ray showers. One of the two leading jets must fall in the reference region $0.1<|\eta|<0.6$.

\section{Selection of Events with Dijet Topologies}

In order for the dijet balance method to be valid, only two back-to-back jets can occur in the final state. These can be referred to as perfect dijet events, with a $2 \rightarrow 2$ topology. However, the vast majority of events will embody other phenomena, such as final state radiation, leading to more than 2 final state jets. Unfortunately, rejecting 
all such events would leave behind too few perfect events, with insufficient statistics to carry out the analysis. A compromise strategy is made to mimic perfect dijet events as closely as possible. In doing so, the following cuts are applied:

$$
p_{T}^{a v g}>20 \mathrm{GeV} ; \Delta \phi\left(j_{1}, j_{2}\right)>2.6 ; p_{T}\left(j_{3}\right)<0.15 p_{T}^{a v g}
$$

where $j_{i}$ denotes the $i^{\text {th }}$ highest $p_{T}$ jet in the event, and $\Delta \phi\left(j_{1}, j_{2}\right)$ is the azimuthal angle between the two leading jets. The $20 \mathrm{GeV}$ cut is required because of the 7 $\mathrm{GeV}$ jet reconstruction threshold in ATLAS; jets with transverse momenta below 7 $\mathrm{GeV}$ are not saved in an event. It is therefore impossible to firmly apply the third jet selection criterion when the $p_{T}^{a v g}$ is below roughly $47 \mathrm{GeV}$. For events with $p_{T}^{a v g}<47$ $\mathrm{GeV}$, it is not certain whether the third jet truly has $p_{T}<0.15 p_{T}^{\text {avg }}$. Evidently the event selection consists of events where perfect $p_{T}$ balance is not expected, even at the particle (truth) level. This implies that the relative jet response is not necessarily expected to be unity. For this reason, the uncertainty on the jet energy scale will simply be taken as the discrepancy between data and MC.

\subsection{Trigger Bias and Correction}

The existence of trigger prescales during data acquisition modifies the natural kinematic distributions of the data. This introduces a bias in dijet balance in the forward region. The central calorimeter (jet) triggers are separate and independent of the forward ones, the former spanning $|\eta|<3.2$, the latter covering $3.2<|\eta|<4.9$. Events with a probe jet in the forward region can be triggered and selected by either the central or the forward trigger, or both. If the prescales of the two trigger systems 
are equivalent, no bias occurs. However, if the prescales are not equivalent, then the trigger with the lower prescale will select more events leading to a possible bias. An event selected and triggered by the probe jet (reference jet) will tend to shift the asymmetry distribution in the positive (negative) direction. This is caused by the $p_{T}$ resolution of the reconstructed jets. To clarify this, consider dijet events triggered only by the forward trigger and in particular, consider events with a fixed truth level $p_{T}$ of the two leading jets. This particular forward trigger becomes fully efficient at some known value of jet $p_{T}$, referred to as the efficiency point. If the true jet $p_{T}$ of the dijet event is slightly lower than the efficiency point, then the trigger will tend to select events where the $p_{T}$ of the probe jet has fluctuated upward, but it will not select events with a downward-fluctuated probe jet $p_{T}$, leading to a positive shift in the asymmetry distribution. The same but opposite effect occurs for events triggered by the reference jet, leading to a negative shift in the asymmetry distribution.

When the asymmetry distribution is filled with events coming from both trigger systems, the events need to be weighted according to their prescale in order to obtain an unbiased distribution. For the analysis presented here, the weighting of events was carried out as follows. If the event is triggered by the probe jet only, assuming the central trigger prescale is larger than the forward, the assigned weight is simply the ratio of the prescales of the two triggers (central and forward):

$$
w_{f}=\frac{P_{c}}{P_{f}},
$$

where the subscripts $c$ and $f$ are for central and forward, respectively. If the event is triggered by the reference jet only, or if the event is triggered by both jets, then the assigned weight is one. More generally, events picked up by the trigger with the 
smaller prescale, or larger effective integrated luminosity, are scaled down to match the prescale of the trigger with the larger prescale, or smaller luminosity. The event weighting cancels out the two opposing effects and yields an unbiased asymmetry distribution.

\subsection{Monte Carlo Event Simulation}

The MC event generator samples were produced with PYTHIA 6.4.21 [16] at a center-of-mass energy of $7 \mathrm{TeV}$, using the ATLAS MC10 parameter tune [17] and the MRSTLO [18] parton distribution functions. The samples are QCD dijet events, which were generated in specific bins of parton transverse momentum produced by the $2 \rightarrow 2$ matrix element. This is necessary in order to obtain a reasonable number of events at high $p_{T}$ due to the steeply falling dijet cross section as a function of transverse momentum. The samples are then re-combined by applying event weights to each sample according to the cross section for that sample. The generator events are passed through the standard GEANT4 [19] simulation of the ATLAS detector and then fully reconstructed and analyzed with the same software used for the data processing.

\subsection{Results}

The analysis is performed in bins of probe jet $\eta$ and $p_{T}^{a v g}$. For each $\eta$ bin $i$ and each $p_{T}^{a v g}$ bin $j$, a distribution of the asymmetry is obtained. The relative jet response for that bin is then calculated from the mean of the distribution $\left\langle A_{i j}\right\rangle$, using Equation 
(6.2):

$$
R_{\imath \jmath}=\frac{2+\left\langle A_{\imath \jmath}\right\rangle}{2-\left\langle A_{\imath \jmath}\right\rangle} .
$$

The uncertainty on $\left\langle A_{\imath \jmath}\right\rangle$ is simply taken as the uncertainty on the mean of the distribution, namely $\sigma / \sqrt{N}$, where $\sigma$ is the standard deviation of the distribution and $N$ is the sample size. The uncertainty on $R_{\imath \jmath}$ is calculated using standard error propagation.

Figures 6.1 and 6.2 show the asymmetry distribution for a sample of the $p_{T}^{a v g}-\eta$ bins. Only jets with $R=0.6$ are shown; $R=0.4$ jets exhibit very similar behaviour. Figure 6.1 shows various pseudorapidity bins in the $50-60 \mathrm{GeV} p_{T}^{a v g}$ bin, whereas Figure 6.2 shows various $p_{T}^{\text {avg }}$ bins in the FCal, region $3.6<|\eta|<4.4$. By looking at the distributions in the forward calorimeter, one can notice that the mean asymmetry tends to be larger in data than in the MC.

The final step is to extract the relative jet response, Equation (6.2), from the asymmetry distributions. Figure 6.3 shows the relative jet response as a function of $|\eta|$ in different bins of $p_{T}^{a v g}$. The effects of the jet reconstruction threshold can be seen in the lowest $p_{T}^{a v g}$ bin, where the response drops more dramatically since the third jet may have up to $35 \%$ of $p_{T}^{a v g}$. In the higher $p_{T}$ bins, the response is more flat and closer to unity because the third jet is truly below $15 \%$ of $p_{T}^{a v g}$ after applying the appropriate cut. Figure 6.4 shows essentially the same information but displayed as a function of $p_{T}$.

The ratio of the relative jet response in data to that in MC is the quantity of interest; it is used as an estimate of the JES systematic uncertainty in a given $p_{T}-\eta$ bin. The results indicate that the JES, as studied via dijet balance, is understood to within roughly $10 \%$ in the most forward region (FCal), to within $8 \%$ in HEC-FCal 
Figure 6.1: Asymmetry distributions for the anti $-k_{T}, \mathrm{R}=0.6$ jets. Shown are several pseudorapidity bins in the $50-60 \mathrm{GeV} p_{T}^{\text {avg }}$ bin.
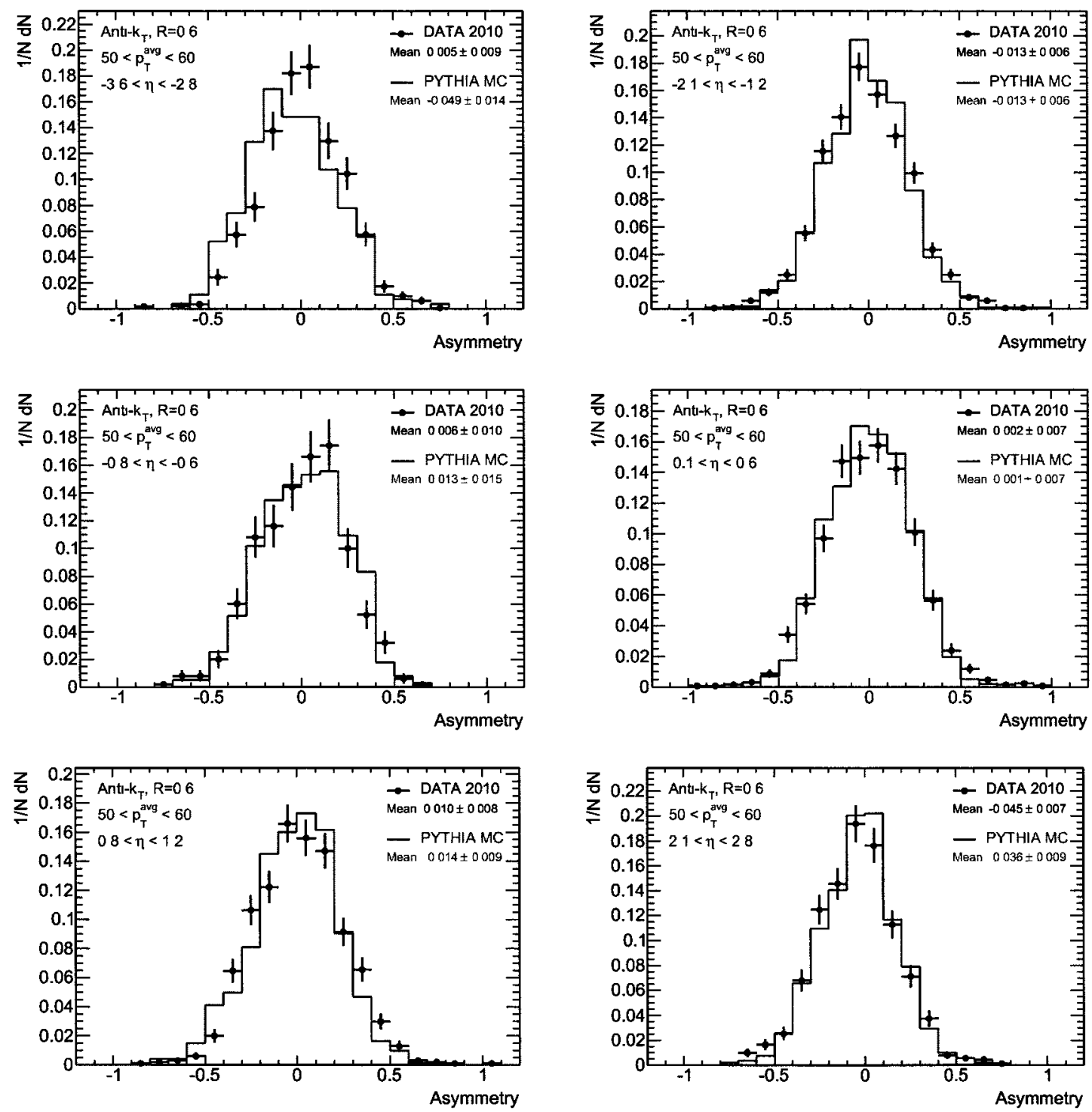

transition bin, and to within $5 \%$ in the more central regions, dropping to zero in region with $|\eta|<1$.

The uncertainty deduced from this method is only one of seven components of the JES uncertainty. The total uncertainty is obtained by a quadratic sum of all the 
Figure 6.2: Asymmetry distributions for the anti- $k_{T}, \mathrm{R}=0.6$ jets. Shown are several $p_{T}^{a v g}$ bins in the 3.6-4.4 pseudorapidity (FCal) bin.
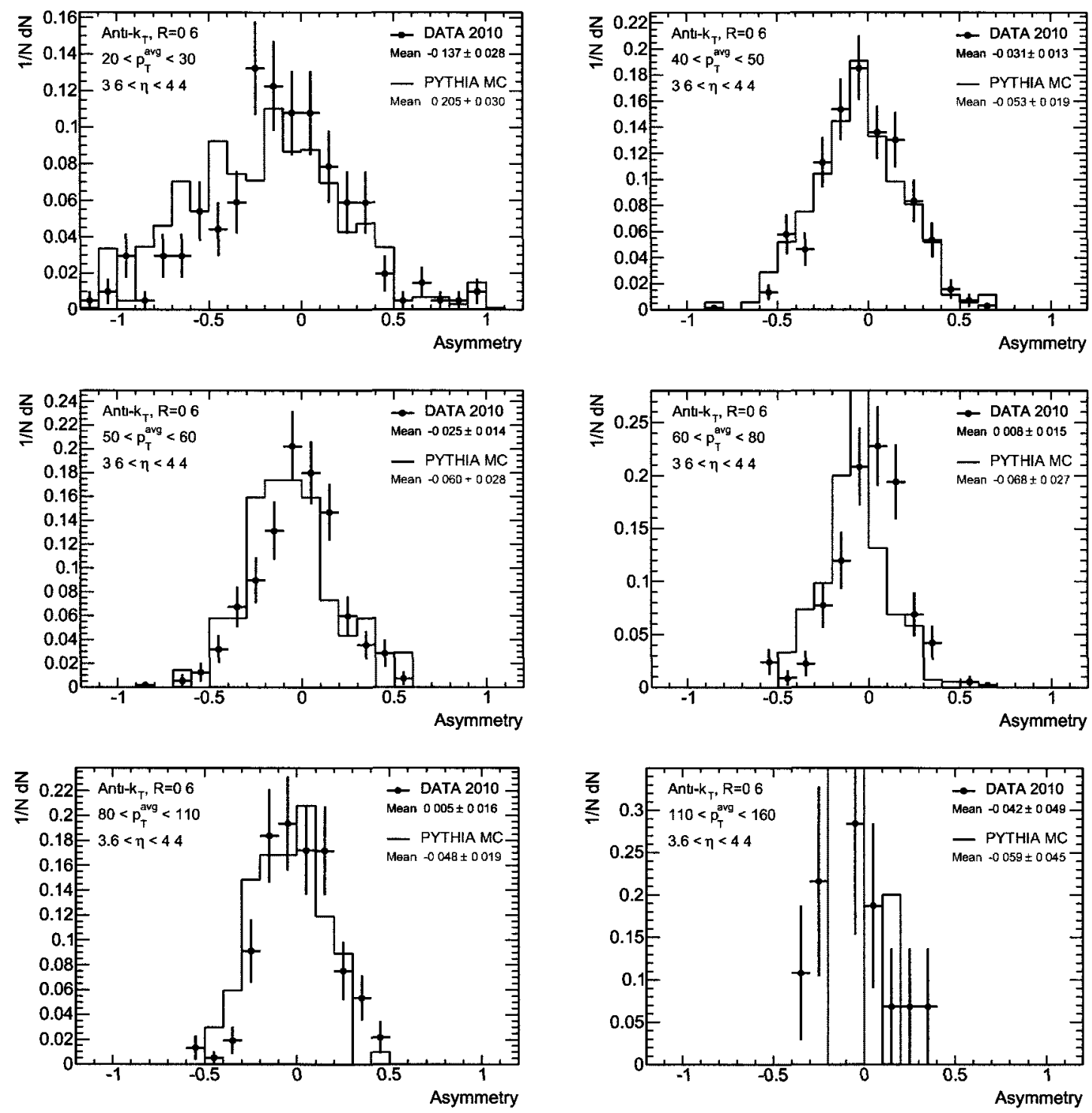

individual components. This is further discussed in Chapter 7. 


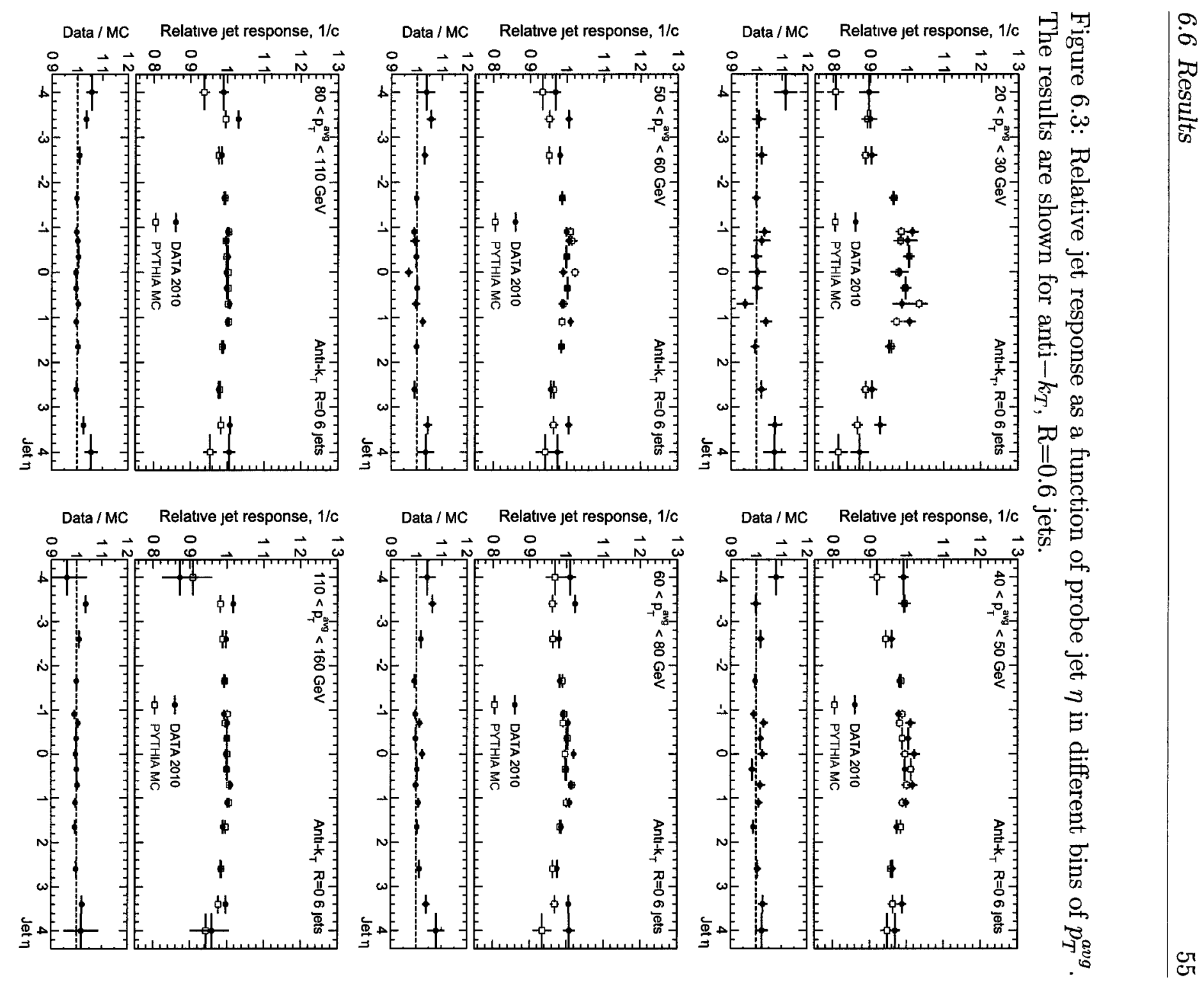


Figure 6.4: Relative jet response as a function of $p_{T}^{a v g}$ in different bins of probe jet $\eta$. The results are shown for anti $-k_{T}, \mathrm{R}=0.6$ jets.
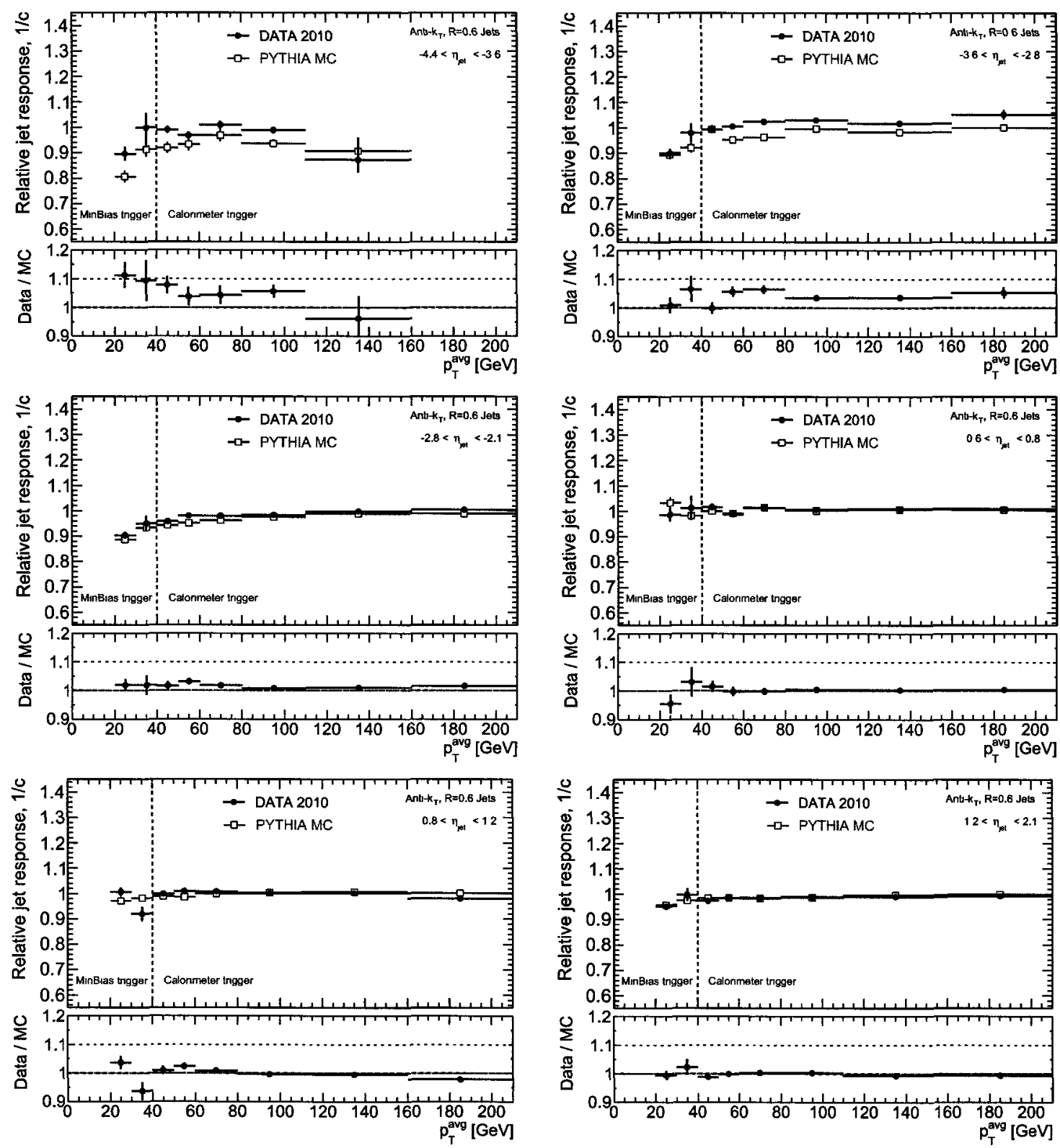

\subsection{Studies of the Underlying Event Modelling}

It is a peculiarity that such a significant disagreement between data and Pythia MC is observed in the relative jet response in the forward region. What could be the 
cause? A good first guess might be that the forward jets are mis-calibrated. If the MC does a poor job of simulating hadronic showering within the ATLAS detector, thereby mis-modelling the reconstructed (EM scale) energy and jet response, then the derived calibration will be wrong when applied to data. However, the disagreement observed here in the relative jet response is roughly $10 \%$ for low $p_{T}$ jets, as can be seen, for example, in Figure 6.3. This is quite a large disagreement. If calibration were off by $10 \%$, then it should be as easily seen when other kinematic distributions, such as $p_{T}$ spectra, are compared bewtween data and Pythia. The fact that no such large discrepancy is observed in other distributions implies that mis-calibration can not be the main cause of the disagreement observed here.

To gain insight into the discrepancy, it is beneficial to look away from the two leading jets in the event. Jets and energy deposits that arise from the underlying event, introduced in Section 2.4, tend to find themselves at very forward rapidities, close to the beam line. Such activity will often end up in the FCal and, therefore, will affect the relative jet response observed in the FCal. A study was carried out that looks at the energy deposits in the FCal away from the two leading jets. The sum of the transverse energy $E_{T}$ of clusters in slices of $\eta$ was considered in events that have both leading jets fall in the central region of the detector $(|\eta|<1.2)$. The data results were compared to two distinct Monte Carlo generators, Pythia [16] and Herwig [20]. Figure 6.5 shows the outcome of the study. It can be seen that Pythia disagrees with data in the modelling of the underlying event and all other general activity such as gluon radiation, that is away from the hard scatter jets. For completeness, data was compared to Herwig, and in this case also, the agreement is poor. It is interesting to note that Pythia predicts lower energy deposits away from 
the hard scatter, by roughly $20 \%$, while Herwig does exactly the opposite; Herwig overshoots data by roughly $20 \%$. This discrepancy is caused by the theoretical QCD modelling in the MC generators of the underlying event and other general activity away from the hard scatter jets. It is also the primary cause of the disagreement in the relative jet response between data and Pythia MC, and hence the cause of the large jet energy scale systematic uncertainty in the forward region.

Figure 6.5: The sum of the transverse energy of all clusters in a given slice of pseudorapidity as a function of $\eta$. The $\eta$ slices have width of 0.4 . The date results, collected by the minimum bias trigger, are compared to two distinct Monte Carlo generators, Pythia and Herwig.

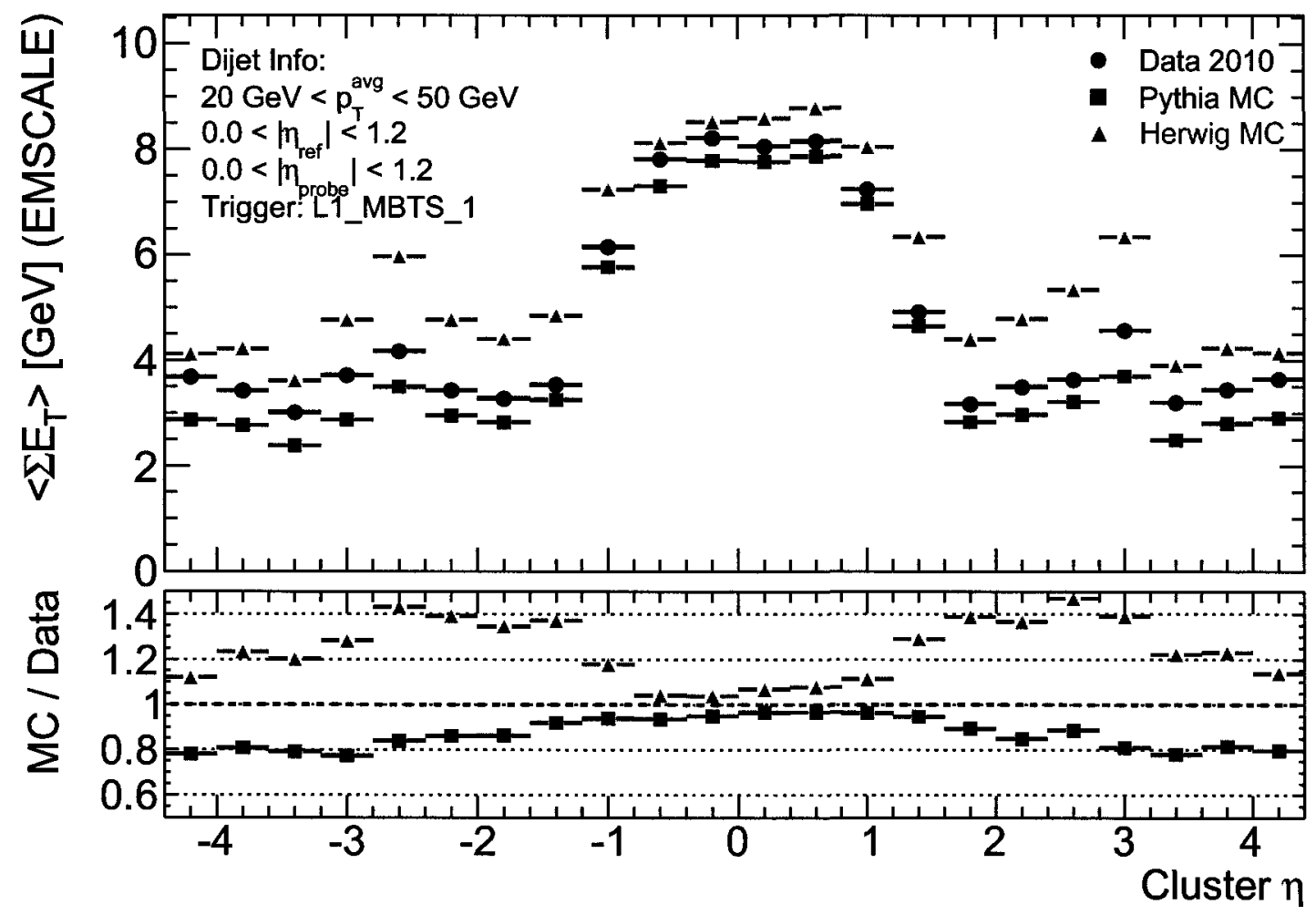




\section{Chapter 7}

\section{Jet Energy Scale Systematic Uncertainty}

\subsection{Introduction}

As jets are the most abundant products of the LHC's p-p collisions, a good understanding of their properties is necessary for many physics analyses. In particular, the uncertainty on the Jet Energy Scale (JES) is an essential ingredient for any such analysis. This chapter discusses the different components of the JES systematic uncertainty, describing the method used to obtain each one in order to calculate the total uncertainty. The intercalibration using dijet balance, presented in Chapter 6, is one such component from a total of seven. The other six are:

1. Non-closure from the MC JES calibration constants.

2. Uncertainties in the MC modelling.

3. Uncertainties from noise thresholds. 
4. Single particle tracking.

5. Uncertainties from material description.

6. Uncertainties due to Multiple Interactions.

\subsection{Non-Closure from JES Calibration Constants}

After the reconstructed jets in the nominal Monte Carlo sample are calibrated, the jet energy and $p_{T}$ response still show slight deviations from unity at low $p_{T}$. This effect is referred to as non-closure. Figure 7.1 shows the jet response for $p_{T}$ and energy as a function of $p_{\mathrm{T}}^{\text {jet }}$ for the nominal Monte Carlo sample in the barrel and endcap regions for anti- $k_{T}$ jets with $R=0.6$, where $p_{\mathrm{T}}^{\text {jet }}$ refers to the calibrated reconstructed jet.

Any deviation from unity (non-closure) in $p_{\mathrm{T}}^{\mathrm{jet}}$ and energy response after the application of the JES to the nominal Monte Carlo sample implies that the kinematics of the calibrated reconstructed jet are not restored to that of the corresponding particle jet. This is mostly due to the following:

1. There is an underlying assumption that every jet needs the same average correction (calibration) in a given jet $p_{T}$ bin. However, the derived calibration is taken as the mean of a distribution (filled with many jets) of the jet response. This means that the majority of the jets will get an incorrect calibration.

2. The same correction factor for energy and transverse momentum are used. In the case of a non-zero jet mass that does not reflect the truth jet mass, restoring only the jet energy and pseudorapidity will lead to a bias in the $p_{T}$ calibration. 


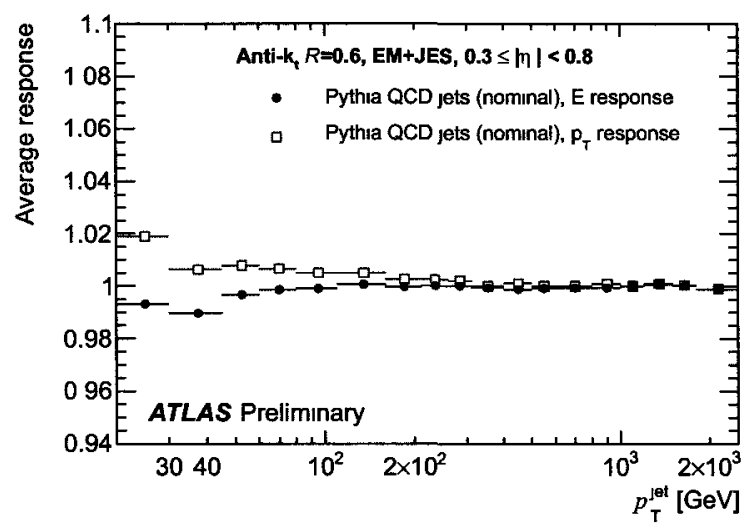

(a)

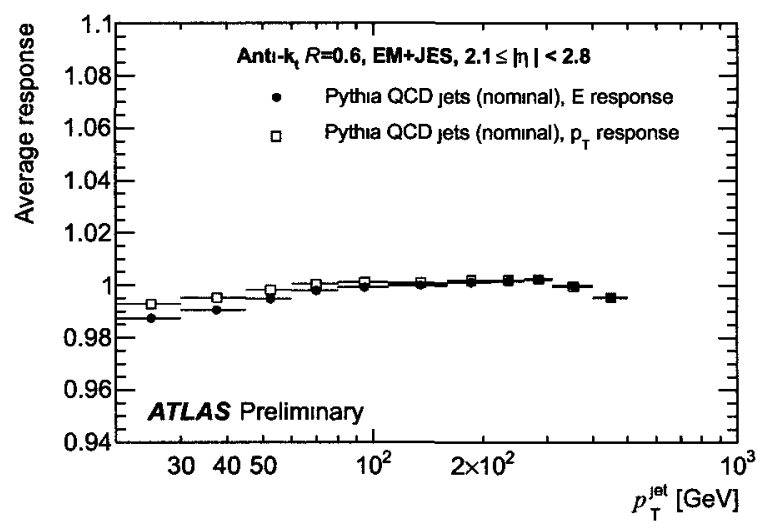

(b)

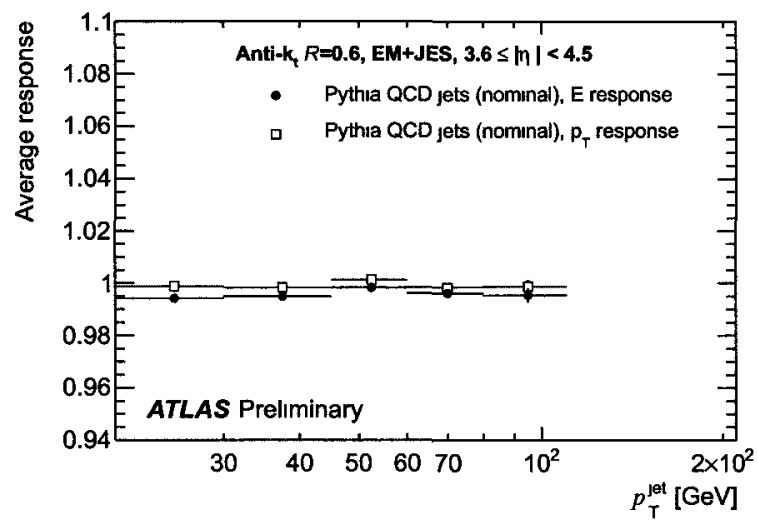

(c)

Figure 7.1: Simulated jet $p_{T}$ response (full circles) after the EM+JES calibration and jet energy response (open squares) as a function of $p_{\mathrm{T}}^{\text {jet }}$ for the nominal MC sample for jets in the central (a), endcap (b), and forward (c) calorimeter regions. 
The systematic uncertainty due to the non-closure of the nominal JES calibration is taken from the $\mathrm{MC}$ samples as the largest deviation of the response from unity between energy and $p_{T}$. In the barrel region $0.3<|\eta|<0.8$ this contribution amounts to about $2 \%$ at low $p_{\mathrm{T}}^{\text {jet }}$ and smaller than $1 \%$ for $p_{\mathrm{T}}^{\text {jet }}>30 \mathrm{GeV}$. In the endcap and forward region, the closure is better than $1 \%$ for $p_{\mathrm{T}}^{\text {jet }}>20 \mathrm{GeV}$, while the energy response is within $1 \%$ for jets with transverse momentum above $30 \mathrm{GeV}$. The deviation of the jet response from unity after calibration in the nominal MC sample is taken as the non-closure source of systematic uncertainty.

\subsection{Uncertainties in MC Modelling}

Several distinct Monte Carlo event generators are available, each operating with slightly different assumptions. Each tries to mimic physical reality as closely as possible, but discrepancies in the modelling are inevitable. The difference in the jet energy response between the different MC generators can be taken as a source of JES systematic uncertainty.

The baseline (nominal) Monte Carlo sample used to derive the jet energy scale and its systematic uncertainty is the PYTHIA event generator, briefly discussed in Section 6.5. It is compared to two others generators: ALPGEN [21] and PYTHIA PERUGIA [22]. These are referred to as test samples.

ALPGEN is a common event generator, one that uses more involved calculations of the matrix element; not only a $2 \rightarrow 2$ process as in PYTHIA, but also $2 \rightarrow 3$, $2 \rightarrow 4$, and $2 \rightarrow 5$ processes. For the LHC data taking in 2010, ALPGEN used the CTEQ6L1 parton distribution function [23], compared to the MRSTLO [18] set used in PYTHIA. Moreover, in ALPGEN, the fragmentation and the underlying event 
are implemented by HERWIG [24] and JIMMY [25], respectively, whereas PYTHIA does these on its own. PYTHIA PERUGIA is simply the PYTHIA generator tuned slightly differently. Namely the non-perturbative effects, such as hadronization and underlying event, are modified, including an increase in final state gluon radiation.

These distinct features of the MC modelling are bound to produce jets with a slightly different energy scale. Figure 7.2 shows the calibrated jet kinematic response for the two Monte Carlo generators and tunes used to estimate the effect of the Monte Carlo theoretical QCD model on the jet energy scale. The kinematic response of the nominal sample is also shown for comparison.

The ratio of the nominal response to the response of each of the two test samples is used as an estimate of the systematic uncertainty on the jet energy scale. The response in the test sample $\mathcal{R}_{\text {var }}$ and the response in the nominal sample $\mathcal{R}_{\text {nom }}$ are the starting points for the estimate of the JES uncertainty. The deviation of this ratio from unity is defined as:

$$
\Delta_{\mathrm{JES}}\left(p_{T}^{\mathrm{jet}}, \eta\right)=\left|1-\frac{\mathcal{R}_{\mathrm{var}}\left(p_{T}^{\mathrm{jet}}, \eta\right)}{\mathcal{R}_{\mathrm{nom}}\left(p_{T}^{\mathrm{jet}}, \eta\right)}\right|
$$

This deviation is calculated from both the energy and $p_{T}$ response, leading to $\Delta_{\mathrm{JES}}^{\mathrm{E}}\left(p_{T}^{\text {jet }}, \eta\right)$ for the deviation in the energy response, and to $\Delta_{\mathrm{JES}}^{p_{T}}\left(p_{T}^{\text {jet }}, \eta\right)$ for the deviation in the transverse momentum response. The largest $\Delta_{\mathrm{JES}}$ in each bin derived from the jet energy $(E)$ or transverse momentum $\left(p_{T}\right)$ response is considered as the contribution to the final JES systematic uncertainty due to the specific MC modelling systematic effect:

$$
\Delta_{\mathrm{JES}}\left(p_{T}^{\mathrm{jet}},|\eta|\right)=\max \left[\Delta_{\mathrm{JES}}^{\mathrm{E}}\left(p_{T}^{\mathrm{jet}}, \eta\right), \Delta_{\mathrm{JES}}^{\mathrm{pT}}\left(p_{T}^{\mathrm{jet}}, \eta\right)\right]
$$




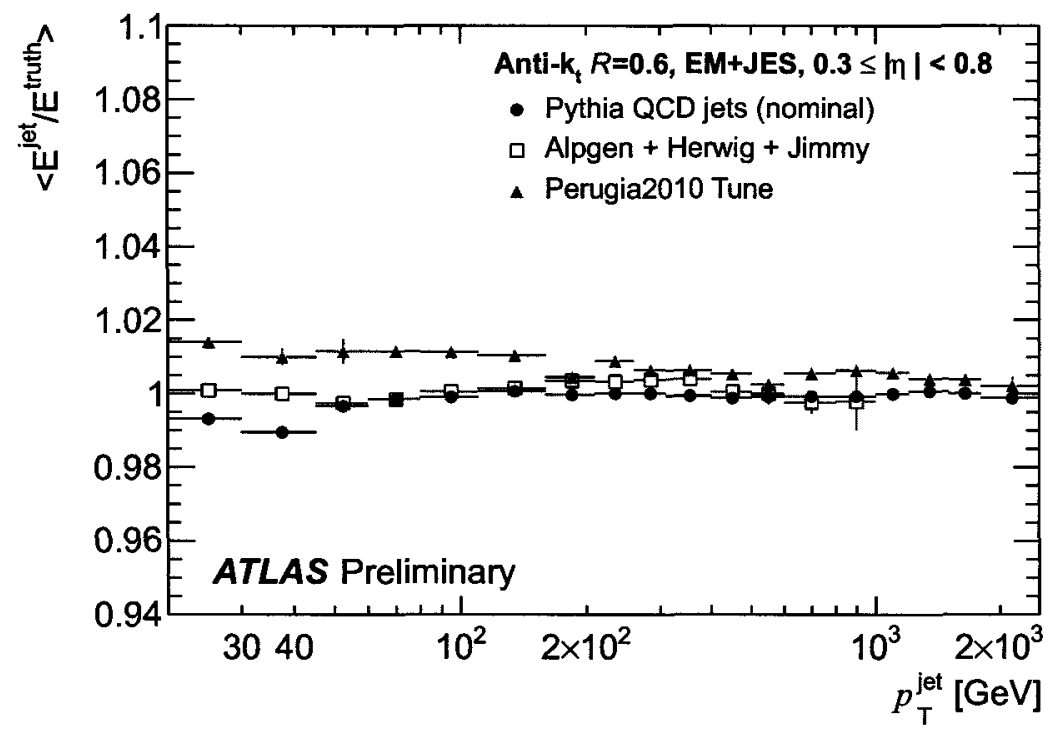

(a)

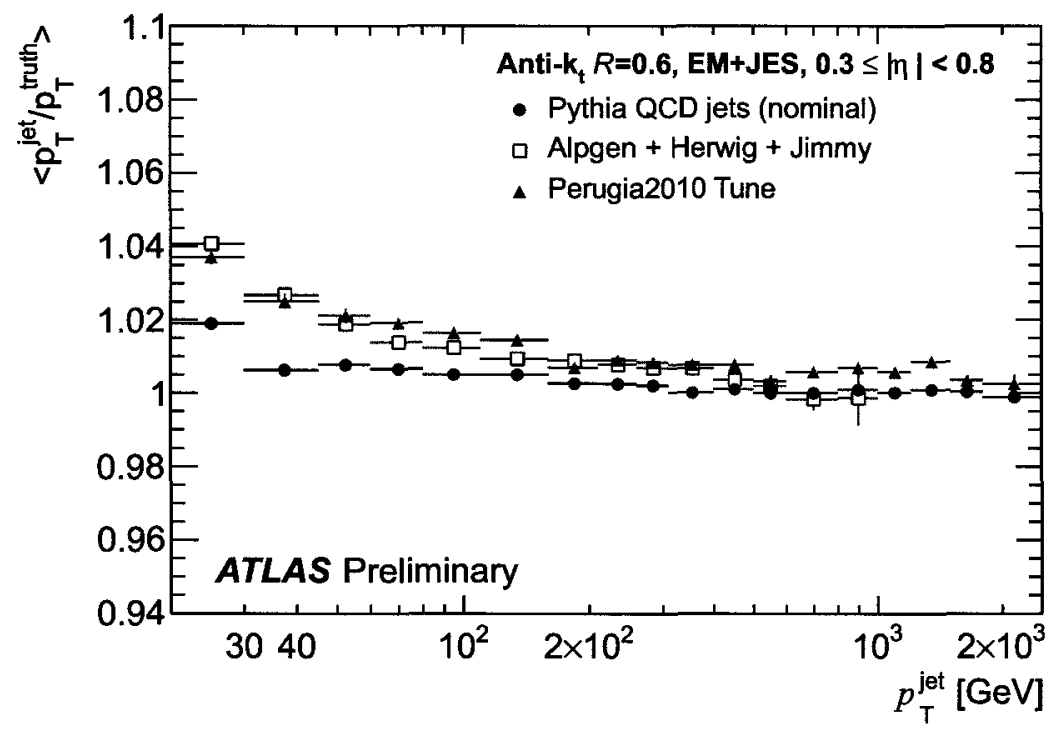

(b)

Figure 7.2: Simulated energy response (a) and $p_{\mathrm{T}}^{\text {jet }}$ response (b) as a function of $p_{\mathrm{T}}^{\text {jet }}$ in the central region $(0.3<|\eta|<0.8)$ for Alpgen+Herwig+Jimmy (open squares) and Pythia with the Perugia2010 tune (full triangles). The response of the nominal Monte Carlo sample is shown for comparison (full circles). 


\subsection{Uncertainties due to Noise Thresholds}

As described in Section 5.2, clusters are constructed based on the signal-to-noise ratio of calorimeter cells, where the noise refers to (the RMS of) the measured cell energy distribution in events with no energy depositions from collision events. Discrepancies between the simulated noise and the real noise in data can lead to differences in the cluster shapes and to the presence of fake clusters, which ultimately affect the jet reconstruction. For data, the noise can change over time ${ }^{1}$, while the noise RMS used in the simulation are fixed at the time of the production of the simulated data. This can lead to differences in the jet reconstruction and calibration if the simulated electronic noise injected in the Monte Carlo simulation does not reflect the noise in data.

The effect of the calorimeter cell noise (mis-)modelling on the jet response is estimated by reconstructing topoclusters, and thereafter jets, in Monte Carlo using the noise RMS measured in data. The actual energy and noise baseline simulated in the MC are left unchanged, but the values of the thresholds used to include a given calorimeter cell in a topocluster are based on the cell noise RMS measured in data. The response of jets reconstructed with the modified noise thresholds are compared with the response of the jets reconstructed in exactly the same sample using the default MC noise thresholds. A series of cell noise thresholds values $(10 \%, 7 \%, 5 \%)$ was used to shift the noise thresholds for all cells [26] to further study the effect on the jet response. Increasing the cell thresholds in MC by $7 \%$ for each cell was found to give a similar shift in the jet response to using the noise RMS from data. Raising

\footnotetext{
${ }^{1}$ Time-dependent noise changes for single cells in data are accounted for with frequent measurements of the level of noise.
} 
and lowering the cell thresholds by $7 \%$ shows that the effect on the jet response from varying the cell noise thresholds is symmetric. This allows to use the calorimeter cell noise thresholds derived from data as a representative sample of the uncertainty on the jet energy scale. This covers both the case when more and less noise is present in data with respect to the simulation.

The maximal observed change in jet response is used to estimate the uncertainty on the jet energy measurement due to the calorimeter cell noise modelling. It is found to be below $3 \%$ for the whole pseudorapidity range, and negligible for jets with transverse momenta above $45 \mathrm{GeV}$. The uncertainties assigned to jets with transverse momenta below $45 \mathrm{GeV}$ are:

- $1 \%$ and $2 \%$ for $20 \mathrm{GeV} \leq p_{\mathrm{T}}^{\text {jet }}<30 \mathrm{GeV}$ for anti- $k_{T}$ jets with distance parameters $R=0.4$ and $R=0.6$, respectively,

- $1 \%$ for $30 \mathrm{GeV} \leq p_{\mathrm{T}}^{\text {jet }}<45 \mathrm{GeV}$.

\subsection{Single Particle Tracking}

Single charged hadrons passing through the ATLAS tracking detector can be used to derive an uncertainty on the energy measurement in the ATLAS calorimeters. The tracking detector provides precise momentum measurements of the hadrons, allowing for direct comparison with the calorimeter energy deposits. The energy-momentum ratio $E / p$ is used as an estimate of the uncertainty for single particle response. At the truth level, this ratio is not generally unity, but is greater than or equal to one, depending on the mass of the particle. This must be taken into account when deriving the uncertainty. Since jets are composed of many hadrons, the uncertainty on the jet 
energy scale can be obtained from the uncertainty of individual particles constituting the jet. A further complication arises since a fraction of jets is electrically neutral, and therefore does not leave tracking information. This can be compensated with knowledge of the average neutral particle fraction of jets, and the average calorimeter response uncertainty for neutral particles.

The $E / p$ measurements have been performed with the ATLAS detector in the pseudorapidity range $|\eta|<0.8$ and particle momentum range $0.5 \mathrm{GeV}<p<20 \mathrm{GeV}$ [27]. The obtained uncertainty is propagated to all the pseudorapidity regions, since the forward regions are calibrated relative to the central. In the central region, the single particle uncertainty is the largest component of the JES uncertainty. This is especially true for particles in jets with large momenta $(p>400 \mathrm{GeV})$, where track curvature and hence momenta are practically unmeasurable.

\subsection{Uncertainties from Material Description}

The jet energy scale is affected by possible deviations in the description of the material in front of and within the calorimeter. In practice the jet energy scale calibration has been derived to restore the energy lost under the assumption of the geometry and material simulated in the nominal Monte Carlo sample. Simulated detector geometries that include systematic variations to the material budget have been designed using test-beam measurements [28], in addition to $900 \mathrm{GeV}$ and $7 \mathrm{TeV}$ data analysis [29, 30, 31, 32]. Specific Monte Carlo samples have been produced using these distorted geometries.

In the case of uncertainties derived with in-situ techniques, such as those coming from the single particle tracking measurements detailed in Section 7.5, most of 
the effects on the jet response due to additional dead material are already taken into account because the measurement is performed directly on the ATLAS detector. However, the quality criteria on the track selection for the single particle tracking measurement effectively only allow particles that have not interacted hadronically in the inner detector to be included in the measurement. Therefore the effect of dead material in the inner detector needs to be taken into account for particles in the momentum range of the single particle tracking measurement. This is achieved using a specific Monte Carlo sample where the material budget is systematically varied adding $5 \%$ of material to the existing inner detector services. The uncertainty derived from the comparison of the distorted material response to the nominal response is then scaled by the fraction of particles within the single particle tracking momentum range. This uncertainty is shown in Figure 7.3.

Electrons, photons and hadrons with momenta $p>20 \mathrm{GeV}$ are not included in the single particle tracking measurements and therefore there is no in-situ estimate on the effect of any additional material in front of the calorimeters. This uncertainty is estimated using a dedicated Monte Carlo simulation sample where the overall detector material is systematically varied within the current uncertainties on the detector geometry knowledge. The overall changes in the detector geometry include:

- the $5 \%$ increase in the inner detector material mentioned above,

- an extra 0.1 radiation length $\left(X_{0}\right)$ has been placed in the cryostat in front of the barrel of the electromagnetic calorimeter $(|\eta|<1.5)$,

- an extra $0.05 X_{0}$ has been placed between the presampler and the first layer of the electromagnetic calorimeter, 
- an extra $0.1 X_{0}$ has been placed in the cryostat after the barrel of the electromagnetic calorimeter,

- extra material has also been placed in the in barrel-endcap transition region in the electromagnetic calorimeter $(1.37<|\eta|<1.52)$.

The uncertainty contribution due to the overall additional detector material is estimated by comparing the calibrated EM+JES jet response in the Monte Carlo sample with the distorted geometry with the nominal jet response (see Figure 7.3), and scaled by the average fraction of electrons, photons and high transverse momentum hadrons within a jet as a function of $p_{T}$.

\subsection{Uncertainty due to Multiple Interactions}

Particles produced by multiple soft proton-proton interactions in the same bunch crossing additional to the event of interest (in-time pile up) can produce additional energy deposits that are reconstructed within the jet. As briefly described in Section 5.5.1, and fully detailed in Ref. [33], an average offset correction is applied to account for the average increase of the jet energy due to pile up. This correction is parameterised as a function of the measured number of primary vertices $N_{\mathrm{PV}}$.

The estimate of the remaining uncertainty on the jet energy scale after applying the pile-up correction is based on the studies described in Section 5 of Ref. [33]. The contributions to the uncertainty are estimated from studies that account for:

- the variation of the average offset-corrected calorimeter jet energy for calorimeter jets matched to track-jets as a function of the number of primary vertices, 


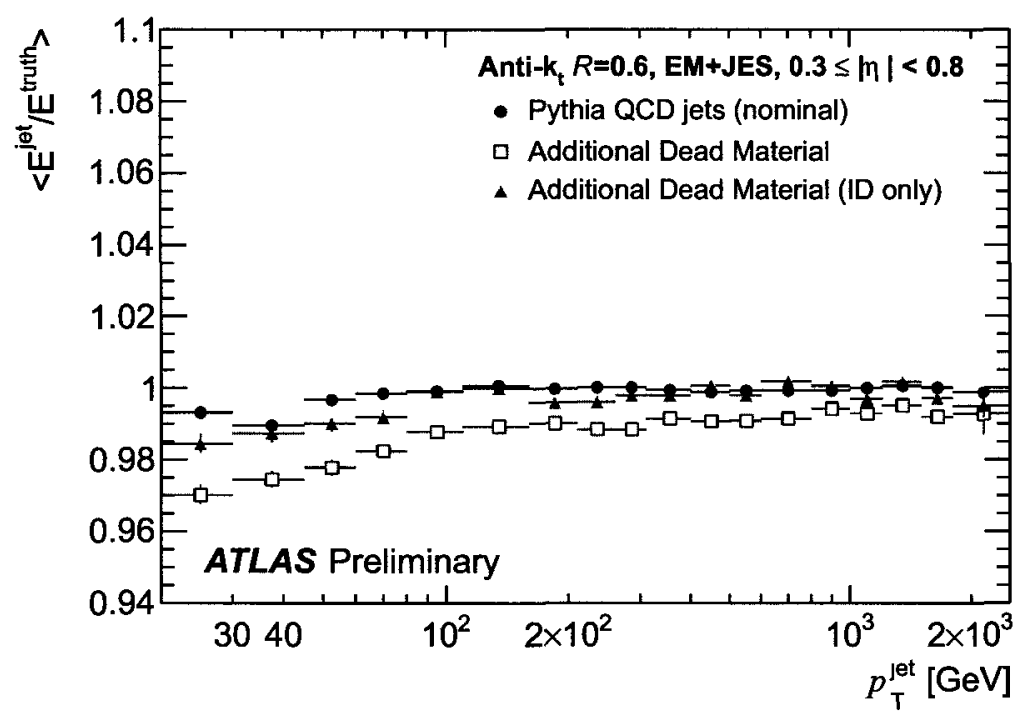

(a)

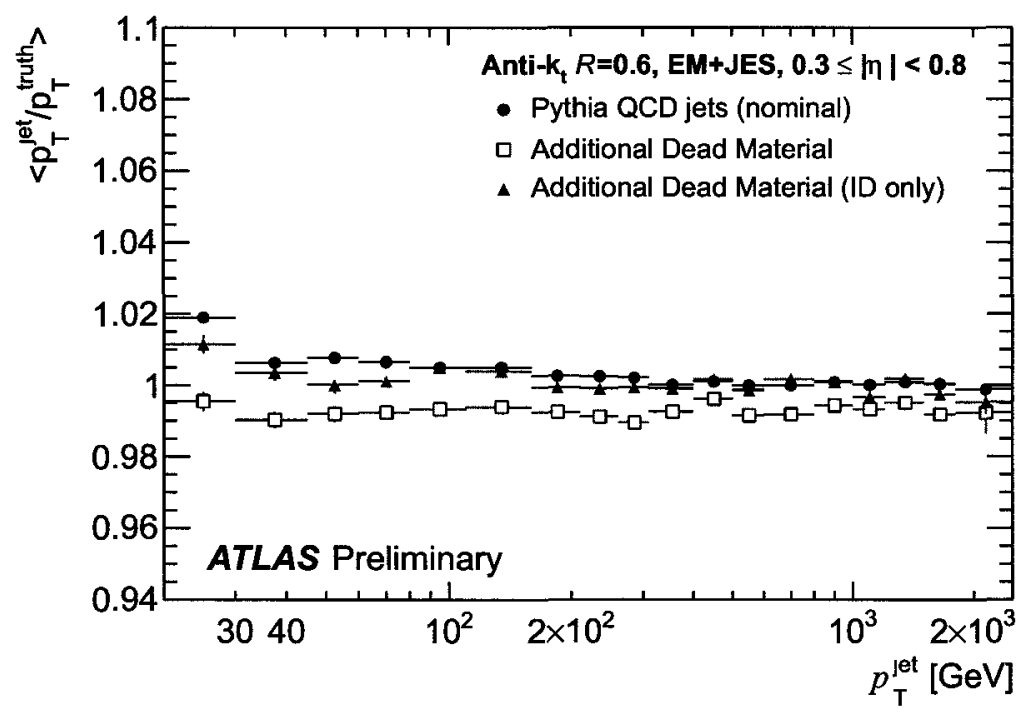

(b)

Figure 7.3: Simulated (a) jet energy response and (b) jet $p_{T}$ response as a function of $p_{\mathrm{T}}^{\text {jet }}$ in the central region $(0.3<|\eta|<0.8)$ in the case of additional dead material in the inner detector (full triangles) and in both the inner detector and the calorimeters (open squares). The response within the nominal Monte Carlo sample is shown for comparison (full circles). 
- the effect of variations of the trigger selection on the measured tower energy distribution that is input to the offset correction,

- the mapping of the tower-based offset correction to a per-jet offset correction,

- the non-closure of the tower-based offset correction as evaluated by the dependence of the corrected calorimeter jet energy for calorimeter jets matched to track-jets as a function of the number of primary vertices.

The uncertainty on the jet energy scale due to pile up is estimated by adding all uncertainties in quadrature. Since the track-jet method can be used only up to $|\eta|<1.9$ due to the limited coverage of the tracking detector, the dijet balance method that is used for the $\eta$ intercalibration uncertainty has been used to estimate the uncertainty for $|\eta|>1.9$ by comparing the relative jet response in events with only one reconstructed vertex with the response measured in events with several reconstructed vertices.

The offset correction and its uncertainty are derived as a function of the number of reconstructed vertices. This allows the correction and its uncertainty to be valid also for data periods where the number of reconstructed primary vertices is higher than the period where the correction is derived.

Figure 7.4 shows the relative uncertainty due to pile up in the case of two measured primary vertices. In this case, the uncertainty due to pile up for central jets with $p_{T}=20 \mathrm{GeV}$ and pseudorapidity $|\eta| \leq 0.8$ is about $1 \%$, while it amounts to about $2 \%$ for jets with pseudorapidity $2.1<|\eta|<2.8$, and to less than $2.5 \%$ for all jets with $|\eta| \leq 4.5$. In the case of three primary vertices, the pile-up uncertainty is approximately twice that of $N_{\mathrm{PV}}=2$, and with four primary vertices the uncertainty 


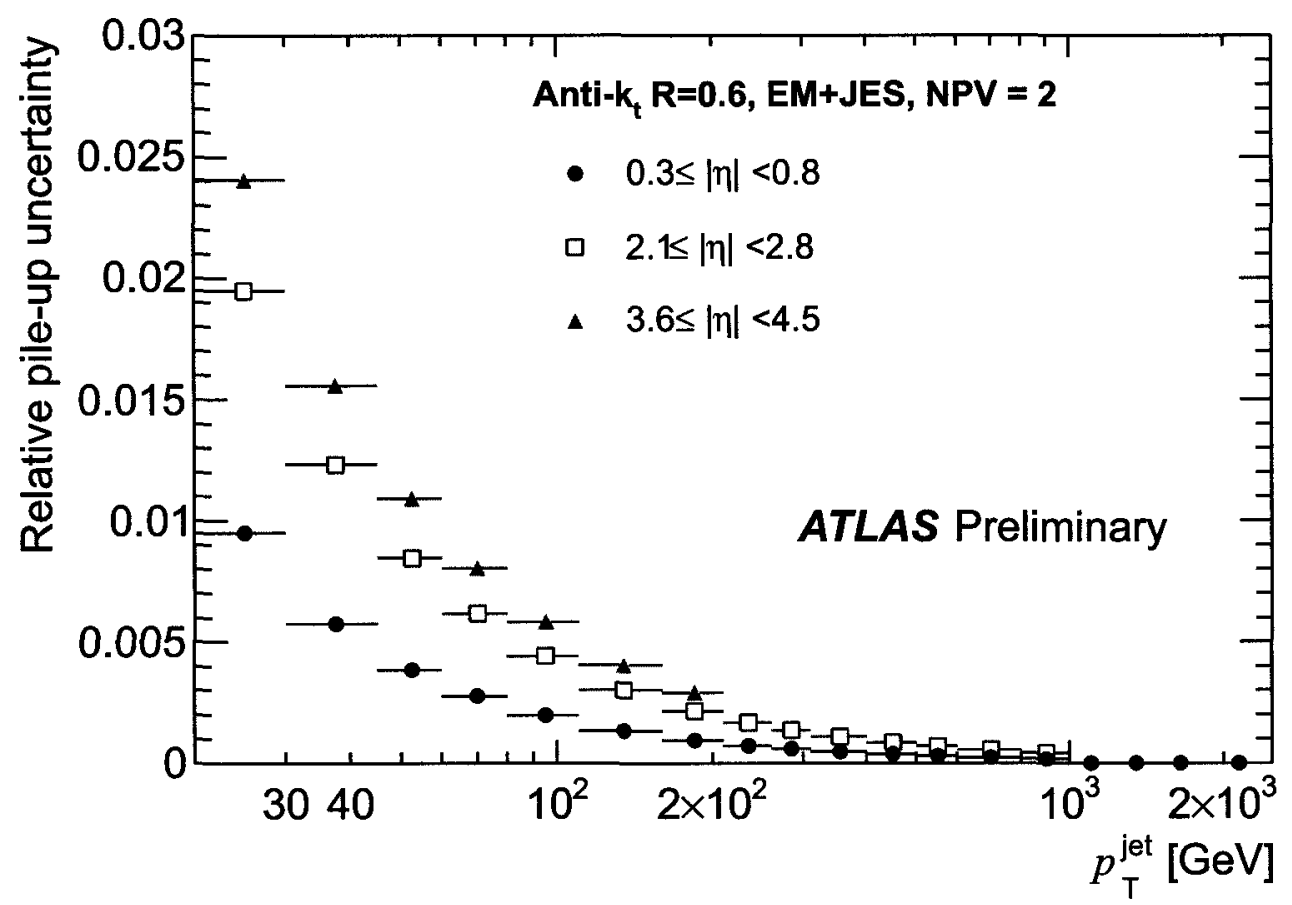

Figure 7.4: Relative pile-up uncertainty for anti- $k_{T}$ jets with $R=0.6$ in the case of two measured primary vertices, $N_{\mathrm{PV}}=2$, for central $(0.3<|\eta|<0.8$, full circles $)$, endcap $(2.1<|\eta| \leq 2.8$, open squares) and forward (3.6< $<\eta \mid \leq 4.5$, full triangles) jets as a function of jet $p_{T}$.

for central, endcap and forward jets is less than $3 \%, 6 \%$, and $8 \%$, respectively. The relative uncertainty due to pile up for events with up to 5 additional interactions becomes less than $1 \%$ for all jets with $p_{T}>200 \mathrm{GeV}$.

The effect of additional proton-proton interactions from different bunch crossings that can be caused by trains of consecutive bunches (out-of-time pile up) has been studied separately. The effect of out-of-time pile up on jet reconstruction has been found to be negligible in the 2010 data-set due to the reduced LHC collision rate compared to the design luminosity. 


\subsection{Total JES Uncertainty}

The total uncertainty on the jet energy scale is obtained by assuming that all the error components are uncorrelated and simply adding them in quadrature. Figures 7.5, 7.6, and 7.7 show the final fractional jet energy scale systematic uncertainty and its individual contributions as a function of jet $p_{\mathrm{T}}^{\text {jet }}$ for three selected $\eta$ regions.

The fractional JES uncertainty in the central region varies roughly from $2.5 \%$ to $4.5 \%$ for $p_{\mathrm{T}}^{\text {jet }}<60 \mathrm{GeV}$, and it is between $2.5 \%$ and $3 \%$ for $60 \mathrm{GeV} \leq p_{\mathrm{T}}^{\text {jet }}<800 \mathrm{GeV}$.

For jets with $p_{\mathrm{T}}^{\text {jet }}>800 \mathrm{GeV}$, the uncertainty goes from $3 \%$ to $4 \%$, due to the larger uncertainties for particles with momentum beyond $400 \mathrm{GeV}$ comprised in these jets. The uncertainty amounts to up to $7 \%$ and $3 \%$, respectively, for $p_{\mathrm{T}}^{\text {jet }}<60 \mathrm{GeV}$ and $p_{\mathrm{T}}^{\text {jet }}>60 \mathrm{GeV}$ in the endcap region, where the central uncertainty is taken as a baseline and the uncertainty due to the relative calibration is added. In the forward region, a $13 \%$ uncertainty is present for $p_{\mathrm{T}}^{\text {jet }}<60 \mathrm{GeV}$ dominated by the intercalibration; the large uncertainty is mostly due to modelling of soft QCD physics and the underlying event in the forward region (see Chapter 6).

Table 7.1 presents a summary of the maximum uncertainties in the different $\eta$ regions for anti- $k_{T}$ jets with distance parameter of $R=0.6$ and with $p_{\mathrm{T}}^{\text {jet }}$ of $20 \mathrm{GeV}$, $200 \mathrm{GeV}$ and $1.5 \mathrm{TeV}$ as an example.

The same study has been repeated for anti- $k_{T}$ jets with distance parameter $R=$ 0.4 , and the estimate of the JES uncertainty is comparable to anti- $k_{T}$ jets with $R=$ 0.6 .

The JES uncertainty for anti- $k_{T}$ jets with $R=0.4$ is between $\approx 4 \%(8 \%, 14 \%)$ at low jet $p_{\mathrm{T}}^{\text {jet }}$ and $\approx 2.5 \%-3 \%(2.5 \%-3.5 \%, 5 \%)$ for jets with $p_{T}>60 \mathrm{GeV}$ in the central (endcap, forward) region, and it is summarised in Table 7.2. 


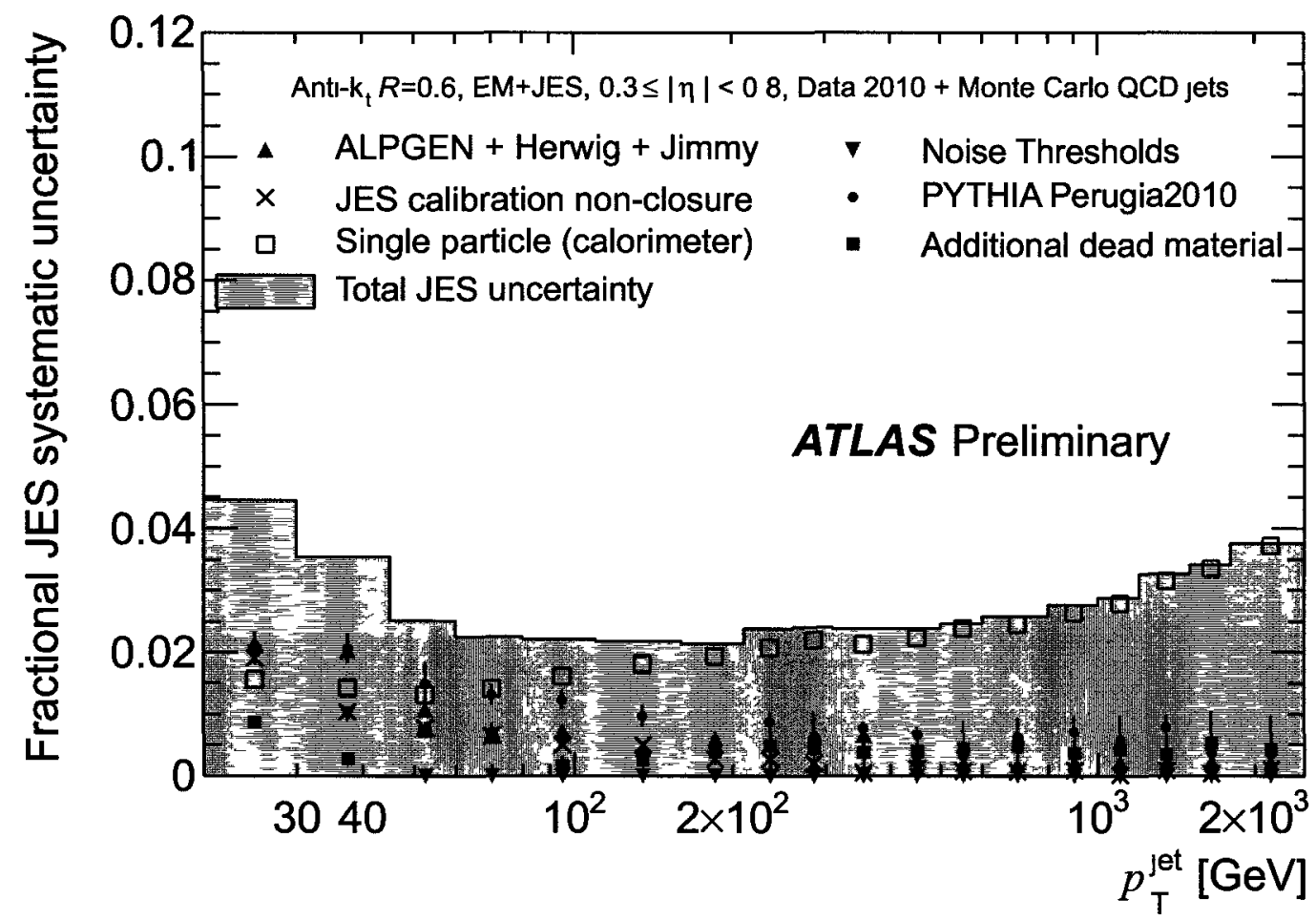

Figure 7.5: Fractional jet energy scale systematic uncertainty as a function of $p_{\mathrm{T}}^{\text {pet }}$ for jets in the pseudorapidity region $0.3 \leq|\eta|<0.8$ in the calorimeter barrel. The total uncertainty is shown as the solid light blue area. The individual sources are also shown, with uncertainties from the fitting procedure if applicable. 


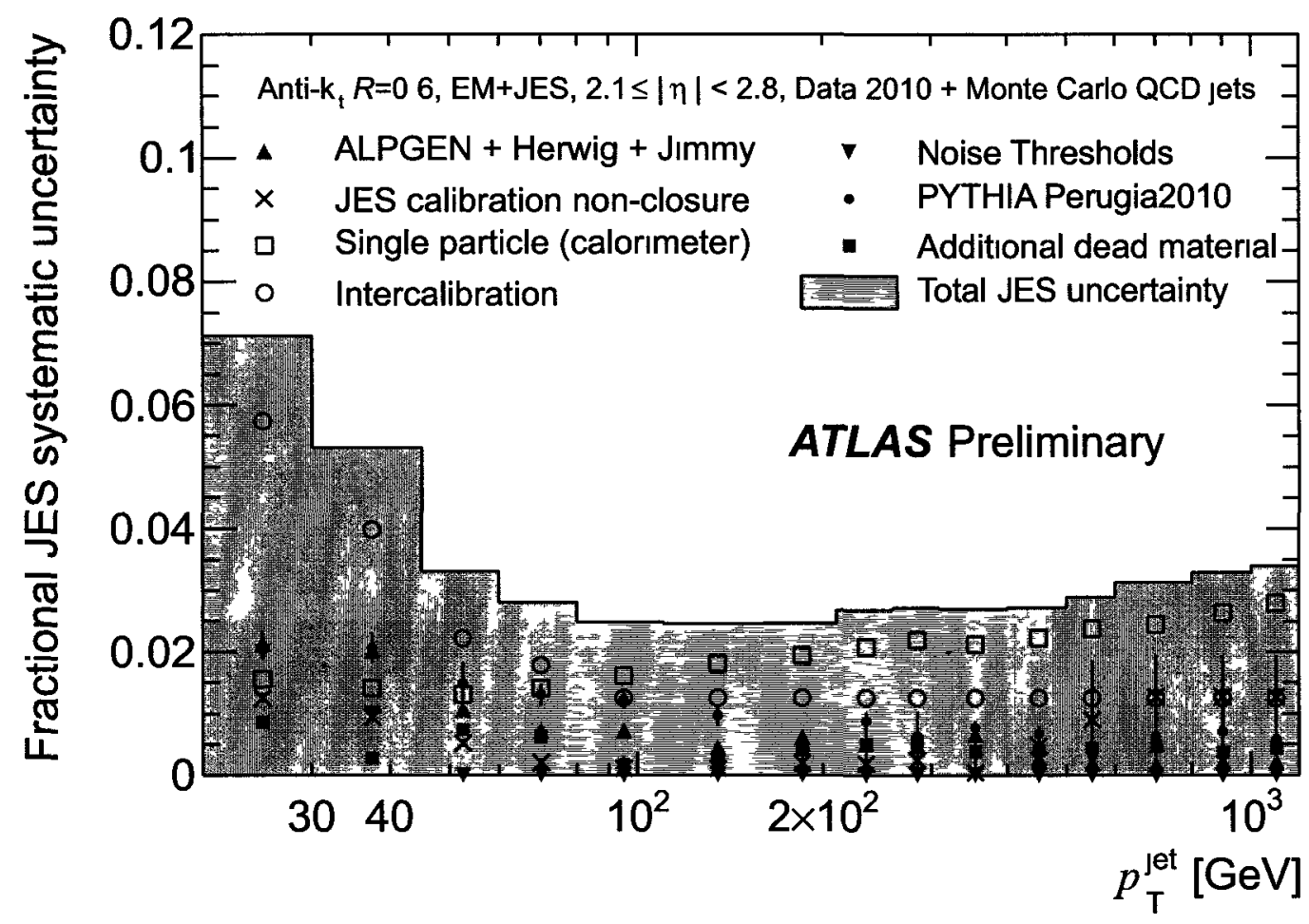

Figure 7.6: Fractional jet energy scale systematic uncertainty as a function of $p_{\mathrm{T}}^{\text {et }}$ for jets in the pseudorapidity region $2.1 \leq|\eta|<2.8$. The JES uncertainty in the endcap region is extrapolated from the barrel uncertainty, with the uncertainty contribution from the $\eta$ intercalibration between central and endcap jets in data and Monte Carlo added in quadrature. The total uncertainty is shown as the solid light blue area. The individual sources are also shown, with uncertainties from the fittmg procedure if applicable. 


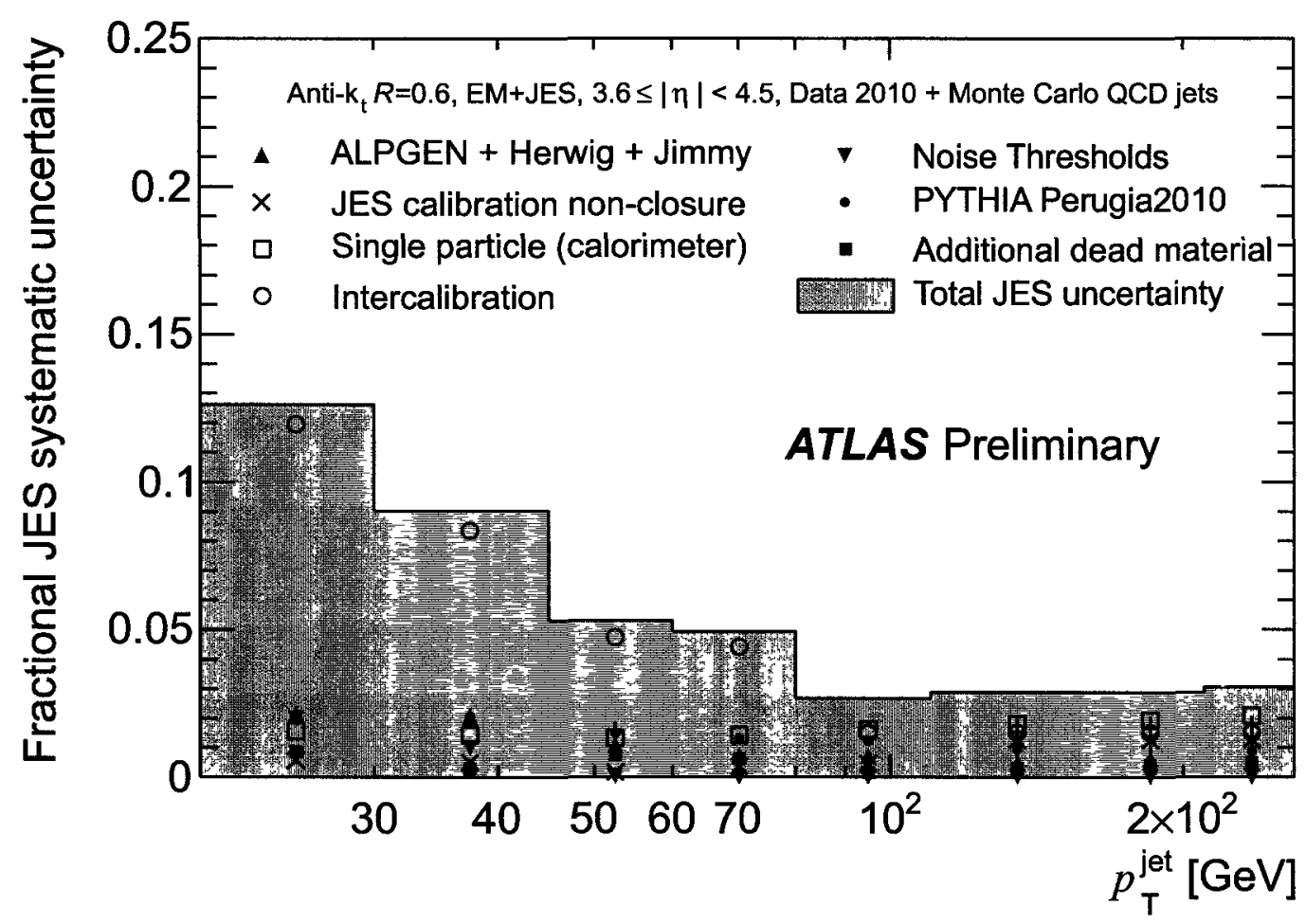

Figure 7.7: Fractional jet energy scale systematic uncertainty as a function of $p_{\mathrm{T}}^{\text {jet }}$ for jets in the pseudorapidity region $3.6<|\eta|<4.5$. The JES uncertainty for the forward region is extrapolated from the barrel uncertainty, with the uncertainty contribution from the $\eta$ intercalibration between central and forward jets in data and Monte Carlo added in quadrature. The total uncertainty is shown as the solid light blue area. The individual sources are also shown, with uncertainties from the fitting procedure if applicable. 
Table 7.1: Summary of the maximum EM+JES jet energy scale systematic uncertainties for different $p_{\mathrm{T}}^{\mathrm{jet}}$ and $\eta$ regions from Monte Carlo-based study for anti- $k_{T}$ jets with $R=0.6$.

\begin{tabular}{|c|c|c|c|}
\hline$\eta$ region & \multicolumn{3}{|c|}{ Maximum fractional JES Uncertainty } \\
\hline & $p_{T}^{\text {jet }}=\mathbf{2 0} \mathbf{~ G e V}$ & $p_{T}^{\text {jet }}=\mathbf{2 0 0} \mathbf{~ G e V}$ & $p_{T}^{\text {jet }}=\mathbf{1 . 5} \mathbf{~ T e V}$ \\
\hline $0<|\eta|<0.3$ & $4.6 \%$ & $2.3 \%$ & $3.1 \%$ \\
$0.3<|\eta|<0.8$ & $4.5 \%$ & $2.2 \%$ & $3.3 \%$ \\
$0.8<|\eta|<1.2$ & $4.5 \%$ & $2.4 \%$ & $3.4 \%$ \\
$1.2<|\eta|<2.1$ & $5.5 \%$ & $2.5 \%$ & $3.5 \%$ \\
$2.1<|\eta|<2.8$ & $7.1 \%$ & $2.5 \%$ & \\
$2.8<|\eta|<3.2$ & $8.5 \%$ & $3.0 \%$ & \\
$3.2<|\eta|<3.6$ & $8.7 \%$ & $3.0 \%$ & \\
$3.6<|\eta|<4.5$ & $12.6 \%$ & $2.9 \%$ & \\
\hline
\end{tabular}

Table 7.2: Summary of the maximum EM+JES jet energy scale systematic uncertainties for different $p_{\mathrm{T}}^{\text {jet }}$ and $\eta$ regions from Monte Carlo based study for anti- $k_{T}$ jets with $R=0.4$.

\begin{tabular}{|c|c|c|c|}
\hline$\eta$ region & \multicolumn{3}{|c|}{ Maximum fractional JES Uncertainty } \\
\hline & $p_{T}^{\text {jet }}=\mathbf{2 0 ~} \mathbf{~ G e V}$ & $p_{T}^{\text {jet }}=\mathbf{2 0 0} \mathbf{~ G e V}$ & $p_{T}^{\text {jet }}=\mathbf{1 . 5} \mathbf{~ T e V}$ \\
\hline $0<|\eta|<0.3$ & $4.1 \%$ & $2.3 \%$ & $3.1 \%$ \\
$0.3<|\eta|<0.8$ & $4.3 \%$ & $2.4 \%$ & $3.3 \%$ \\
$0.8<|\eta|<1.2$ & $4.3 \%$ & $2.5 \%$ & $3.5 \%$ \\
$1.2<|\eta|<2.1$ & $5.2 \%$ & $2.6 \%$ & $3.6 \%$ \\
$2.1<|\eta|<2.8$ & $8.2 \%$ & $2.9 \%$ & \\
$2.8<|\eta|<3.2$ & $10.1 \%$ & $3.5 \%$ & \\
$3.2<|\eta|<3.6$ & $10.3 \%$ & $3.7 \%$ & \\
$3.6<|\eta|<4.5$ & $13.8 \%$ & $5.3 \%$ & \\
\hline
\end{tabular}




\section{Chapter 8}

\section{Inclusive Jet and Dijet Cross}

\section{Section Measurements}

\subsection{Introduction}

At the LHC, jet production is the dominant high $p_{T}$ process and as such gives the first glimpse of physics at the $\mathrm{TeV}$ scale. The study of jet production is therefore an integral first step of the LHC program.

Jet cross sections and properties are key observables in high-energy physics experiments. They have been measured in $e^{+} e^{-}, e p, p \bar{p}$, and $p p$ colliders, as well as in $\gamma p$ collisions. They have provided precise measurements of the properties of the strong interaction, have been used to obtain information about the structure of the proton and the photon [34], and have become important tools for searching for physics beyond the Standard Model.

This chapter presents the measurement of the inclusive jet and dijet cross sections in p-p collisions at a center-of-mass (CM) energy of $7 \mathrm{TeV}$ using the ATLAS 
detector at the LHC. The probability to produce jets is quantified by a measurement of the cross section in units of pico-barns $\left(\mathrm{pb}=10^{-12} \mathrm{~b}\right.$ ), where $1 \mathrm{~b}=10^{-24} \mathrm{~cm}^{2}$. The measurements were performed using the full 2010 dataset, consisting of a total integrated luminosity of $37.3 \pm 1.2 \mathrm{pb}^{-1}$. The LHC's unprecedented collision energy and the ATLAS detector's wide pseudorapidity coverage, made possible by the FCal, probe QCD in a new, largely unexplored region of phase space. Comparisons are made to Next-to-Leading Order (NLO) perturbative QCD (pQCD) corrected for non-perturbative effects. In addition to a validation of the theory in a new kinematic regime, the data also provide sensitivity to parton distribution functions in a region where they are currently poorly constrained.

\subsection{Cross Section Definition}

The number of events observed in a collider experiment is directly proportional to the integrated luminosity, $\mathcal{L}$, the event cross section, $\sigma$, and the event detection efficiency $\epsilon$ :

$$
N_{\text {events }}=\mathcal{L} \sigma \epsilon,
$$

where $\mathcal{L}$ is the time integral of the instantaneous machine luminosity $L$ :

$$
\mathcal{L}=\int L d t
$$

The instantaneous machine luminosity can be regarded as the total instantaneous flux of protons, i.e. the total incident number of protons per unit area per unit time. Knowing the integrated luminosity and the detection efficiency $(0<\epsilon<1)$, the event 
cross section can be measured by recording the number of detected events. In this context, an event is an occurrence of a particular process, not necessarily a single p-p interaction.

The inclusive jet cross sections are presented as double differential functions of jet

$p_{T}$ and $y,\left(\frac{d^{2} \sigma}{d p_{T} d y}\right)$. Inclusive implies that all jets observed are counted, and a cross section is calculated from the observed number of jets, $N_{\text {jets }}$, by rearranging Equation (8.1). For this measurement then, each jet is an occurrence, i.e. each jet's $p_{T}$ and $y$ are an entry in the $\frac{d^{2} \sigma}{d p_{T} d y}$ distribution. The inclusive jet measurement spans the phase space region $20 \mathrm{GeV}<p_{T}<1500 \mathrm{GeV}$, and $|y|<4.4$. In principle, the jet $p_{T}$ can reach the kinematic limit of half the $\mathrm{CM}$ energy, $3500 \mathrm{GeV}$, but this is highly improbable and no such jets are observed in the current dataset.

The dijet cross sections are presented as double differential functions of the dijet invariant mass, $m_{12}$, binned in the maximum rapidity of the two leading jets, $|y|_{\max }$ $=\max \left(\left|y_{1}\right|,\left|y_{2}\right|\right)$. The invariant mass is defined as (using the natural unit system)

$$
m_{12}^{2}=\left(p_{1}+p_{2}\right)^{2}=\left[\left(E_{1}+E_{2}\right)^{2}-\left({\overrightarrow{p_{1}}}^{2}+{\overrightarrow{p_{2}}}^{2}\right)\right]^{2},
$$

where $p_{\imath}, E_{\imath}$, and $\vec{p}_{\imath}$ are the 4 -vector momentum, energy, and 3-vector momentum of the $i$ th jet, respectively. Each dijet pair within a p-p interaction is an occurrence; the dijet system's $m_{12}$ and $|y|_{\max }$ are entries in the $\frac{d^{2} \sigma}{d m_{12} d|y|_{\max }}$ distribution. The dijet mass spectra are studied in the intervals $60 \mathrm{GeV}<m_{12}<4.1 \mathrm{TeV}$, and $|y|_{\max }<2.8$. For this measurement the $|y|_{\max }$ is limited to 2.8 due to the complexity of triggering dijet events outside this acceptance using a combination of central and forward jet triggers. 


\subsection{Theoretical Predictions}

Theoretical calculations for jet production arising from p-p collisions embody three different important aspects of QCD: PDFs, perturbative calculations, and hadronization + underlying event (a non-perturbative calculation).

Since p-p collisions are the result of interactions between individual partons, the parton distribution functions (PDFs) are required for the calculation. The PDFs, however, are not calculable by QCD but are instead obtained from experimental data. For the theoretical calculations carried out in this analysis, several different PDF sets, obtained from previous experiments, are used.

Once the PDFs have been used to select particular partons to take part in the interaction, perturbative QCD calculations are carried out. This corresponds to the hard scatter of the event. In this analysis the calculations are carried out at nextto-leading order (NLO). The ability to perform a perturbative calculation, which is essentially a series expansion in the interaction coupling strength, relies heavily on the strength of the interaction. The strong coupling strength constant, $\alpha_{s}$, must be smaller than one for the expansion to converge. The coupling strength depends on the momentum transfer between the partons and at the high energies of the LHC the coupling constant is smaller than one, allowing perturbative calculations to be carried out.

The final state of the hard scatter calculation contains two outgoing partons which then hadronize into jets. In addition, the activity that arises from the underlying event must also be taken into account for meaningful comparison to data. These comprise the third part of the calculation: non-perturbative corrections which take the cross section calculation from outgoing partons to outgoing jets. Figure 8.1 shows 
a schematic of the steps involved in a theoretical calculation.

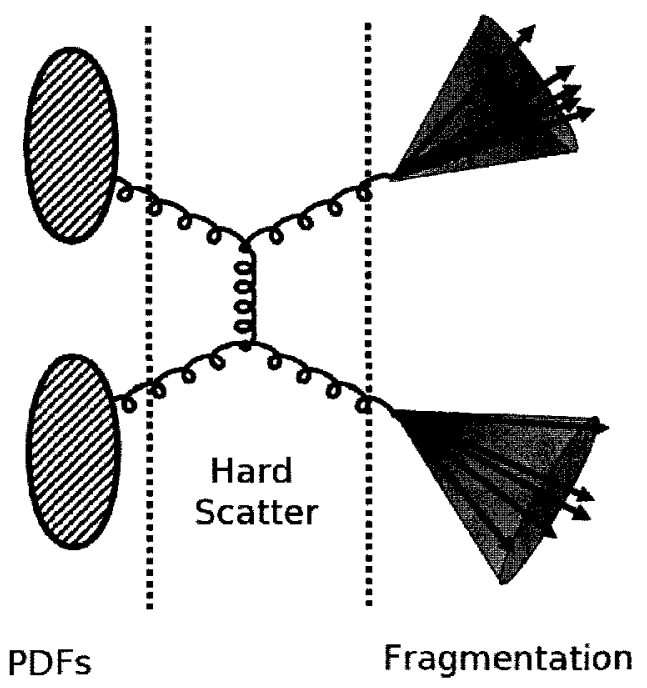

Figure 8.1: The three stages of the QCD calculation process. PDFs are needed to select specific partons within the protons to take part in the hard scatter. The hard scatter involves gluon exchange between two partons and is a perturbative calculation. The last step hadronizes the partons such that the calculation is valid for final state jets. Image adapted from T.J. LeCompte, ATLAS Collaboration.

Two different approaches can be taken to calculate the cross sections. These are discussed in the following two sections.

\subsubsection{Fixed Order NLO pQCD + Non-Perturbative Correc- tions}

The measured jet cross sections are compared to NLO pQCD predictions, with corrections for non-perturbative effects (underlying event and hadronization) applied. The NLOJET++ 4.1.2 [35] program along with the CTEQ6.6 [23] NLO PDFs is used for all baseline calculations. Results were cross-checked with predictions obtained using the CT10 [36], MSTW 2008 [37], NNPDF 2.1 [38], HERA 1.5 [39], and GJR08 [40] 
PDF sets.

Key uncertainties on the NLO prediction come from the uncertainties on the PDF central value, the choice of factorization and renormalization scales, and the uncertainty in the value of the strong coupling constant $\alpha_{s}$. In all cases, the total uncertainty on the theory is defined to be the quadratic sum of the individual uncertainties.

All modern PDF sets have a central member, corresponding to the best estimate of the parton density in the global fit, and a set of error PDF members used for the evaluation of uncertainty associated with this prediction. The PDF uncertainty is of the order of $\sim 3 \%$ at low $p_{T}$, but it increases with transverse momentum and becomes the dominant theoretical uncertainty for jets with $p_{T}$ greater than roughly $200 \mathrm{GeV}$ in the central rapidity region. The CTEQ6.6 PDF uncertainty in the cross section calculation is more than $10 \%$ for jet $p_{T} \geq 1 \mathrm{TeV}[2]$.

The default renormalisation and factorisation scales ( $\mu_{R}$ and $\mu_{F}$, respectively) for both the inclusive jet and the dijet observables are defined to be the $p_{T}$ of the hardest jet in the event. To estimate the uncertainty on the NLO prediction due to neglected higher-order terms, each observable was calculated while varying the renormalisation scale by a factor of two with respect to the default choice. Similarly to estimate the sensitivity to the choice of scale where the PDF evolution is separated from the matrix element, the factorisation scale was separately varied by a factor of two. Thus the total set of renormalisation and factorisation scales used is [2]:

$$
\left(\frac{\mu_{R}}{\mu_{R}^{\text {default }}}, \frac{\mu_{F}}{\mu_{F}^{\text {default }}}\right)=\left(\frac{1}{2}, 1\right),(2,1),\left(1, \frac{1}{2}\right),(1,2),\left(\frac{1}{2}, \frac{1}{2}\right),(2,2) .
$$

The envelope of the variation in the observable is taken as the uncertainty due to 
scale choice.

The uncertainty on the cross section prediction due to the uncertainty on the value of the strong coupling constant, $\alpha_{s}$, is evaluated following the recommendation of the CTEQ group [23]. The uncertainty is taken as one half of the difference between the cross sections with two extreme values of $\alpha_{s}$ :

$$
\Delta \sigma_{\alpha_{s}}=\frac{1}{2}\left|\sigma\left(\alpha_{s}^{+}\right)-\sigma\left(\alpha_{s}^{-}\right)\right|
$$

Each PDF set uses its own range of values for $\alpha_{s}: \alpha_{s}^{0}=0.118, \alpha_{s}^{-}=0.116$ and $\alpha_{s}^{+}=0.120$ for CTEQ6.6; $\alpha_{s}^{0}=0.12, \alpha_{s}^{-}=0.116$ and $\alpha_{s}^{+}=0.123$ for MSTW; $\alpha_{s}^{0}=0.1176, \alpha_{s}^{-}=0.1156$ and $\alpha_{s}^{+}=0.1196$ for HERA; and $\alpha_{s}^{0}=0.119, \alpha_{s}^{-}=0.117$ and $\alpha_{s}^{+}=0.121$ for NNPDF.

This uncertainty hovers between $4 \%$ and $5 \%$ almost flat in jet transverse momentum, but it goes down to $2 \%$ to $3 \%$ for low $p_{T}$ jets [2].

The NLO calculations predict parton-level cross sections, which must be corrected for non-perturbative effects for meaningful comparison with data. This is done by using parton shower generators to evaluate the cross sections with and without hadronization and underlying event contributions. The ratio of the cross section with these contributions to the cross section without them defines a correction factor that is applied, bin-by-bin, to the predicted NLO cross section.

The uncertainty is estimated as the maximum spread of the correction factors obtained from Pythia using the Perugia0, Perugia 2010, and PerugiaX tunes [41, 42] as well as those obtained from Herwig++ [20].

The correction factors are less than unity for $R=0.4$ jets, rising from 0.8 to 1 as a function of $p_{T}$. For $R=0.6$ jets however, the factors are greater than unity, 
decreasing from about 1.4 to 1 as a function of $p_{T}$. This is explained in the following.

The effect of hadronization is to spread energy outside of the jet area, such that hadronization corrections are roughly $0.6(0.8)$ for jet size $R=0.4(0.6)$ at $p_{T} \approx 20$ $\mathrm{GeV}$. This correction slowly increases to $\approx 1$ with increasing $p_{T}$, since the high $p_{T}$ jet originates from the boosted parton while daughter hadrons will have small transverse momentum with respect to the original parton direction.

On the other hand, the underlying event adds extra particles to the jet definition and results in the increase of jet $p_{T}$. The correction to the jet spectrum due to the underlying event activity is roughly $1.2(1.6)$ for jet size $R=0.4(0.6)$ at $p_{T} \approx 20 \mathrm{GeV}$, decreasing to $\sim 1$ as a function of $p_{T}$. This is due to the fact that the small energy density of the underlying event is not correlated with jet $p_{T}$ to the first approximation.

The result of combining the corrections for hadronization and the underlying event, has the outcome mentioned above; $R=0.4$ jets get a correction smaller than unity while the correction is greater than 1 for $R=0.6$ jets. Evidently, the correction for the smaller jets is dominated by the effect of hadronization spreading energy outside of the jet area. The correction for the larger jets is dominated by the underlying event energy falling inside the jet area. Comparisons of the measurements with two jet radii will provide additional information on the correct non-perturbative parameters for jet production at $\sqrt{s}=7 \mathrm{TeV}$.

\subsubsection{NLO ME + Parton Shower}

Different methods to construct event generators that are accurate to NLO are available $[43,44,45]$ for a considerable number of hadron collider processes. The use of an event generator with NLO matrix elements (ME), coherently including the simulation 
of the parton shower, the hadronization, and the underlying event, creates a more coherent theoretical prediction and overcomes the need for separate non-perturbative corrections.

An NLO parton shower Monte Carlo prediction for inclusive jet and dijet production has only recently become available for the first time through the Powheg formalism [46]. Powheg, which uses the Powheg Box package [47], allows one to use either Pythia or Herwig [24] to shower the partons, hadronize them, and model the underlying event. The ATLAS Minimum Bias Tune 1 (AMBT1) [17] and ATLAS Underlying Event Tune 1 (AUET1) [48] are used in Pythia and Herwig, respectively, to model the non-perturbative effects.

In the Powheg algorithm, each event is built by first producing a QCD 2 $\rightarrow 2(3)$ partonic scattering. The renormalisation and factorisation scales are set to be equal to the transverse momentum of the outgoing partons, before proceeding to generate the hardest partonic emission in the event. The MSTW 2008 NLO PDF [37] is used in this step of the simulation. Once the hardest partonic emission is simulated, the events can be evolved to the hadron level by parton-shower event generators.

\subsection{Data Selection}

\subsubsection{Dataset}

The jet cross section measurements detailed in this thesis utilize the full 2010 dataset of proton-proton collisions at $\sqrt{s}=7 \mathrm{TeV}$, with the exceptions of the low- $p_{T}$ region $20 \leq p_{T}<60 \mathrm{GeV}$, and the forward region $|y|>2.8$ for the dijet analysis. For low- $p_{T}$ jets, only data runs taken up to the beginning of June 2010 are considered 
since the instantaneous luminosity of the accelerator was low enough such that pileup contributions were negligible, and the majority of the bandwidth was allocated to the Minimum Bias trigger that is used to collect low- $p_{T}$ events. Figure 8.2 shows the integrated luminosity for each week of the year.

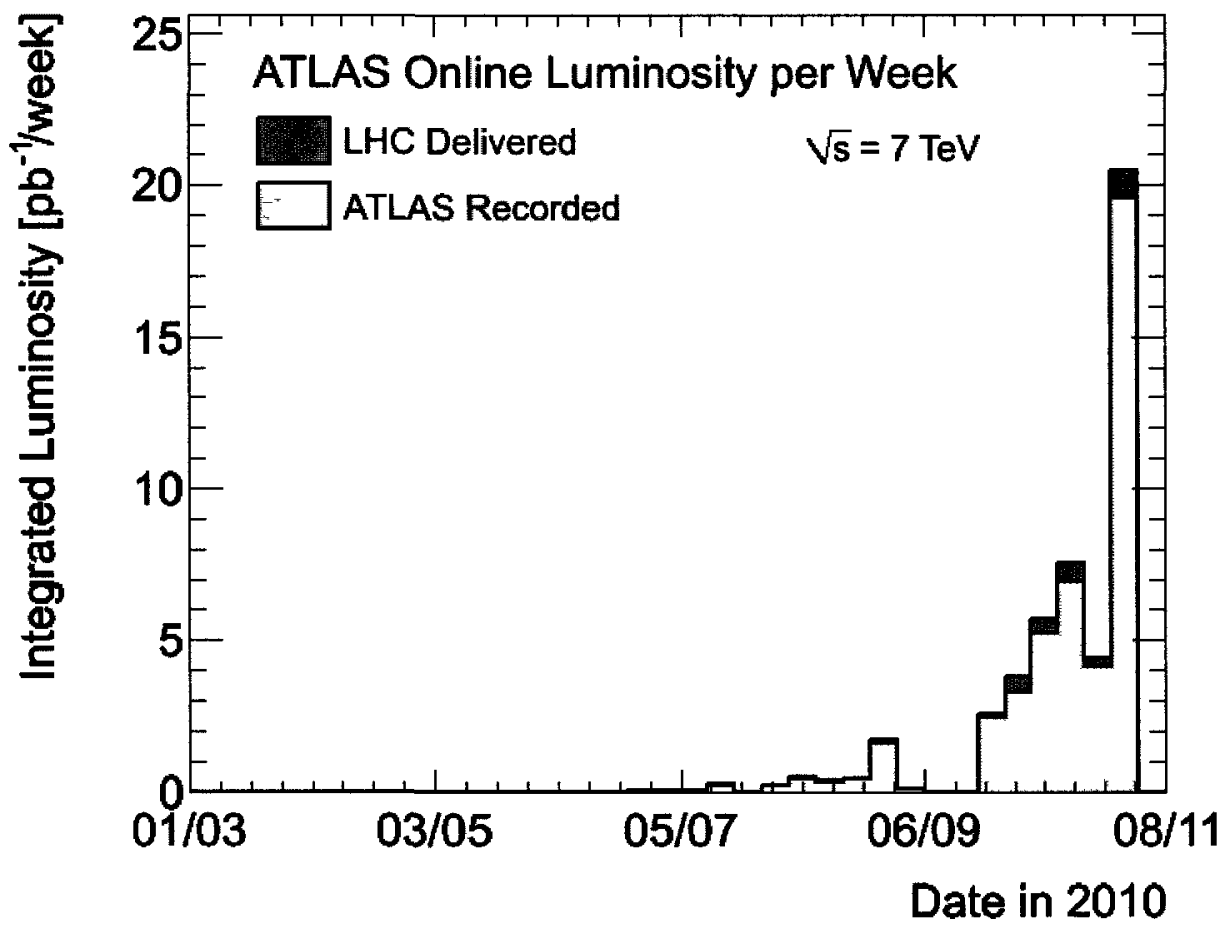

Figure 8.2: Integrated luminosity versus week. Plot taken from the ATLAS public results page: http://atlas.web.cern.ch/Atlas/Collaboration/.

For forward jets in $|y|>2.8$, the first data taking period was not used because the forward jet trigger was not yet commissioned. All data events considered in this analysis require good detector status for the L1 central trigger processor, solenoid magnet, inner detectors (Pixel, SCT, and TRT), calorimeters (barrel, endcap, and forward), luminosity monitor, as well as tracking, jet, and missing energy reconstruction performance. In addition, good data quality was required for the high-level trigger during 
the periods when this device was used for rejection.

\subsubsection{Trigger}

Three different trigger systems have been used in this measurement: the MBTS_1, the central jet trigger, both at L1 and L2 for various thresholds, and the forward jet trigger, also at L1 and L2 for various thresholds.

The MBTS_1 trigger is the primary system to select minimum-bias events in ATLAS. It is based on the Level-1 trigger signal generated by charged particles crossing one of the counters of the Minimum-Bias Trigger Scintillators. It has been demonstrated [49] that due to additional soft radiation away from the main jets, this trigger can be considered fully efficient for events with hard jets pointing in any detector region, i.e. not necessarily inside the acceptance of the counters. In this measurement, the MBTS trigger has been used to select events with low transverse momentum jets, well below the region of full efficiency of the calorimeter-based jet trigger:

The jet trigger is composed of three levels, but its start-up during the 2010 run was gradual. During the early periods when the instantaneous luminosity was sufficiently low, only Level-1 was used in rejection mode, while the other levels were running but only used for validation. In the later periods of the run as the luminosity gradually increased, Level-2 began to be used for rejection. The jet Event Filter was never used to reject events in 2010 .

The cross section is measured bin-by-bin, in bins of $p_{T}-y$ for the inclusive jet analysis and $m_{12}-|y|_{\max }$ for the dijet analysis. Each bin has a specific trigger associated with it that collects and counts the events. The trigger is required to be fully (more than 99\%) efficient across the entire range of the bin. In general, triggers 
are the main source of variation of the detection efficiency, $\epsilon$ in Equation (8.1). The detection efficiency can be quite small in certain bins due to large trigger prescales. This is particularly true for low $p_{T}$ threshold triggers.

For most bins it is trivial to define a trigger efficiency and assign a trigger. In the HEC-FCal transition region, a complication arises due to the splitting of the jet triggers. Central jet triggers span $|\eta|<2.8$ and forward jet triggers span $3.2<|\eta|<$ 4.9. One of the chosen rapidity bins for the inclusive analysis is $2.8<|y|<3.6$, covering the transition region, that has no trigger system present. Neither the central nor the forward jet trigger is fully efficient in this bin. However, the combination of the two, their logical OR, is fully efficient. The central and forward triggers can be combined to measure the cross section in this bin, as described in the following.

Generally the prescale of a trigger is not constant, but varies as the run conditions change, such as the instantaneous luminosity. Luminosity Blocks (LB) are small time periods of a run where beam conditions are stable and trigger prescales are constant.

The integrated luminosity for jets selected by the central trigger is

$$
\mathcal{L}_{\mathrm{J}}=\sum_{\mathrm{LB}} \frac{\mathcal{L}_{\mathrm{LB}}}{P_{\mathrm{LB}}^{\mathrm{J}}},
$$

where $P_{\mathrm{LB}}^{\mathrm{J}}$ is the prescale of the central trigger for each luminosity block, and $\mathcal{L}_{\mathrm{LB}}$ its luminosity.

Similarly, for jets selected by the forward trigger the equivalent luminosity will be

$$
\mathcal{L}_{\mathrm{FJ}}=\sum_{\mathrm{LB}} \frac{\mathcal{L}_{\mathrm{LB}}}{P_{\mathrm{LB}}^{\mathrm{FJ}}} .
$$

Finally, for events taken, before prescale, by both central and forward trigger, the 
equivalent luminosity is $[2]$

$$
\mathcal{L}_{\mathrm{JFJ}}=\sum_{\mathrm{LB}} \frac{\mathcal{L}_{\mathrm{LB}}}{P_{\mathrm{LB}}^{\mathrm{J}} P_{\mathrm{LB}}^{\mathrm{FJ}} /\left(P_{\mathrm{LB}}^{\mathrm{J}}+P_{\mathrm{LB}}^{\mathrm{FJ}}-1\right)} .
$$

If $N_{\mathrm{J}}, N_{\mathrm{FJ}}$ and $N_{\mathrm{JFJ}}$ are used to denote the number of events taken by the central trigger only, by the forward trigger only, and by both, respectively, the cross section, before any other correction, will be given by [2]

$$
\sigma=\frac{N_{\mathrm{J}}}{\mathcal{L}_{\mathrm{J}}}+\frac{N_{\mathrm{FJ}}}{\mathcal{L}_{\mathrm{FJ}}}+\frac{N_{\mathrm{JFJ}}}{\mathcal{L}_{\mathrm{JFJ}}}
$$

ensuring that events passing two triggers are properly treated in a separate category and not double-counted.

\subsubsection{Background Studies}

Background contributions from non-proton-proton collision sources were evaluated by investigating events from trigger streams in which no real collision candidates are expected. In particular, events from the CosmicCalo trigger stream and events in the L1Calo and MinBias trigger stream that pass the trigger requirements for L1_J5_Unpaired, which corresponds to unpaired proton bunches, or L1_J5_empty, which corresponds to empty bunch crossings, were used. A sample of the full dataset consisting of about $2 \mathrm{pb}^{-1}$ was investigated. In this data sample, only one jet that satisfies the event and jet selection criteria was found. Given this, the background rates from non-proton-proton collisions across the entire data period are taken to be negligible. 


\subsubsection{Effects of the Vertex Position}

In a given data event, multiple interactions can occur. Each interaction has its own primary vertex which has its own position along the z-axis (beam line). All jets in the event, however, are made to point back to a single primary vertex, the one with the highest sum of transverse momentum originating from it. To determine systematic errors due to the incorrect modelling of the event vertex position, jet spectra obtained for different $\mathrm{z}$ positions of the first primary vertex were studied. As for the standard analysis, the vertex is required to have at least 5 tracks, and only the first primary vertex is considered. In order to study the possible differences in the $p_{T}$ distribution, the full data sample of the analysis is divided into three regions: a) $|z|<100 \mathrm{~mm}$, b) $100 \mathrm{~mm}<|z|<200 \mathrm{~mm}$ and c) $|z|>200 \mathrm{~mm}$. For each run, the corresponding luminosity is determined by re-weighting its luminosity according to the fraction of events occurring in the selected $\mathrm{z}$ region. The resulting luminosity is then applied separately to the $p_{T}$ distribution of each sample. Figure 8.3 shows the normalized $p_{T}$ distribution for the different samples as well as for the total sample. The full dataset from 2010 is used, as for the standard analysis. After the event selection, about 215 million remain, that are distributed into the different $\mathrm{z}$ samples as follows: a) $89.8 \%$, b) $10.1 \%$, and c) $0.06 \%$ [2].

While the distributions of the different samples mostly agree with the total sample for $p_{T}<80 \mathrm{GeV}$ and $p_{T} \geq 260 \mathrm{GeV}$ within their errors, b) deviates by about $5 \%$ for $80 \leq p_{T}<260 \mathrm{GeV}$ and c) shows even larger deviations, but the effect is negligible due to its very limited statistics. Considering that b) only represents $10.1 \%$ of the total events, its deviation does not significantly contribute to the final spectrum. Overall, the conclusion is that the effect of possible mis-modelling of the event vertex 
distribution is small enough to be disregarded.

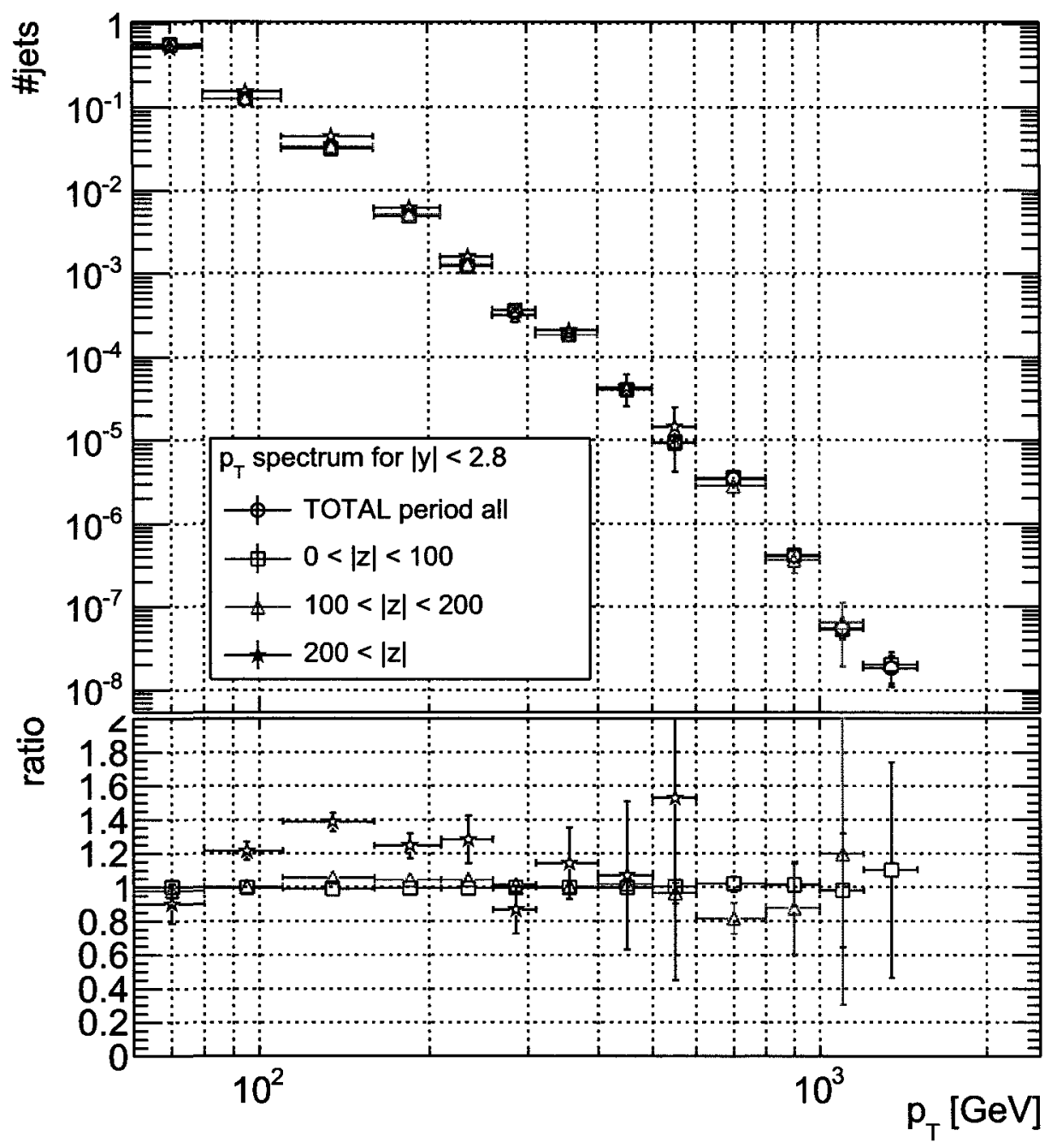

Figure 8.3: Jet $p_{T}$ spectra for various classes of $\mathrm{z}$ distribution of the primary vertex. 


\subsection{Data Correction and Measurement Uncertain- ties}

The Jet Energy Scale (JES) is the dominant uncertainty on the jet cross section measurements. Other non-negligible sources of uncertainty include the jet energy resolution and angular resolution. The latter two also introduce biases in the measurements which must be taken into account. This is discussed below.

\subsubsection{Data Unfolding}

The jet energy and $p_{T}$ resolution will naturally cause jets to migrate from bin to bin. If the cross section were constant as a function of $p_{T}$, the amount of jets migrating out would equal the number migrating in, effectively cancelling the effect. However, the $p_{T}$ spectrum is not constant but drops steeply as a function of $p_{T}$. This creates a situation where more jets migrate into a given $p_{T}$ bin from the low $p_{T}$ than from high $p_{T}$. Consequently the number of jets observed in a given bin will be larger than the true number. This effect can be corrected for by using MC simulations to predict the jet excess. The correction is performed by plotting both the MC truth spectrum and the reconstructed spectrum, and taking the ratio:

$$
C=\frac{\sigma_{M C, t r u t h}}{\sigma_{M C, \text { reco }}} .
$$

The unfolding correction is applied, bin-by-bin, to the measured data spectrum, restoring the particle level cross section. This is referred to as unfolding of data.

Three key factors affect the derivation of the unfolding corrections: the jet energy 
resolution, the angular (rapidity) resolution, and the shape of the spectrum. Systematic uncertainties on these correction factors are derived by varying the jet energy resolution, the jet angular resolution, and the cross section shape in Monte Carlo.

From dijet balance and $\mathrm{E} / \mathrm{p}$ studies of single hadrons, the $p_{T}$ resolution has been verified to within $10 \%$. To assess the impact of a difference between the simulated and real energy resolution, the resolution in the Monte Carlo was artificially worsened. This was done by adding a randomized amount of energy to reconstructed jets in the Monte Carlo simulation, that corresponds to a resolution being worse by $10 \%$. The deviation in the correction factors with $10 \%$ worsened resolution is taken as the systematic uncertainty [2].

To account for uncertainty in the jet angular resolution, the jet rapidity is smeared to correspond to a resolution that is $10 \%$ worse than that predicted by the Monte Carlo. This leads to an uncertainty on the correction factors that is $<1 \%$ for most regions, but up to $5 \%$ for forward regions at high $p_{T}[2]$.

Any change in the jet cross section shape also causes a change in the bin-to-bin migrations. Uncertainties due to the shape of the truth and reconstructed $p_{T}$ spectra in Monte Carlo were assessed by applying a weighting function to both the truth and reconstructed jets that covers the difference between the shape of the spectra in data and Monte Carlo [2].

The default correction factors for the inclusive jet cross section are shown in Figures 8.4, 8.5, 8.6, and 8.7. 


\subsubsection{Estimation of the Uncertainty due to JES}

The uncertainty on the inclusive jet cross section measurement from the JES is evaluated using the nominal Pyhtia Monte Carlo sample. The approach taken to derive the uncertainty is a simple one: the $p_{T}$ spectrum for each rapidity bin is plotted after shifting the $p_{T}$ of each jet up and down by one standard deviation of the JES uncertainty. The shifted spectra are then compared to the nominal spectrum. The relative shift of the jet yield in each bin is taken as the uncertainty on the cross section.

A slight complication arises from the fact that the JES uncertainty is dependent on the amount of pile up. More precisely, the JES uncertainty is proportional to $\left(N_{\mathrm{PV}}-1\right) / p_{T}$, and is quite small for high $p_{T}$ jets and is not present in events with only one primary vertex. To account for the effects of pile up, the following strategy is applied: For each number of primary vertices, $N_{\mathrm{PV}}=1, . ., 9$, a shifted $p_{T}$ spectrum is plotted by shifting the $p_{T}$ of each jet up and down by one sigma of the JES uncertainty. This results in 18 different spectra for each $y$ bin: 9 up-shifted and 9 down-shifted. Each of the 9 pairs of spectra provides an uncertainty on the cross section due to the JES for a specific $N_{\mathrm{PV}}=1, . ., 9$. The final uncertainty on the cross section, $\delta_{\sigma}$, is obtained by weighting each of the 9 different uncertainties and adding them:

$$
\delta_{\sigma}=\sum_{N_{\mathrm{PV}}=1}\left[\delta_{N_{\mathrm{PV}}} \times P\left(N_{\mathrm{PV}}\right)\right]
$$

where $\delta_{N_{\mathrm{PV}}}$ is the uncertainty on the cross section due to JES with $N_{\mathrm{PV}}$ primary vertices, and $P\left(N_{\mathrm{PV}}\right)$ is the probability of having $N_{\mathrm{PV}}$ primary vertices. The probability distributions are obtained from the same dataset that is used for the cross section measurements. Each $p_{T^{-}} y$ bin has its own $N_{\mathrm{PV}}$ probability distribution because each 
bin uses a different subset of data; each bin has one designated trigger which collects the data. For example, the low- $p_{T}$ bins mostly use the very early data where the LHC luminosity was quite low and so the Minimum Bias trigger was not heavily prescaled. For these bins then, there is little pile up and hence most events have $N_{\mathrm{PV}}=1$.

Figure 8.8 shows a few sample $N_{\mathrm{PV}}$ distributions used in the cross section uncertainty derivation. The total uncertainties on the cross section are shown for each jet $y$-bin as a function of jet $p_{T}$ in Figures 8.9 and 8.10. 

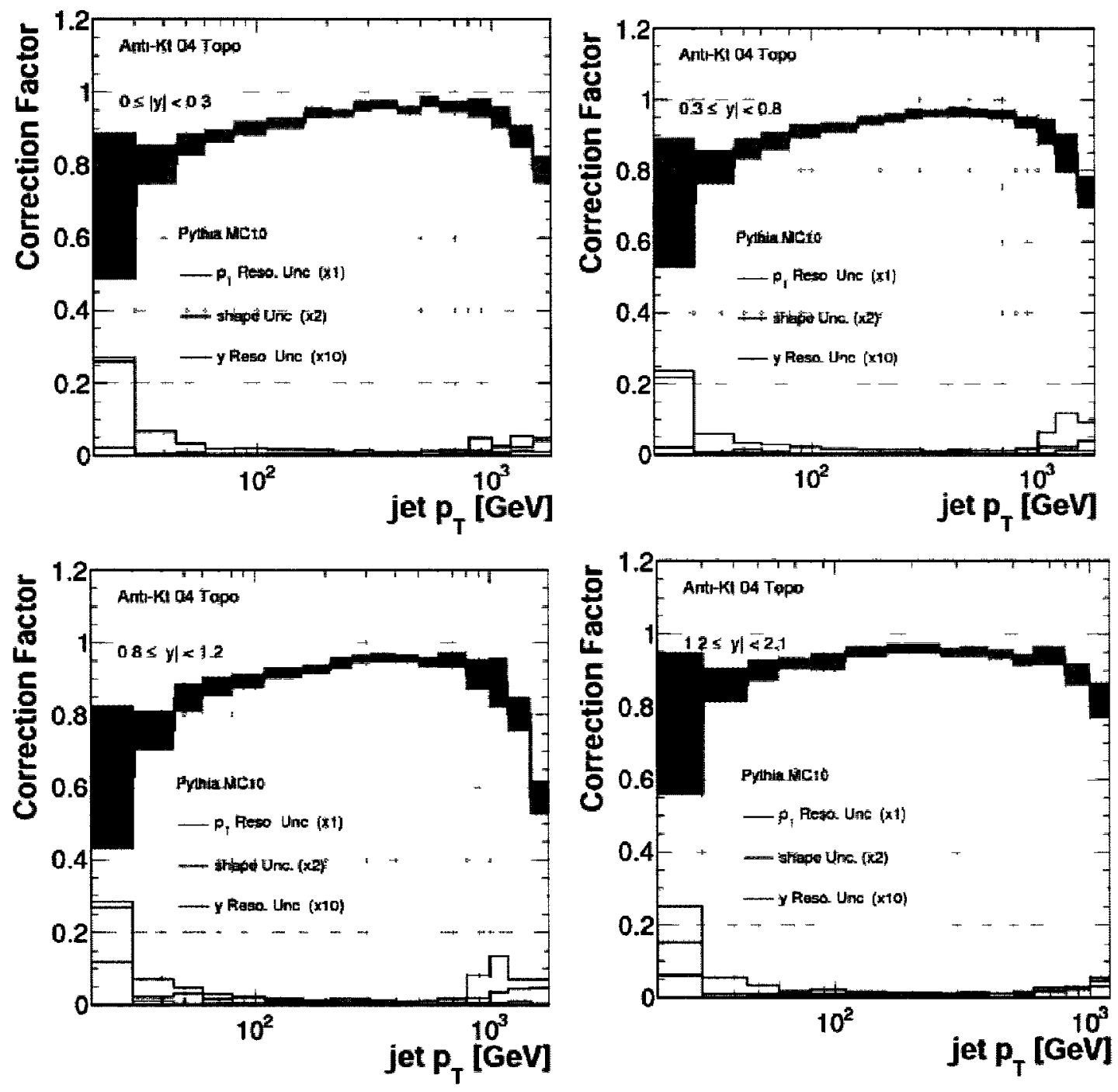

Figure 8.4: Unfolding Correction factors as a function of jet $p_{T}$ for $R=0.4$ jets as defined in Equation (8.9) for the four central rapidity bins along with systematic uncertainties on these factors due to uncertainties in the jet energy and angular resolution, and the MC spectrum shape. 

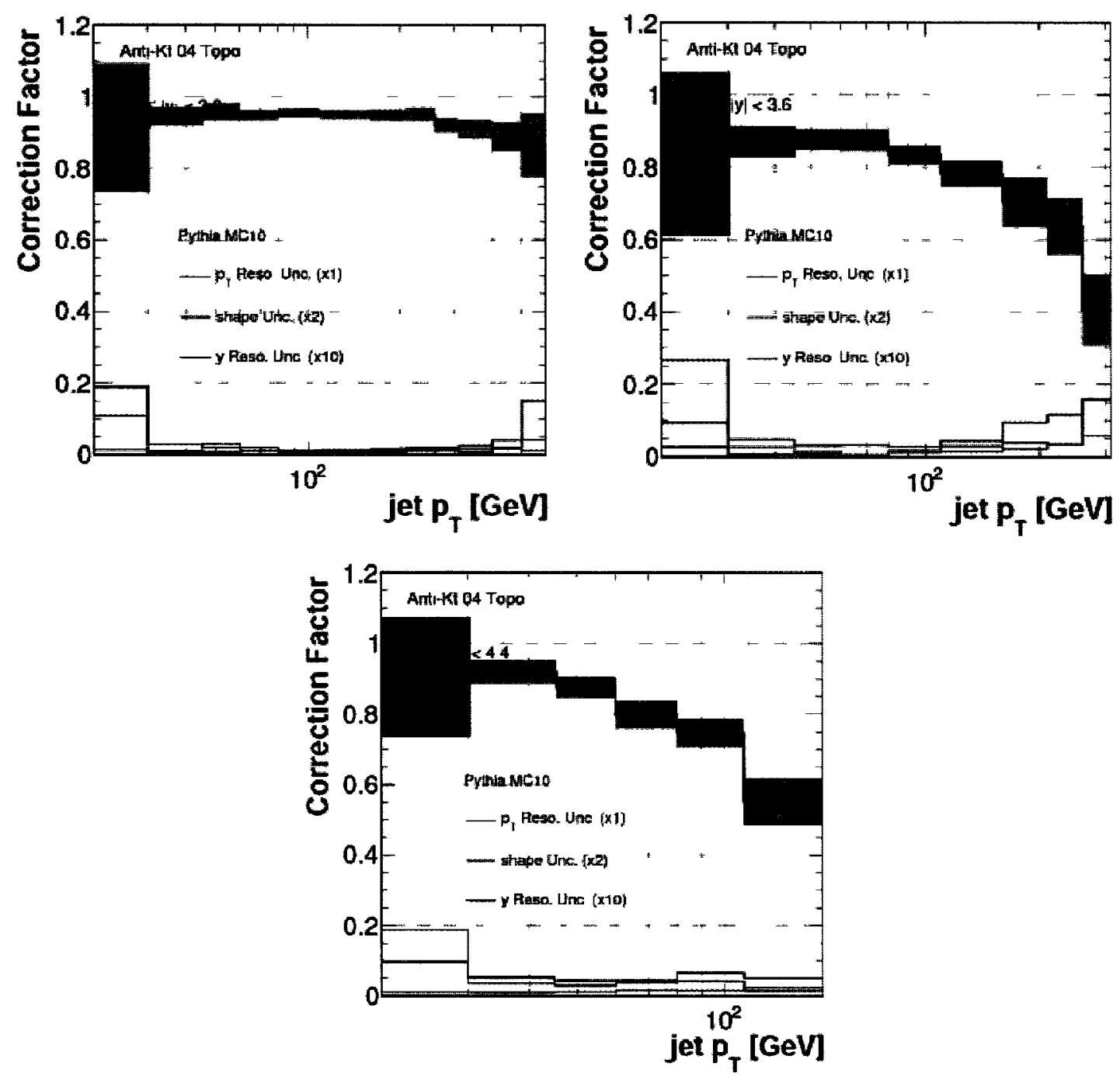

Figure 8.5: Unfolding Correction factors as a function of jet $p_{T}$ for $R=0.4$ jets as defined in Equation (8.9) for the three forward rapidity bins along with systematic uncertainties on these factors due to uncertainties in the jet energy and angular resolution, and the MC spectrum shape. 

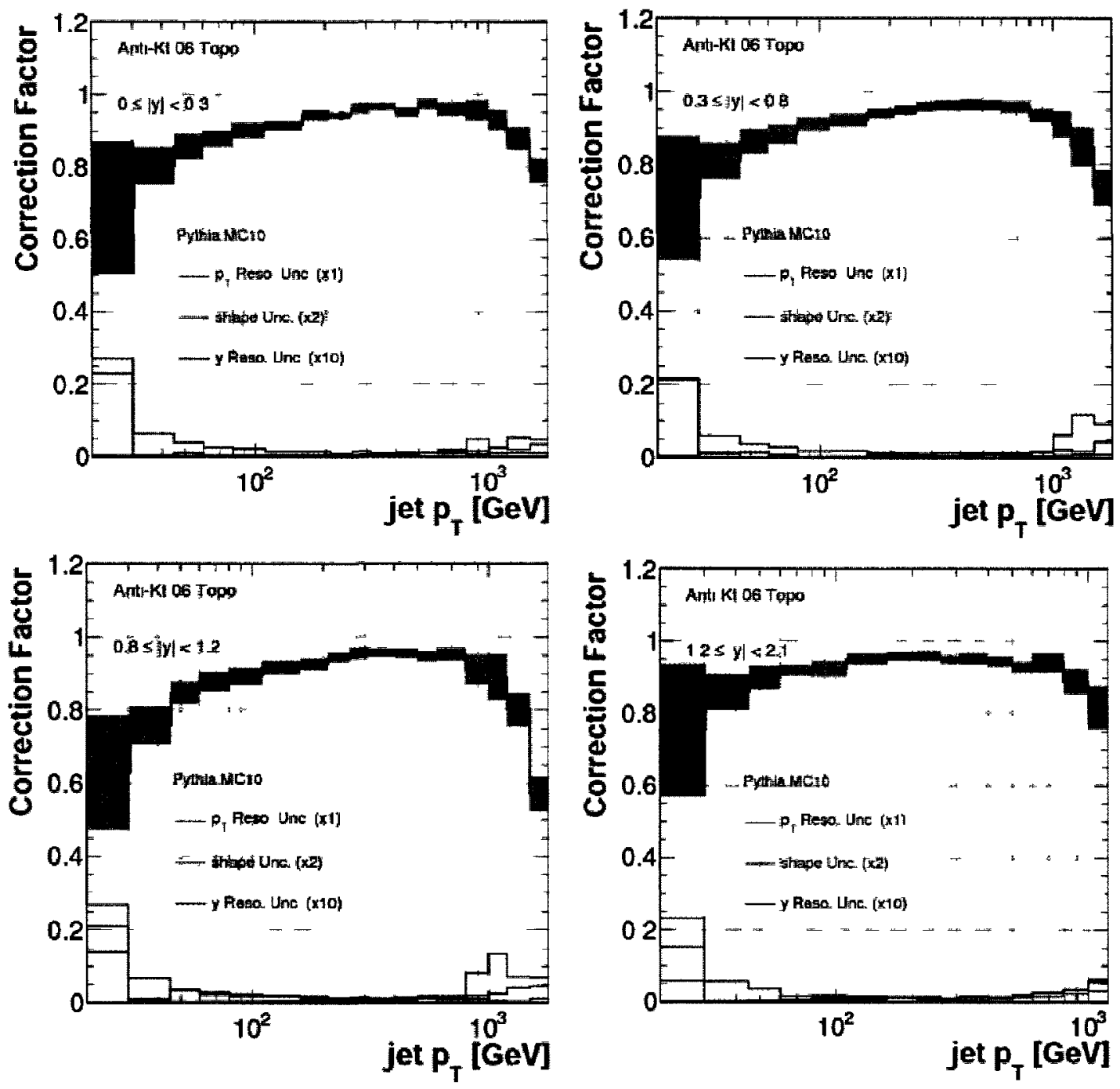

Figure 8.6: Unfolding Correction factors as a function of jet $p_{T}$ for $R=0.6$ jets as defined in Equation (8.9) for the four central rapidity bins along with systematic uncertainties on these factors due to uncertainties in the jet energy and angular resolution, and the MC spectrum shape. 

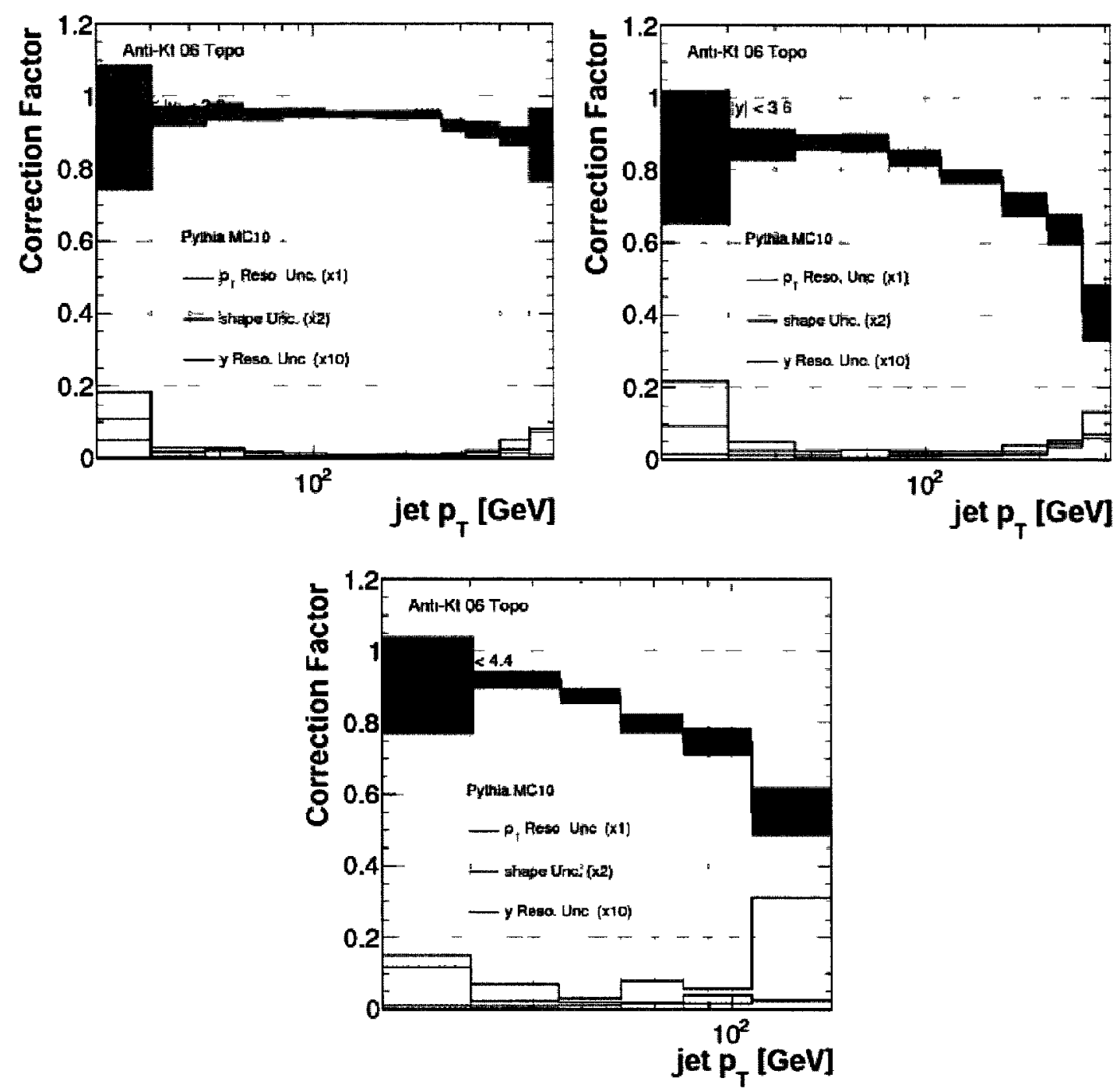

Figure 8.7: Unfolding Correction factors as a function of jet $p_{T}$ for $R=0.6$ jets as defined in Equation (8.9) for the three forward rapidity bins along with systematic uncertainties on these factors due to uncertainties in the jet energy and angular resolution, and the $\mathrm{MC}$ spectrum shape. 


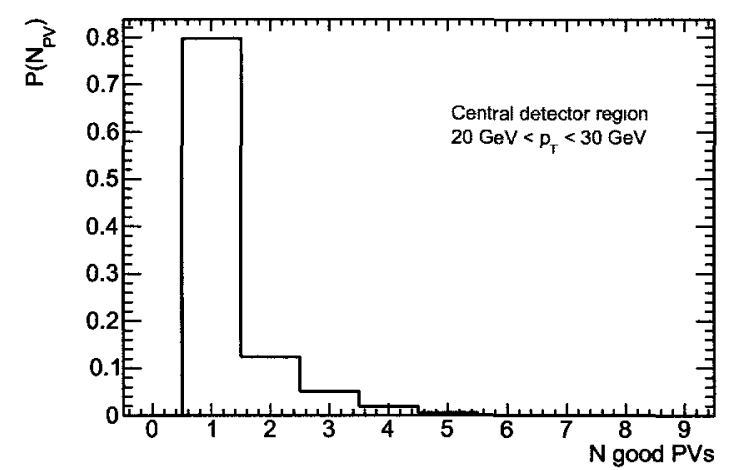

(a)

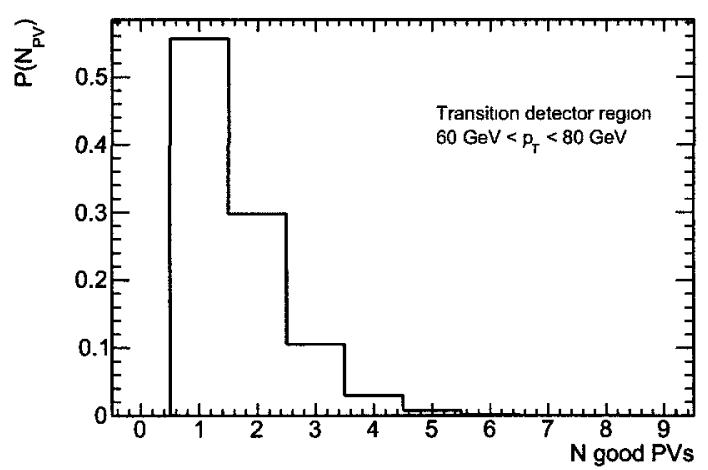

(b)

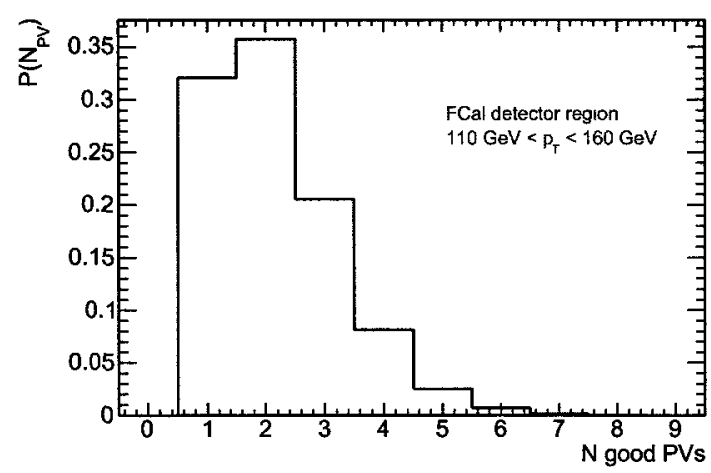

(c)

Figure 8.8: Probability of having $N_{\mathrm{PV}}$ primary vertices in a given collision event and in a particular $y-p_{T}$ bin: (a) the central region with $0.3<|\eta|<0.8$ and $20 \mathrm{GeV}<$ $p_{T}<30 \mathrm{GeV}$, (b) the transition region $\left(2.8<|\eta| \leq 3.6\right.$ and $60 \mathrm{GeV}<p_{T}<80 \mathrm{GeV}$, and (c) the forward region $\left(3.6<|\eta| \leq 4.5\right.$ and $\left.110 \mathrm{GeV}<p_{T}<160 \mathrm{GeV}\right)$. 

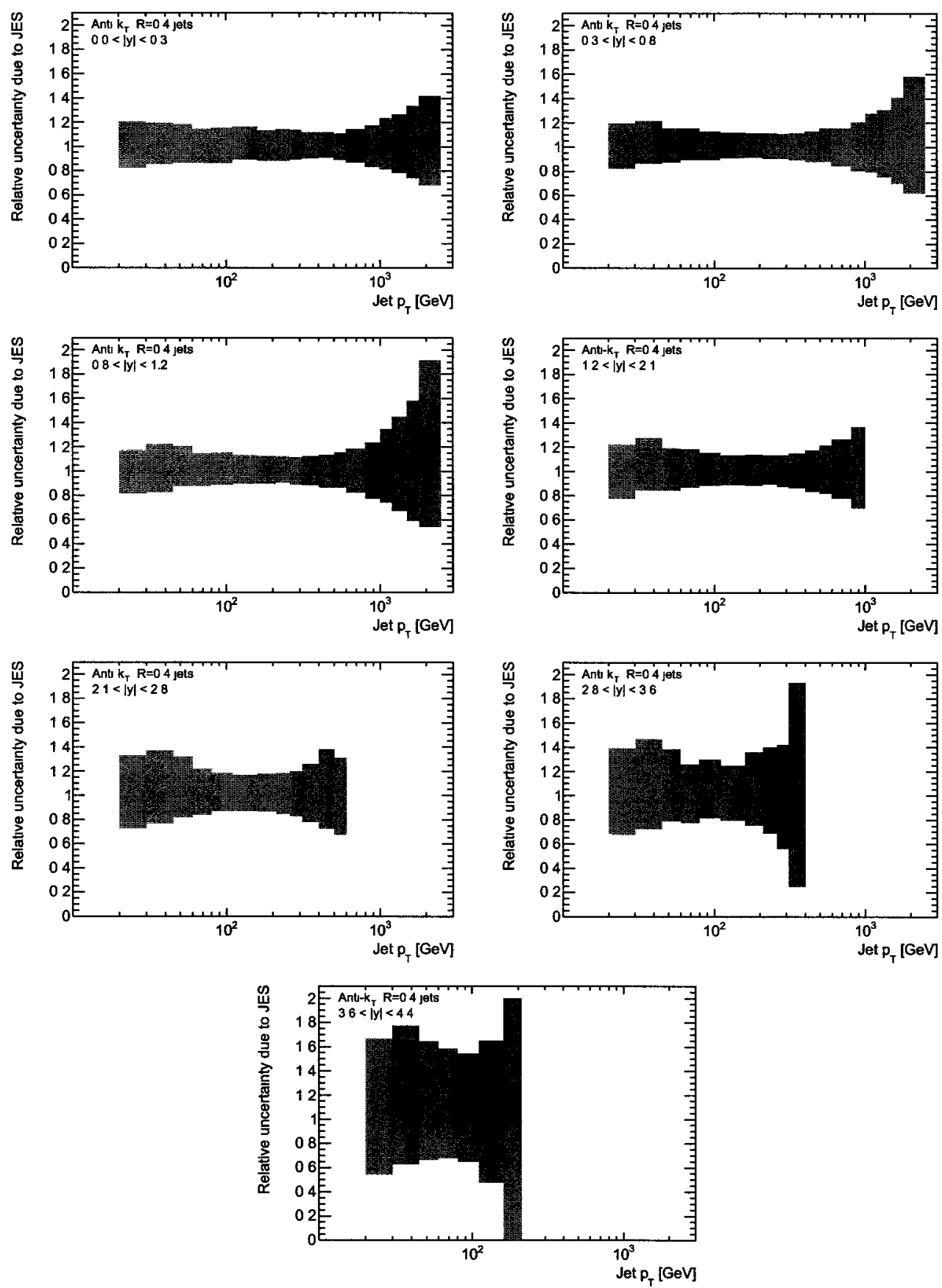

Figure 8.9: Systematic uncertainty of the inclusive jet cross section measurement due to JES for anti- $k_{T}$ jets with $R=0.4$ derived using the shape of the MC10 Pythia dijet sample along with the JES uncertainty. The JES uncertainty due to pile up is taken into account using a conservative estimate of the pile-up uncertainty and the $N_{\mathrm{PV}}$ distribution observed in data. 

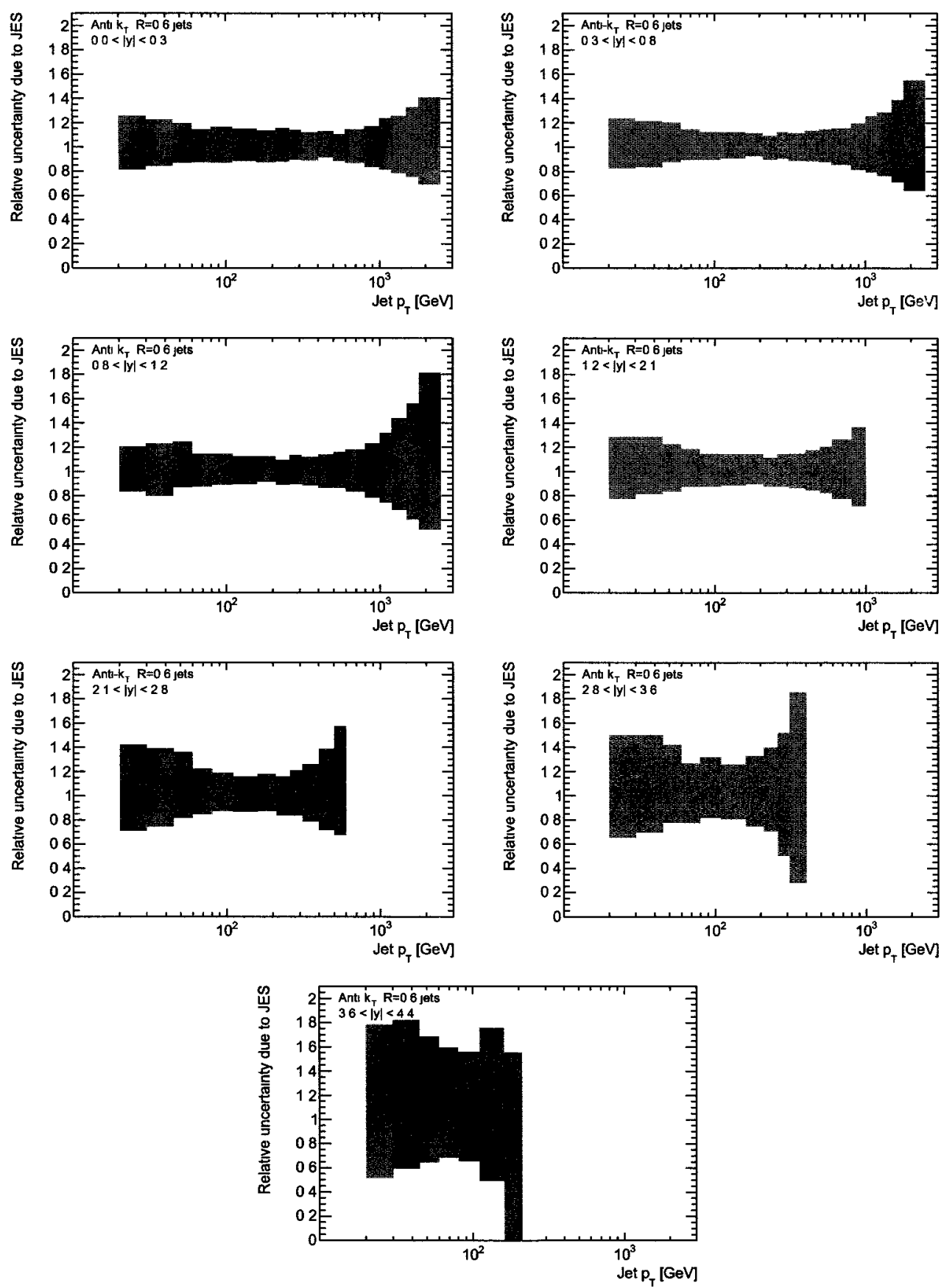

Figure 8.10: Systematic uncertainty of cross section measurement due to JES for anti$k_{T}$ jets with $R=0.6$ derived using the shape of the MC10 Pythia dijet sample along with the JES uncertainty. The JES uncertainty due to pile up is taken into account using a conservative estimate of the pile-up uncertainty and the $N_{\mathrm{PV}}$ distribution observed in data. 


\subsection{Results and Discussion}

The results of the inclusive jet and dijet cross section analysis presented here was a group effort of about 36 individuals, myself included, from the ATLAS Collaboration. All plots of the results presented here are taken from the ATLAS conference note [50], presented at the Moriond QCD conference in March 2011, and its supporting note [2]. A publication of the analysis, with possible upgrades and extensions, is scheduled to take place in the European Physical Journal (EPJ) by the end of the summer of 2011.

\subsubsection{Inclusive Jet Cross Sections}

The inclusive jet cross sections are presented as functions of jet $p_{T}$ in bins of jet rapidity. The double-differential inclusive jet cross section in $7 \mathrm{TeV}$ proton-proton collisions is shown in Figures 8.11 and 8.12 for jets reconstructed with the anti- $k_{T}$ algorithm with $R=0.4$ and $R=0.6$, respectively. The ratio of the measured cross section with respect to the theoretical prediction from NLO pQCD corrected for nonperturbative effects, separated into different rapidity regions, is shown for $R=0.4$ and $R=0.6$ in Figures 8.13-8.14 and 8.15-8.16, respectively.

The measurement extends from jet transverse momentum of $20 \mathrm{GeV}$ to almost $1.5 \mathrm{TeV}$, spanning 3 orders of magnitude in $p_{T}$ and seven orders of magnitude in cross section. Detector-level cross sections have been corrected for all detector effects using the unfolding procedure described in Section 8.5.1. The results are compared to NLO pQCD predictions corrected for non-perturbative effects, where the theoretical uncertainties from scale variations, parton distribution functions, and non-perturbative corrections have been accounted for. The data and theory predictions are in good 
agreement within experimental and theoretical uncertainties. As expected, the largest disagreement occurs in the unexplored regions of phase space, namely in the high $p_{T}$ and very forward rapidity bins where the discrepancy between the data and the theoretical prediction is about $70 \%$.

The data are compared with predictions obtained with different PDF sets. In Figures $8.17-8.20$, the theoretical error bands in the predictions obtained by using CTEQ 6.6, MSTW 2008, NNPDF 2.1, and HERAPDF 1.5 are shown compared to the measured cross sections, where all data and theoretical predictions are normalized to those obtained by using CTEQ 6.6. Agreement of the theory predictions using different PDF set central values is observed for these PDF sets.

The comparison of data with the Powheg prediction, using the MSTW 2008 NLO $\mathrm{PDF}$ set, is shown for anti- $k_{T}$ jets with $R=0.4$ in different rapidity regions in Figures 8.21 and 8.22. The markers show the comparison with the Powheg prediction showered with Pythia and Herwig, as well as the NLO pQCD prediction derived by using the MSTW 2008 NLO PDF set, which is used as common normalization. The same plots are shown for anti- $k_{T}$ jets with $R=0.6$, in Figures 8.23 and 8.24.

Some differences exist between Powheg showered with Pythia and Herwig. The NLO ME is the same for the two Powheg predictions, and it was also found to be in good agreement with the pure parton-level ME calculation from NLOJet++ before soft corrections. Thus the difference between the two predictions of Powheg coupled with the two different shower implementations may be taken as an indication of the uncertainty due to the leading-logarithm approximation used in the parton shower. Scale uncertainties are not shown for Powheg, but may be assumed to be similar to those for NLOJet++. 
Within these uncertainties, the Powheg predictions are consistent both with the data and with NLOJet++. There is a trend for Powheg to lie above the data and NLOJet++ at low $p_{T}$, and to lie below NLOJet++ (but closer to the data) in the high- $p_{T}$ region. These are also the regions where the scale uncertainty in NLOJet++ increases, and at low $p_{T}$ the soft corrections have a significant influence, and their uncertainty can be large. 


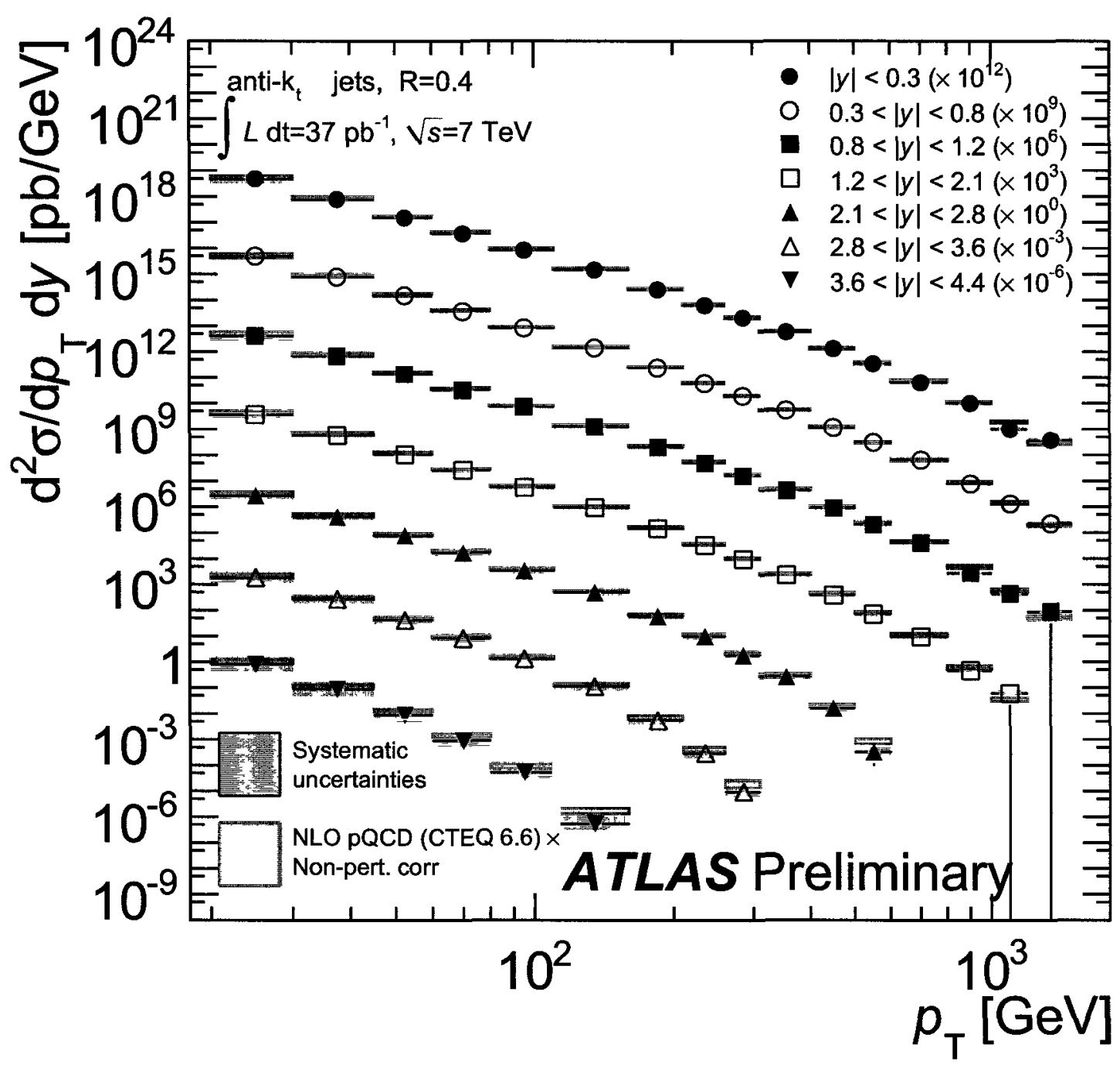

Figure 8.11: Inclusive jet double-differential cross section as a function of jet $p_{T}$ in different regions of $|y|$ for jets identified using the anti- $k_{T}$ algorithm with $R=0.4$. For convenience, the cross sections are multiplied by the factors indicated in the legend. The data are compared to NLO pQCD calculations to which non-perturbative corrections have been applied. The error bars indicate the statistical uncertainty on the measurement, and the shaded band indicates the quadratic sum of the systematic uncertainties, dominated by the jet energy scale uncertainty. There is an additional overall uncertainty of $3 \%$ due to the luminosity measurement that is not shown. The theoretical uncertainty shown is the quadratic sum of uncertainties from the choice of renormalisation and factorisation scales, parton distribution functions, $\alpha_{s}\left(M_{Z}\right)$, and the modelling of non-perturbative effects, as described in the text. 


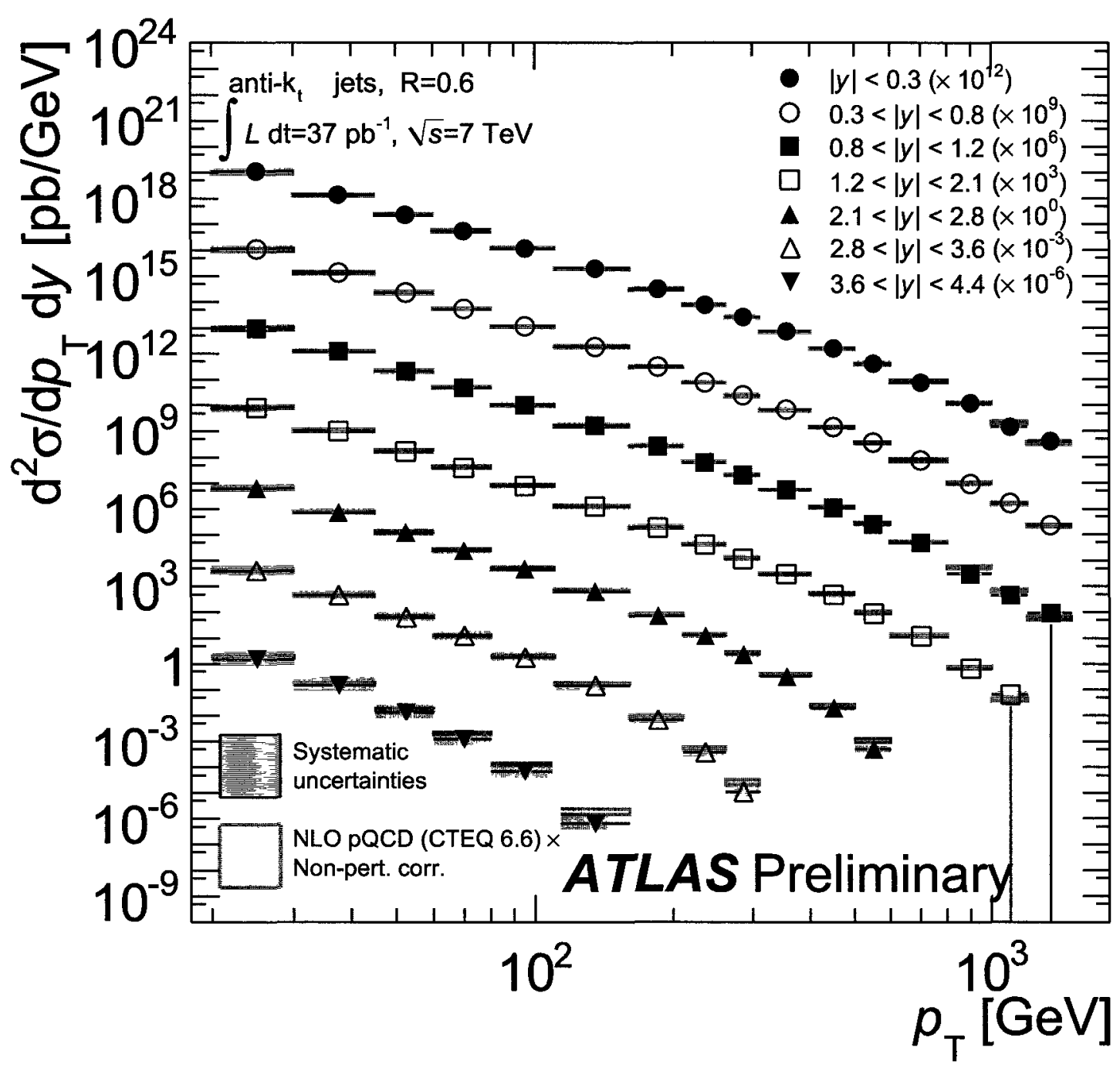

Figure 8.12: Inclusive jet double-differential cross section as a function of jet $p_{T}$ in different regions of $|y|$ for jets identified using the anti- $k_{T}$ algorithm with $R=0.6$. For convenience, the cross sections are multiplied by the factors indicated in the legend. The data are compared to NLO pQCD calculations to which non-perturbative corrections have been applied.The error bars indicate the statistical uncertainty on the measurement, and the shaded band indicates the quadratic sum of the systematic uncertainties, dominated by the jet energy scale uncertainty. There is an additional overall uncertainty of $3 \%$ due to the luminosity measurement that is not shown. The theoretical uncertainty is the quadratic sum of uncertainties from the choice of renormalisation and factorisation scales, parton distribution functions, $\alpha_{s}\left(M_{Z}\right)$, and the mode-ling of non-perturbative effects, as described in the text. 


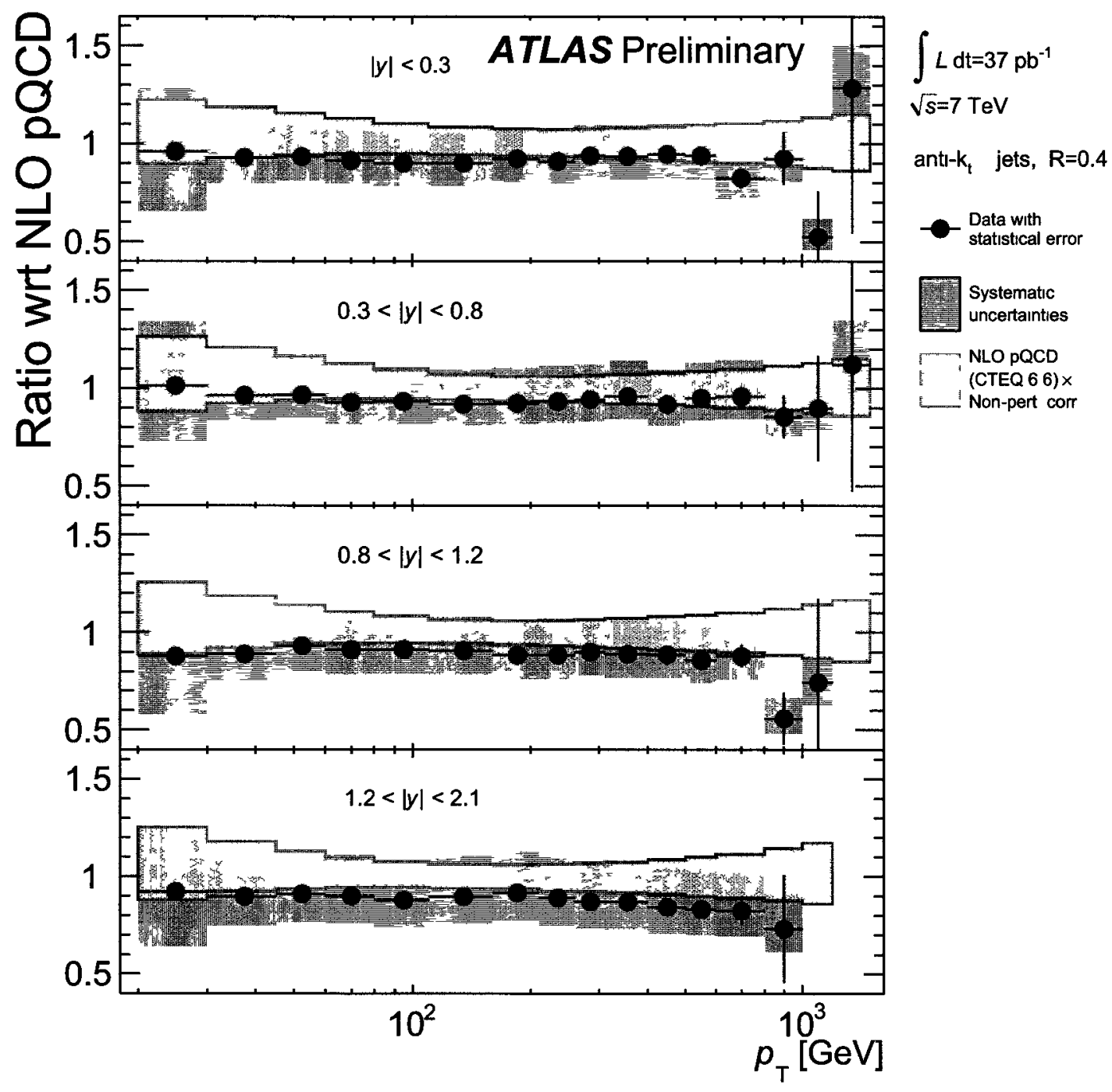

Figure 8.13: Inclusive jet double-differential cross section as a function of jet $p_{T}$ in different regions of $|y|$ for jets identified using the anti- $k_{T}$ algorithm with $R=0.4$. The ratio of the data to the theoretical prediction is shown, and the total systematic uncertainties on the theory and measurement are indicated. The theoretical and experimental uncertainties are calculated as described in Figure 8.11. 


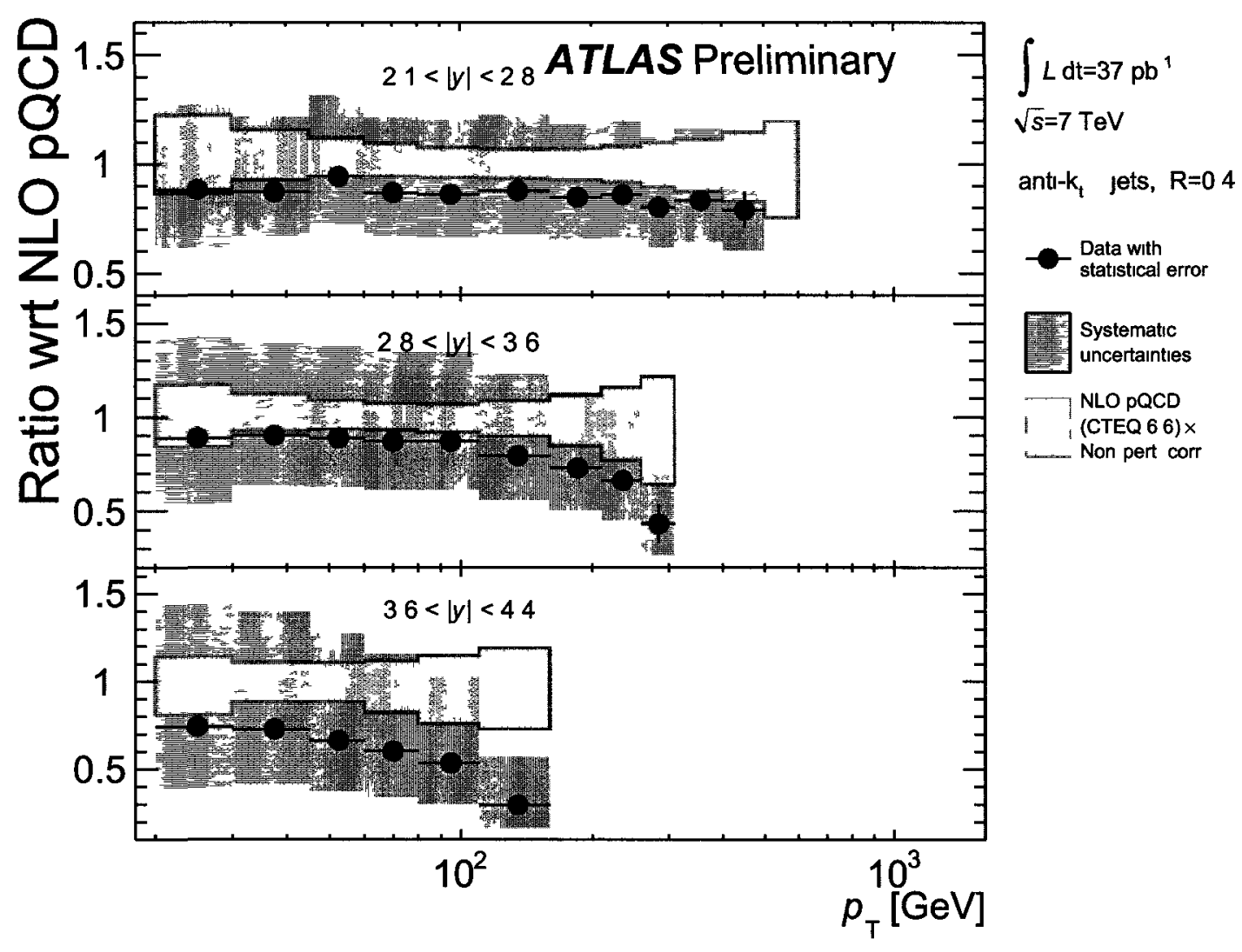

Figure 8 14. Inclusive jet double-differential cross section as a function of jet $p_{T}$ in different forward regions of $|y|$ for jets identıfied using the antı- $k_{T}$ algorithm with $R=0.4$. The ratio of the data to the theoretical prediction is shown, and the total systematic uncertainties on the theory and measurement are indicated. The theoretical and experimental uncertainties are calculated as described in Figure 8.11. 


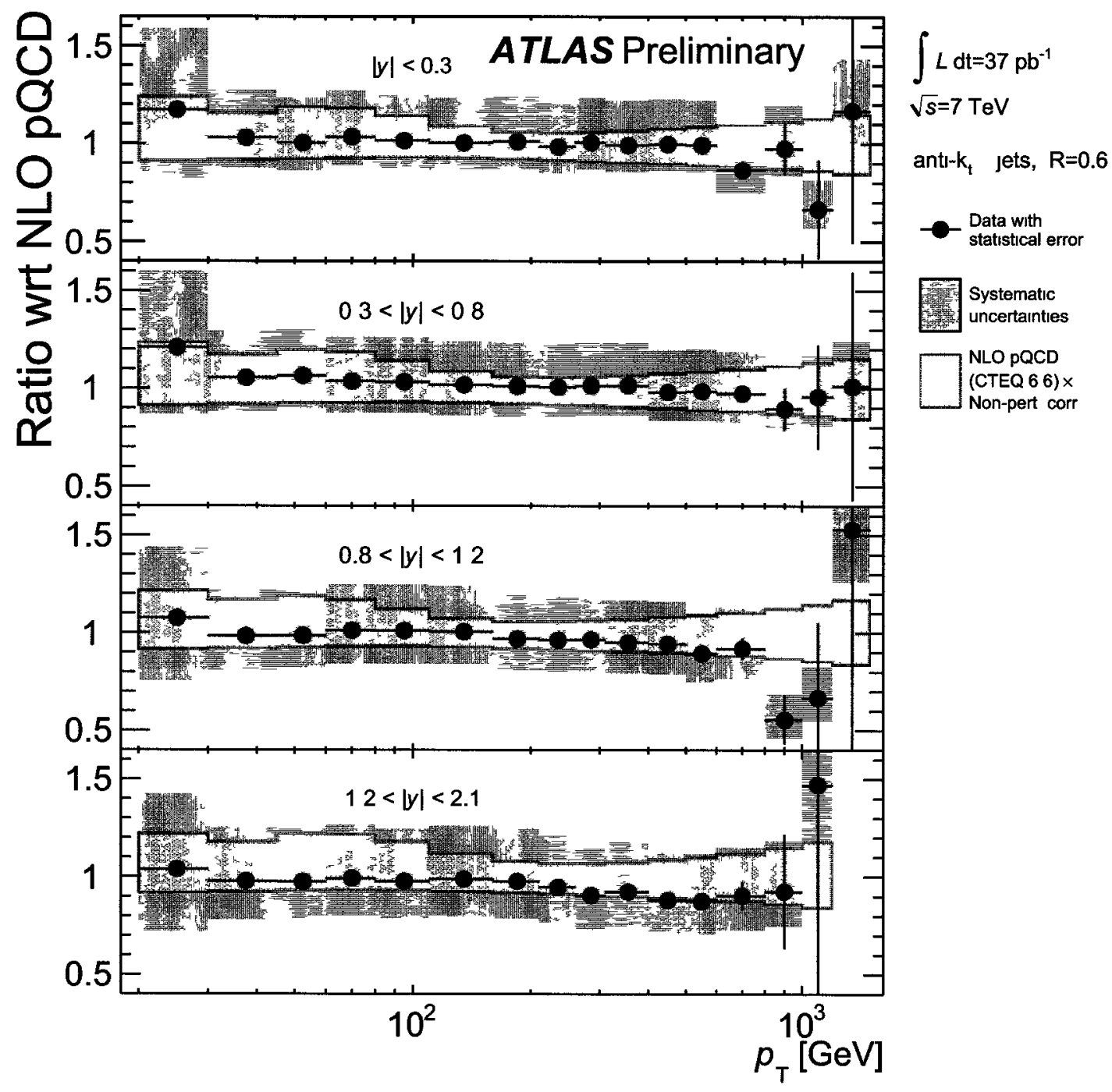

Figure 8.15: Inclusive jet double-differential cross section as a function of jet $p_{T}$ in different regions of $|y|$ for jets identified using the ant1- $k_{T}$ algorithm with $R=0.6$. The ratio of the data to the theoretical prediction is shown, and the total systematic uncertainties on the theory and measurement are indicated. The theoretical and experimental uncertainties are calculated as described in Figure 8.12. 


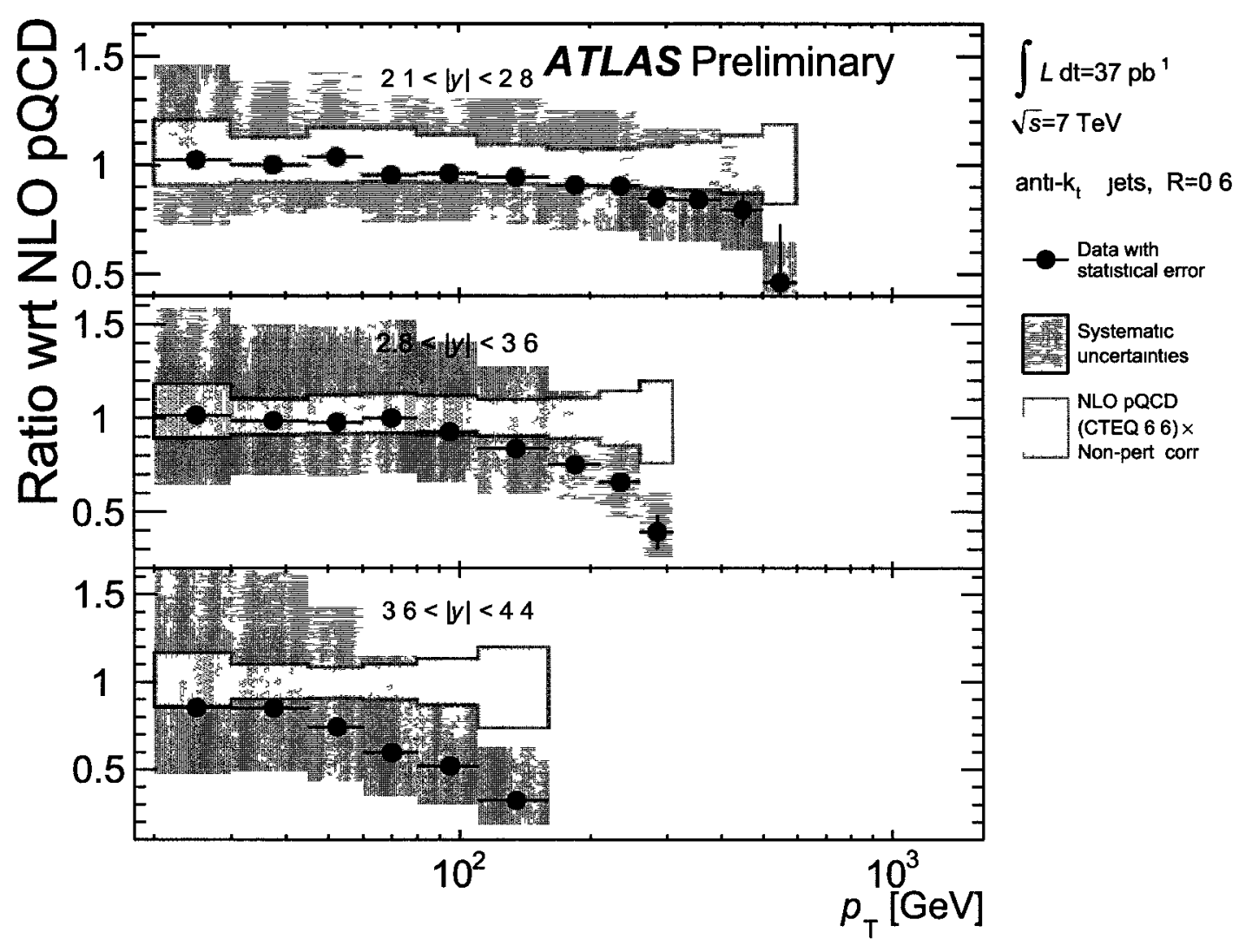

Figure 8.16: Inclusive jet double-differential cross section as a function of jet $p_{T}$ in different forward regions of $|y|$ for jets identified using the anti- $k_{T}$ algorithm with $R=0.6$. The ratio of the data to the theoretical prediction is shown, and the total systematic uncertainties on the theory and measurement are indicated. The theoretical and experimental uncertainties are calculated as described in Figure 8.12. 


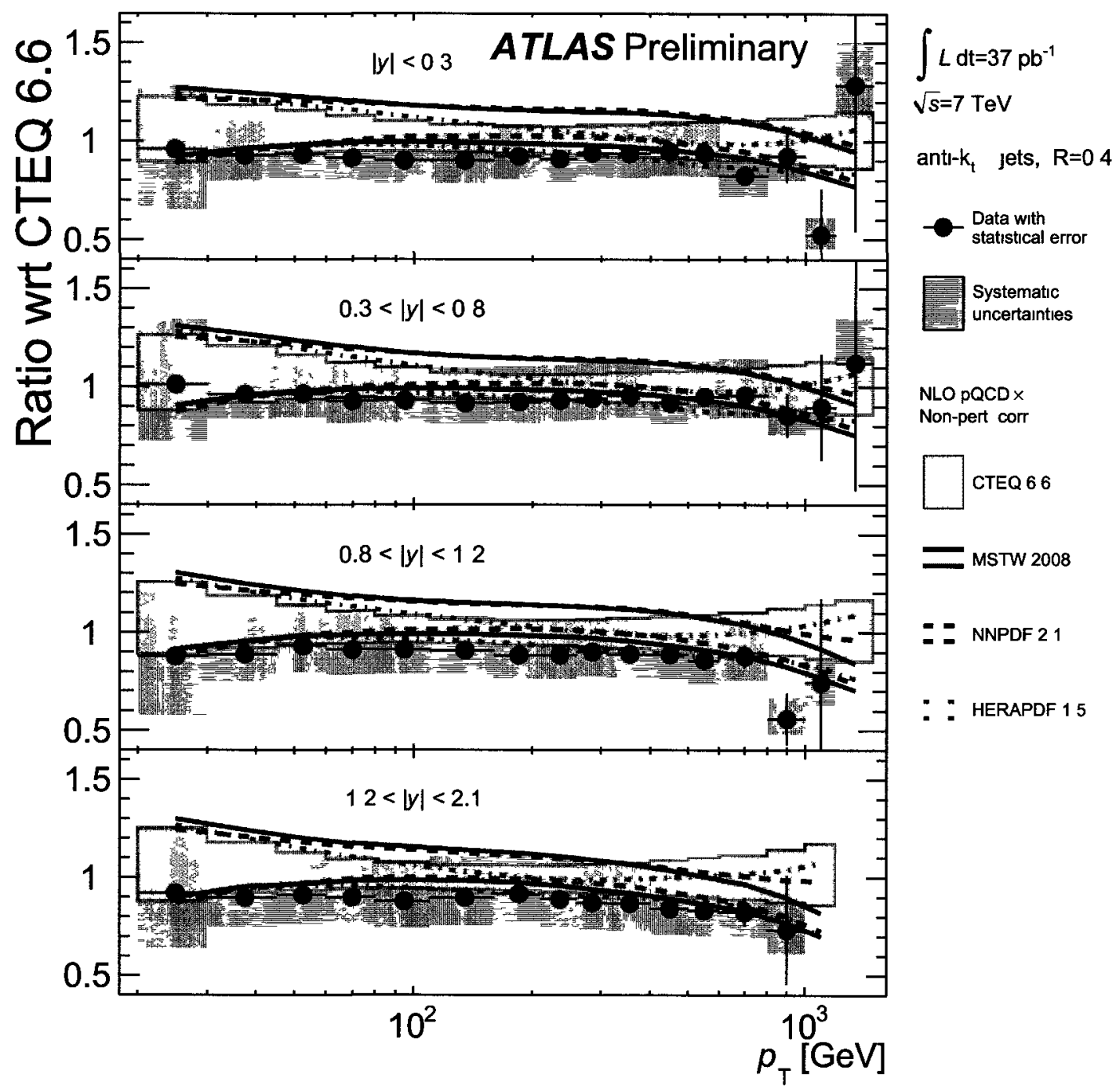

Figure 8.17: Inclusive jet double-differential cross section as a function of jet $p_{T}$ in different central regions of $|y|$ for jets identified using the anti- $k_{T}$ algorithm with $R=0.4$. The theoretical error bands obtained by using different PDF sets (CTEQ 6.6, MSTW 2008, NNPDF 2.1, HERA 1.5) are shown. The data points and the error bands are normalized to the theoretical estimates obtained by using the CTEQ 6.6 PDF set. 


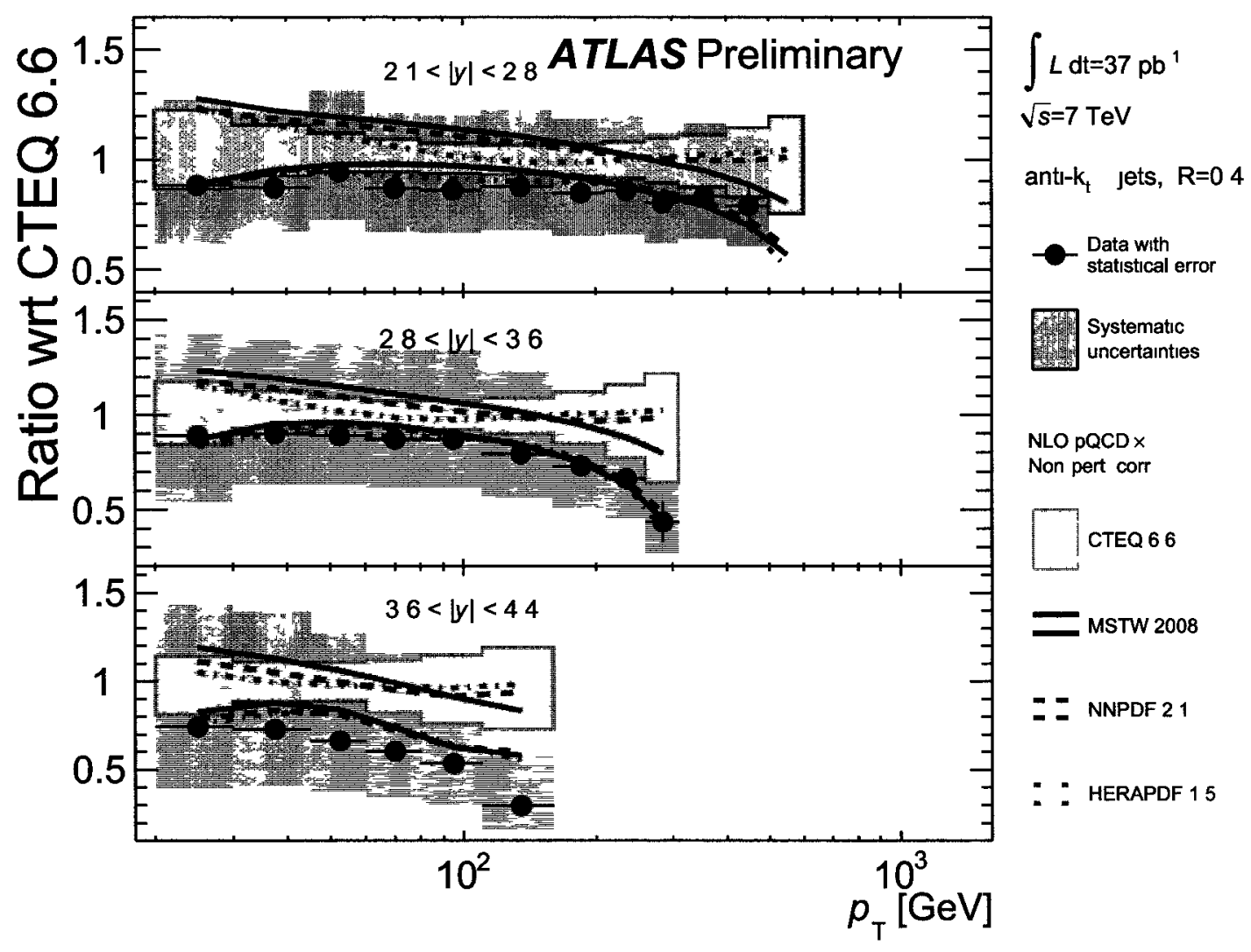

Figure 8.18 Inclusive jet double-differential cross section as a function of jet $p_{T}$ in different forward regions of $|y|$ for jets identified using the ant1- $k_{T}$ algorithm with $R=0.4$. The theoretical error bands obtamed by using different PDF sets (CTEQ 6.6, MSTW 2008, NNPDF 2.1, HERA 1.5) are shown. The data points and the error bands are normalized to the theoretical estimates obtained by using the CTEQ 6.6 PDF set. 


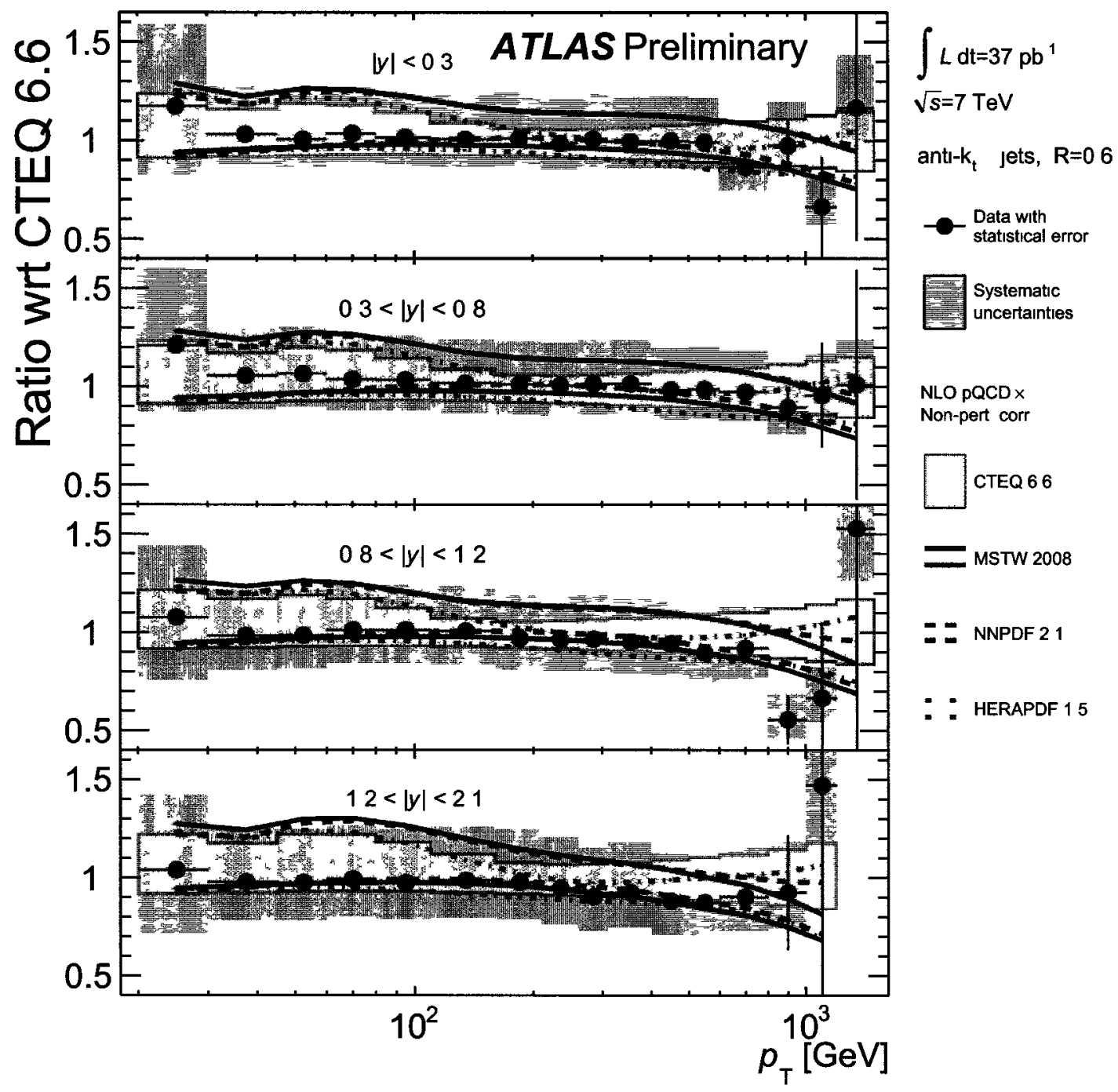

Figure 8.19. Inclusive jet double-differential cross section as a function of jet $p_{T}$ in different central regions of $|y|$ for jets identified using the anti- $k_{T}$ algorithm with $R=0.6$. The theoretical error bands obtained by using different PDF sets (CTEQ 6.6, MSTW 2008, NNPDF 2.1, HERA 1.5) are shown. The data points and the error bands are normalized to the theoretical estimates obtained by using the CTEQ 6.6 PDF set. 


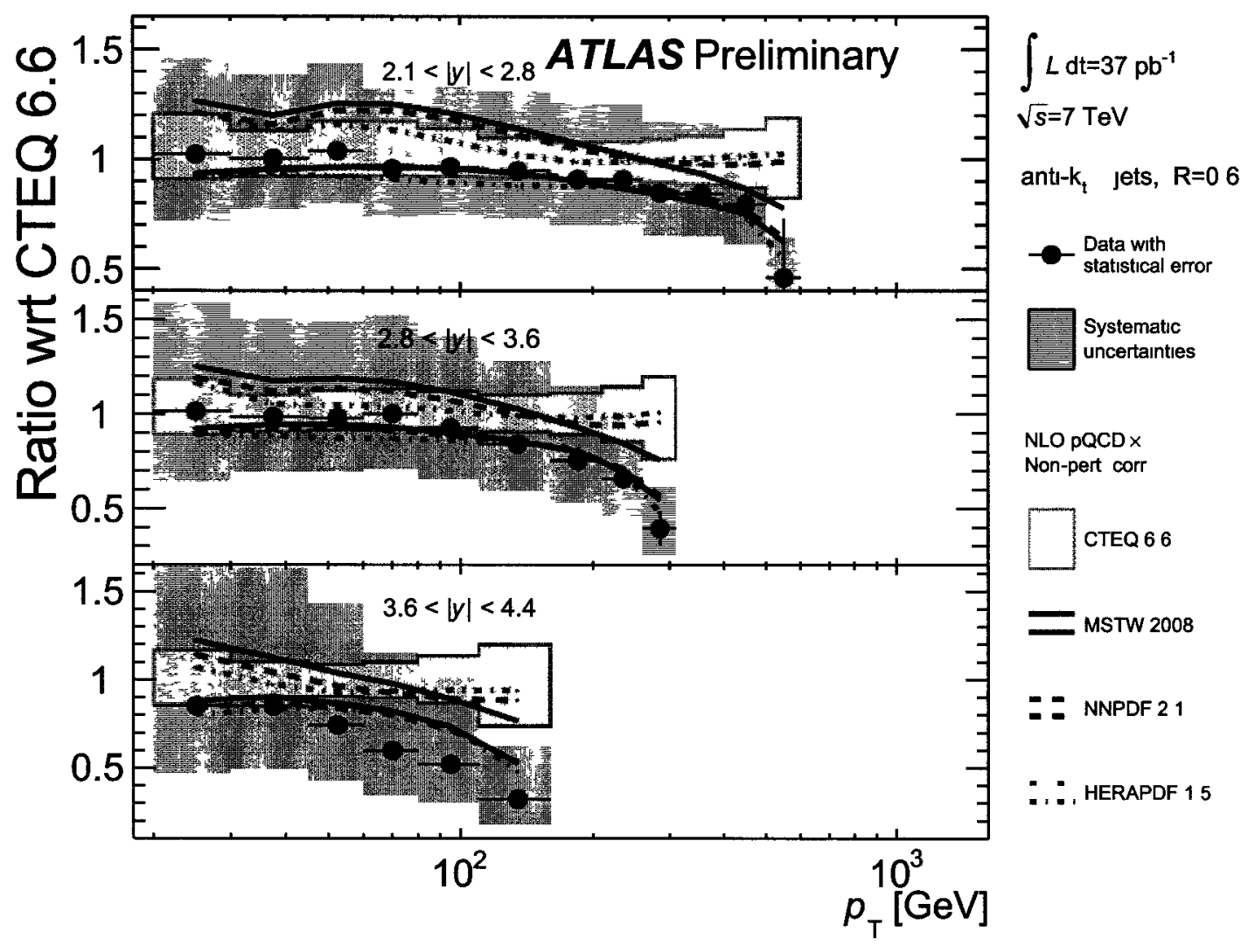

Figure 8.20: Inclusive jet double-differential cross section as a function of jet $p_{T}$ in different forward regions of $|y|$ for jets identified using the anti- $k_{T}$ algorithm with $R=0.6$. The theoretical error bands obtained by using different PDF sets (CTEQ 6.6, MSTW 2008, NNPDF 2.1, HERA 1.5) are shown. The data points and the error bands are normalized to the theoretical estimates obtained by using the CTEQ 6.6 PDF set. 


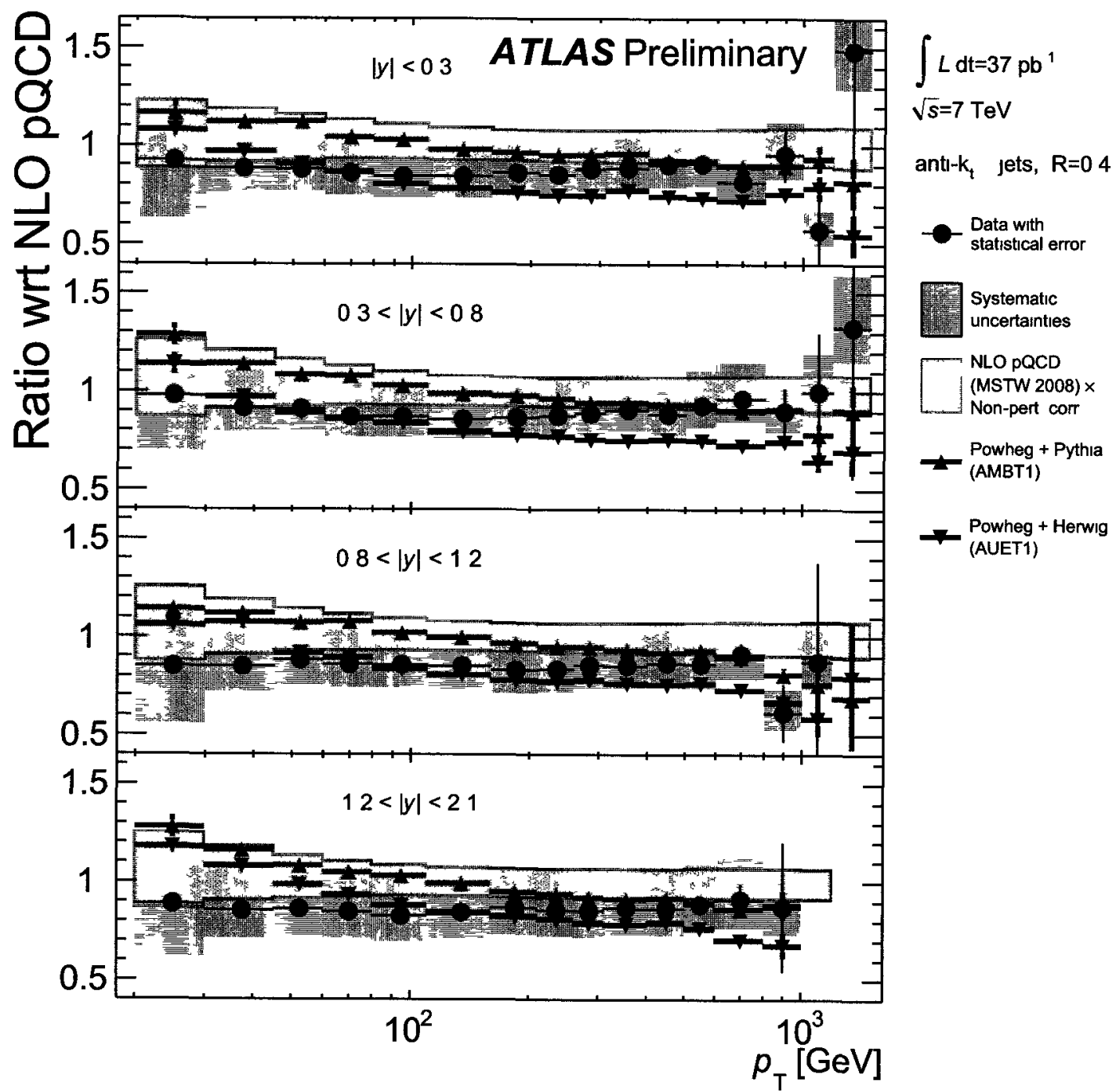

Figure 8.21: Inclusive jet double-differential cross section as a function of jet $p_{T}$ in different regions of $|y|$ for jets identified using the ant1- $k_{T}$ algorithm with $R=0.4$. The ratio of the Powheg predictions showered by Pythia and Herwig to the NLO predictions corrected for the non-perturbative effects is shown. The ratio shows only the statistical uncertainty on the Powheg prediction, and can be compared to the ratio of the data to the NLO pQCD prediction (corrected for non-perturbative effects). The total systematic uncertainties on the theory and measurement are indicated. The NLO pQCD prediction and the Powheg ME calculations use the MSTW PDF set. 


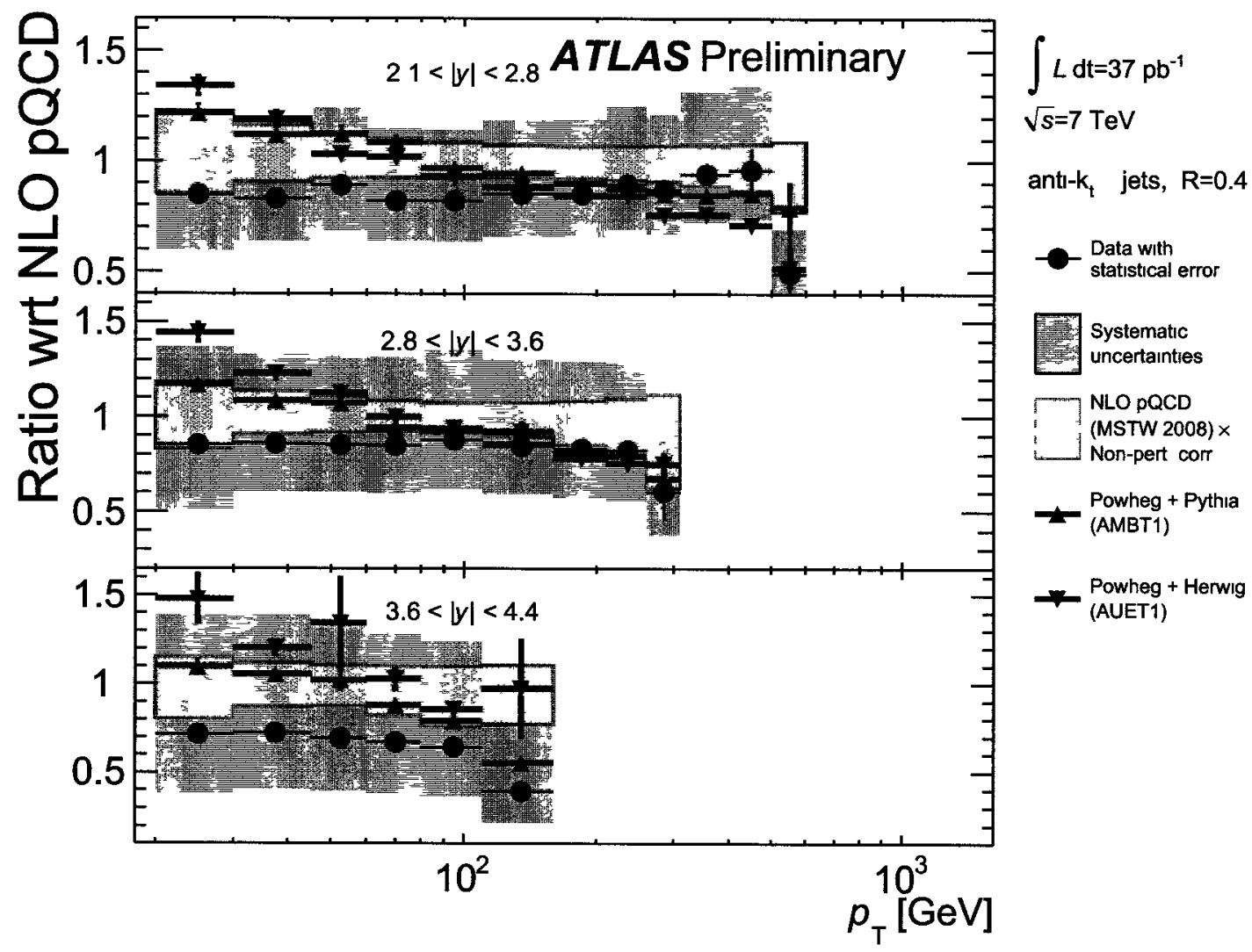

Figure 8.22: Inclusive jet double-differential cross section as a function of jet $p_{T}$ in different forward regions of $|y|$ for jets identified using the anti- $k_{T}$ algorithm with $R=0.4$. The ratio of the Powheg predictions showered by Pythia and Herwig to the NLO predictions corrected for the non-perturbative effects is shown. The ratio shows only the statistical uncertainty on the Powheg prediction, and can be compared to the ratio of the data to the NLO pQCD prediction (corrected for non-perturbative effects). The total systematic uncertainties on the theory and measurement are indicated. The NLO pQCD prediction and the Powheg ME calculations use the MSTW PDF set. 


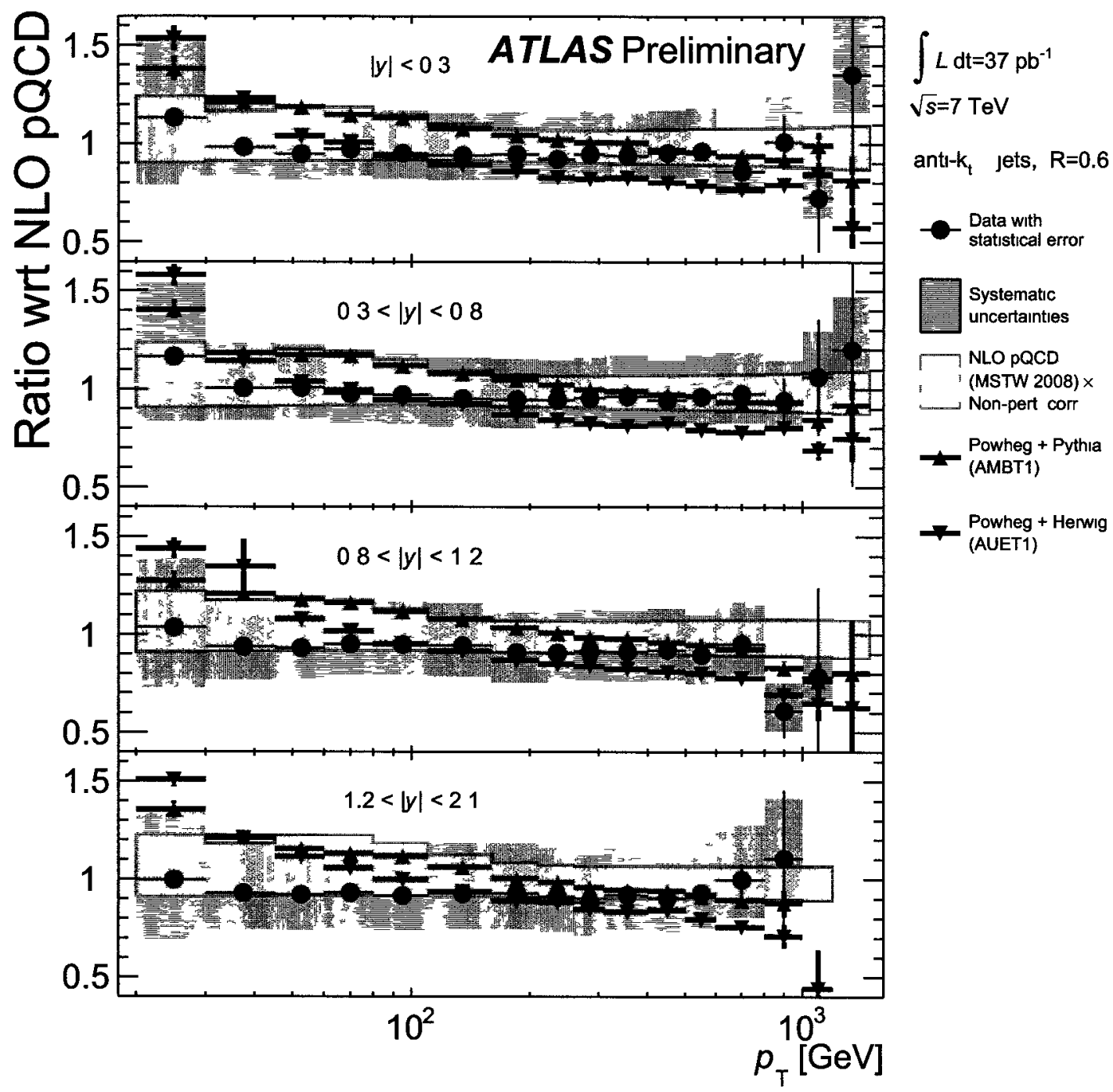

Figure 8.23. Inclusive jet double-differential cross section as a function of jet $p_{T}$ in different regions of $|y|$ for jets identified using the anti- $k_{T}$ algorithm with $R=0.6$. The ratio of the Powheg predictions showered by Pythia and Herwig to the NLO predictions corrected for the non-perturbative effects is shown. The ratio shows only the statistical uncertainty on the Powheg prediction, and can be compared to the ratio of the data to the NLO pQCD prediction (corrected for non-perturbative effects). The total systematic uncertainties on the theory and measurement are indicated. The NLO pQCD prediction and the Powheg ME calculations use the MSTW PDF set. 


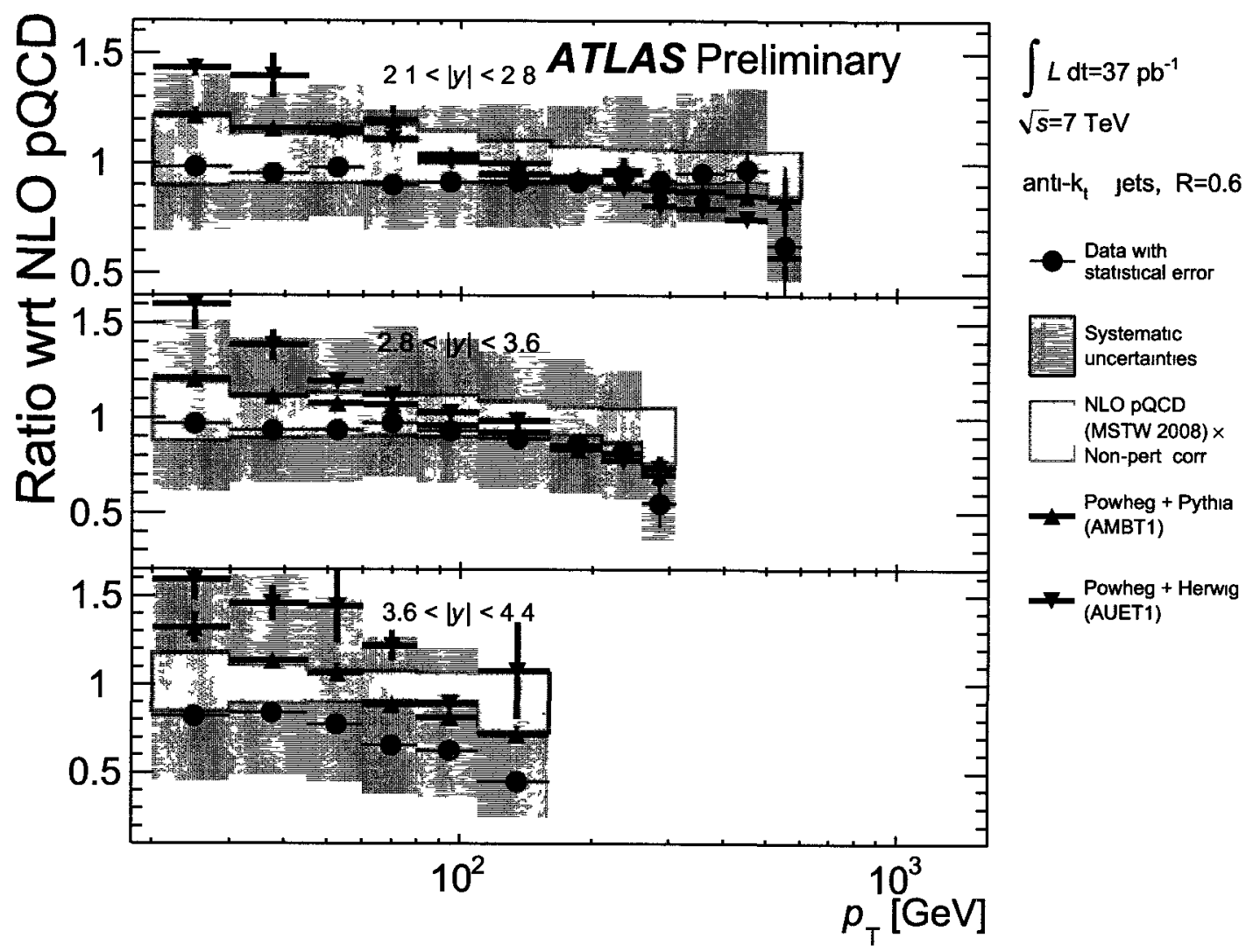

Figure 8.24: Inclusive jet double-differential cross section as a function of jet $p_{T}$ in different forward regions of $|y|$ for jets identified using the anti- $k_{T}$ algorithm with $R=0.6$. The ratio of the Powheg predictions showered by Pythia and Herwig to the NLO predictions corrected for the non-perturbative effects is shown. The ratio shows only the statistical uncertainty on the Powheg prediction, and can be compared to the ratio of the data to the NLO pQCD prediction (corrected for non-perturbative effects). The total systematic uncertainties on the theory and measurement are indicated. The NLO pQCD prediction and the Powheg ME calculations use the MSTW PDF set. 


\subsubsection{Dijet Cross Sections}

The dijet double-differential cross section is measured as a function of the dijet invariant mass for various bins of the rapidity of the most forward (or backward) jet [50].

Figures 8.25 and 8.26 show the double-differential dijet cross section as a function of the dijet mass, for different bins in $|y|_{\max }$ ranging from 0 to 2.8 , for anti- $k_{T}$ jets with $R=0.4$ and $R=0.6$, respectively. The cross section falls rapidly with mass, and extends up to dijet masses above $4 \mathrm{TeV}$. The ratios of the invariant mass with respect to the theory predictions using the CTEQ 6.6 PDF set are shown in Figure 8.27 and 8.28 , respectively. The agreement between data and theory is quite good in the low mass regions but gets much worse in the high mass domain where it is as large as $80 \%$ in certain bins. However, there are not enough statistics to make any firm conclusions. Within statistical uncertainties the data results are consistent with the theoretical predictions.

The dijet data are also compared with predictions obtained using the following NLO PDF sets: CTEQ 6.6, CT10, GJR08 VF, NNPDF 2.1, and MSTW 2008. In particular, the dijet mass spectra for anti- $k_{T}$ jets with $R=0.4$ and $R=0.6$ are shown respectively in Figures 8.29 and 8.30, where the data and the central value predictions from all PDF sets are shown as ratios normalized to the CTEQ 6.6 prediction.

Just accounting for central values and not for their uncertainties, the data seem to disfavour the GJR VF PDF set, undershooting it significantly. The data also mildly undershoot the NNPDF 2.1 and MSTW 2008 NLO PDF sets, though still in agreement within experimental and theoretical uncertainties (omitted for brevity). The best agreement with data is obtained using HERAPDF 1.5, followed by the CT10 
and CTEQ 6.6 PDF sets. ${ }^{1}$

Figures 8.31 and 8.32 show the double-differential dijet cross section as a function of the dijet mass, for different bins in $|y|_{\max }$ ranging from 0 to 2.8 , for anti- $k_{T}$ jets with $R=0.4$. They are compared to Monte Carlo predictions from Powheg, which has a NLO matrix element that is interfaced to a matched parton shower, hadronization, and underlying event modelled by Pythia and Herwig, respectively. The cross section falls rapidly with mass, and extends up to dijet masses above $4 \mathrm{TeV}$. Similar comparisons for anti- $k_{T}$ jets with $R=0.6$ are shown in Figures. 8.33 and 8.34, again for Powheg showered with Pythia and Herwig respectively. A reasonable agreement of Powheg with the data is observed, where the data appear to be bracketed by the predictions using Pythia and Herwig for the showering. In particular the Powheg prediction using Pythia appears to be harder than the data, while the Powheg prediction using Herwig appears to be softer than the data. The difference in predictions using these two shower models, which also have different models of hadronization and underlying event, provides an estimate of the systematic uncertainty associated with the generator. The Powheg predictions agree with the data within this uncertainty.

\footnotetext{
${ }^{1}$ Comparisons to HERAPDF 1.0 , CTEQ 6.5 and NNPDF 2.0 were also performed, but they are not shown as they are very similar to those for HERAPDF 1.5, CTEQ 6.6 and NNPDF 2.1, respectively.
} 


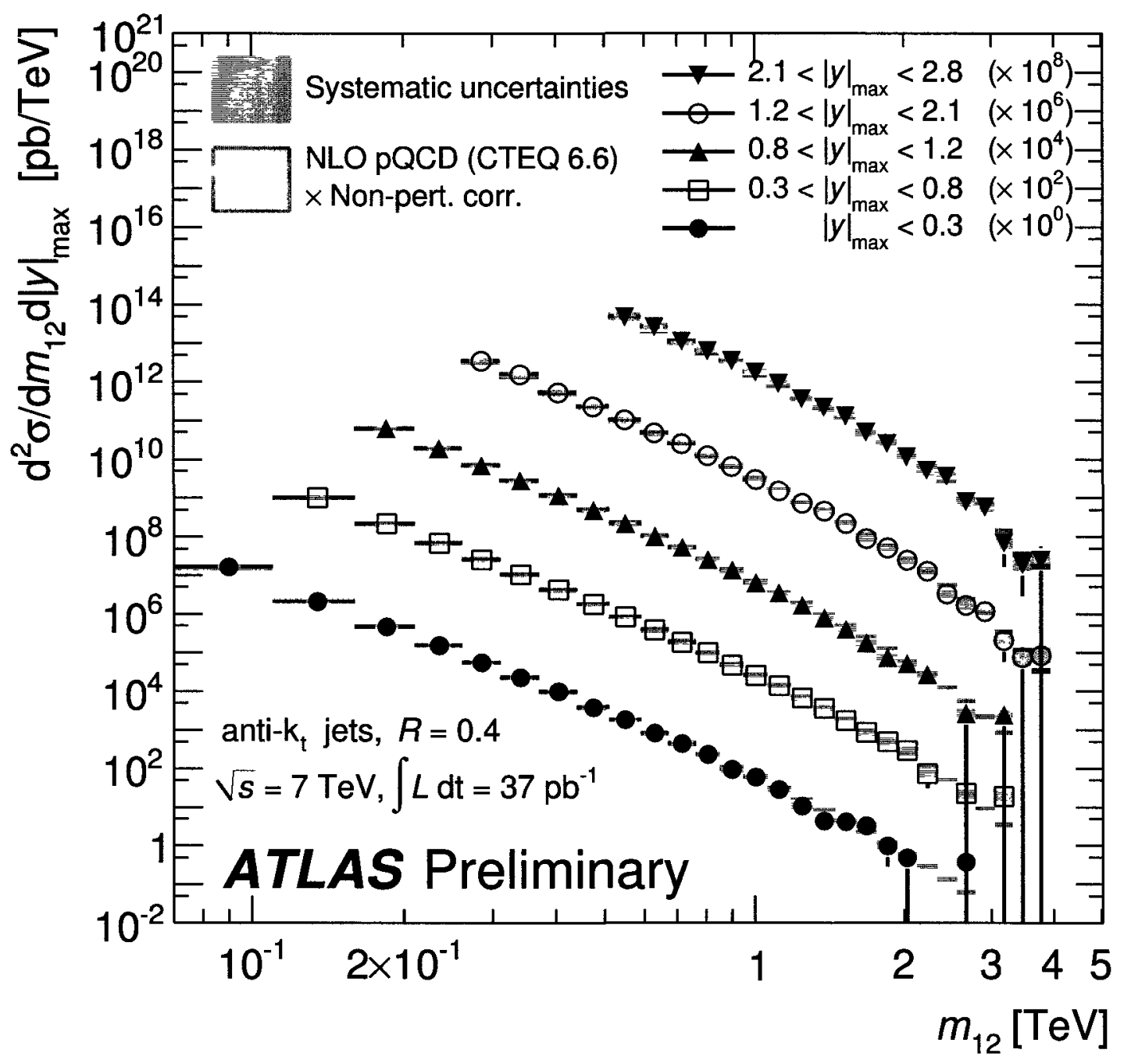

Figure 8.25: Dijet double-differential cross section as a function of dijet mass, binned in the maximum rapidity of the two leading jets $|y|_{\max }$. The results are shown for jets identified using the anti- $k_{T}$ algorithm with $R=0.4$. The data are compared to NLO pQCD calculations to which non-perturbative corrections have been applied. The systematic and theoretical uncertainties are calculated as described in Figure 8.11. 


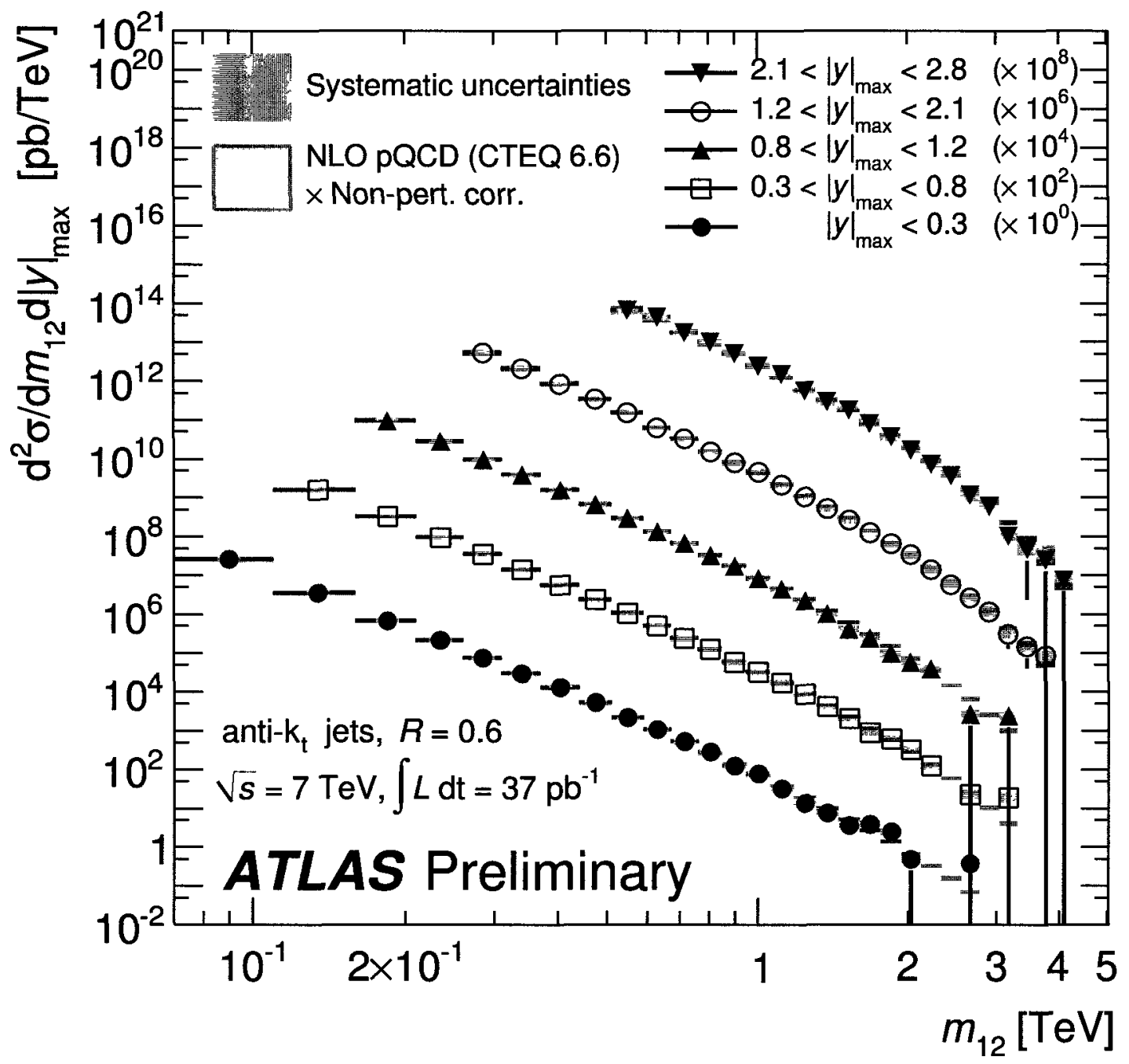

Figure 8.26: Dijet double-differential cross section as a function of dijet mass, binned in the maximum rapidity of the two leading jets $|y|_{\max }$. The results are shown for jets identified using the anti- $k_{T}$ algorithm with $R=0.6$. The data are compared to NLO pQCD calculations to which non-perturbative corrections have been applied. The systematic and theoretical uncertainties are calculated as described in Figure 8.12. 

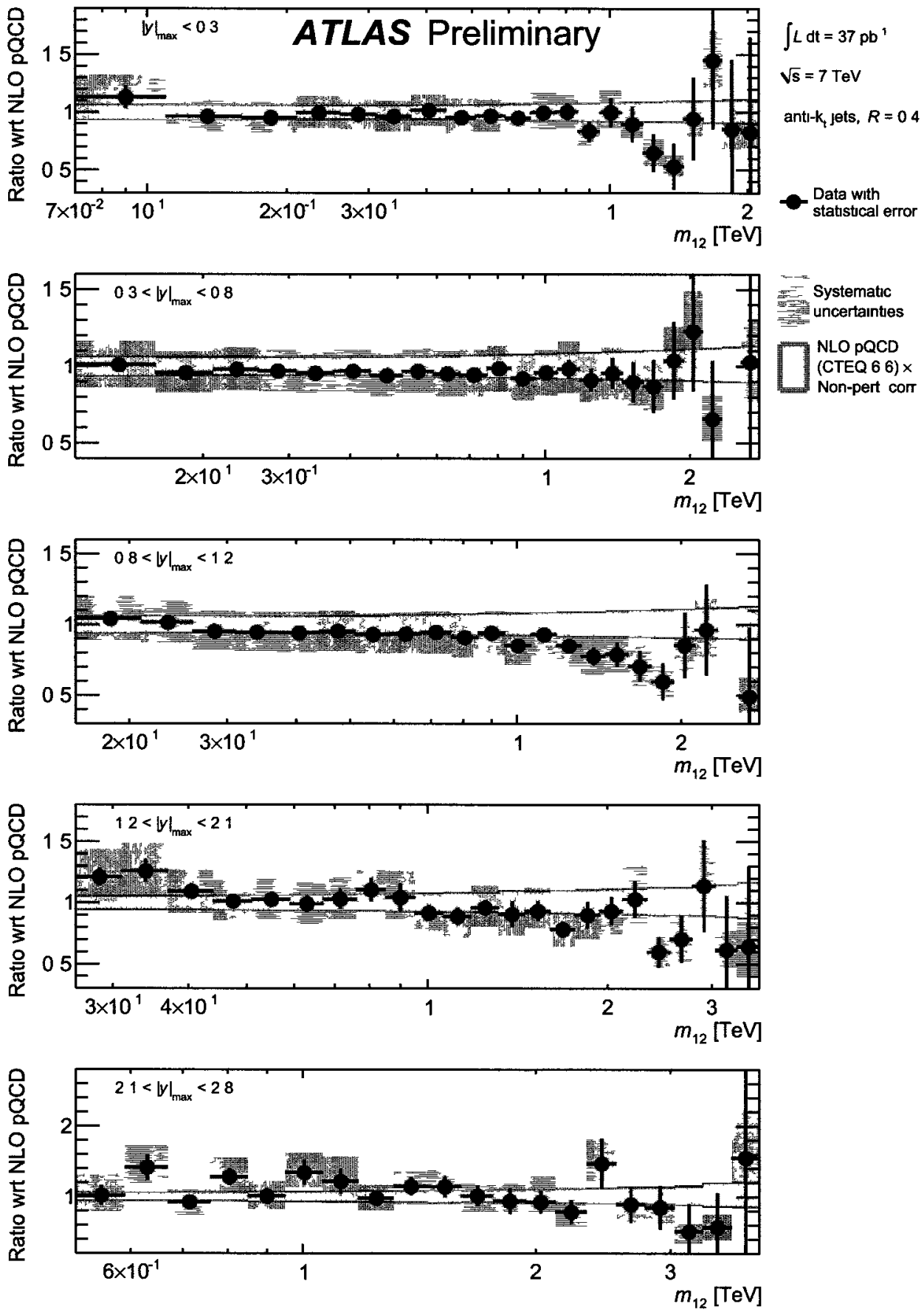

Figure 8.27. Dijet double-differential cross section as a function of dijet mass, binned in the maximum rapidity of the two leading jets $|y|_{\max }$. The results are shown for jets identified using the anti- $k_{T}$ algorithm with $R=0.4$. The data are compared to NLO pQCD calculations to which non-perturbative corrections have been applied. The systematic and theoretical uncertainties are calculated as described in Figure 8.11. 

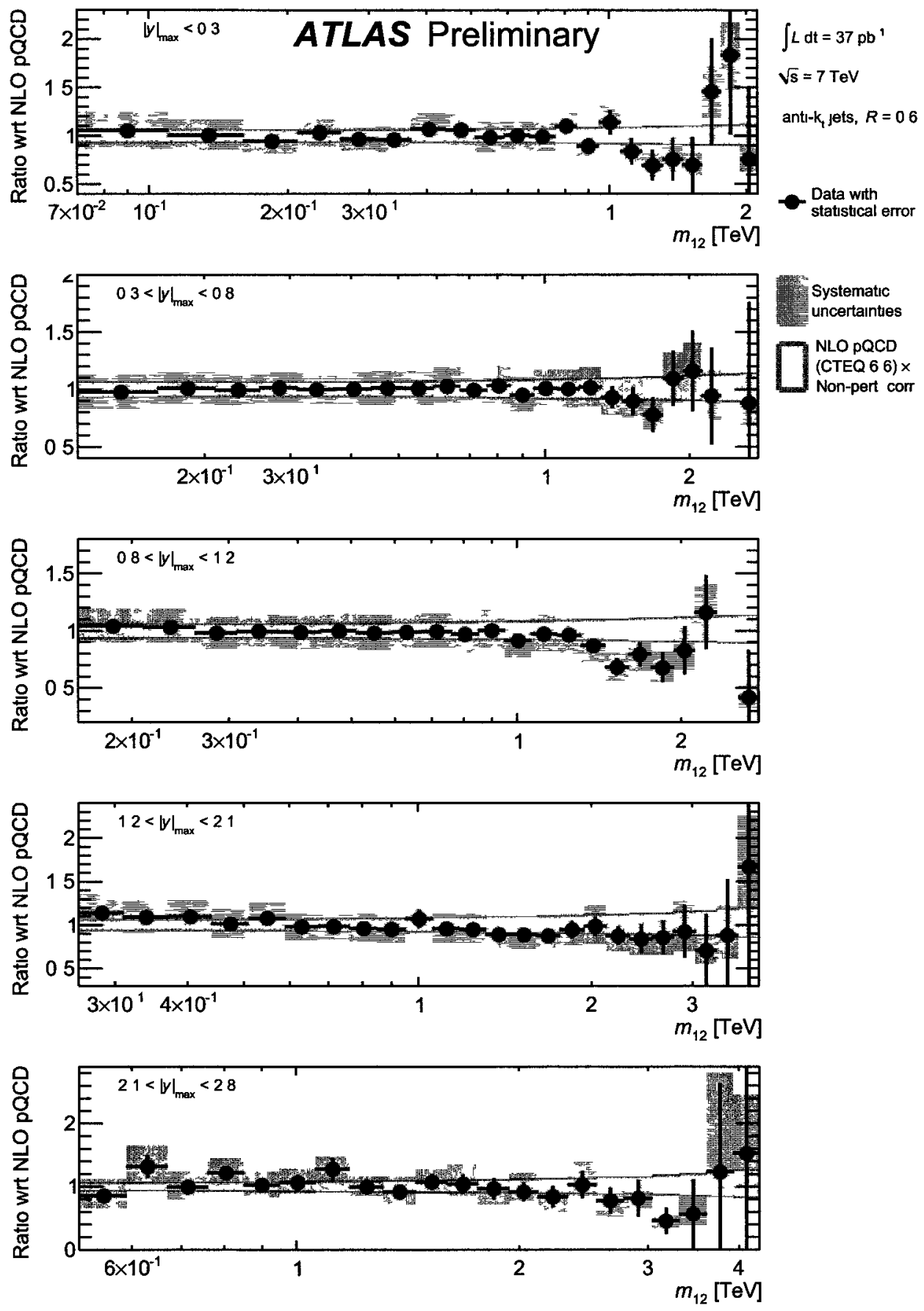

Figure 8.28: Dijet double-differential cross section as a function of dijet mass, binned in the maximum rapidity of the two leading jets $|y|_{\max }$. The results are shown for jets identified using the anti- $k_{T}$ algorithm with $R=0.6$. The data are compared to NLO pQCD calculations to which non-perturbative corrections have been applied. The systematic and theoretical uncertainties are calculated as described in Figure 8.12. 

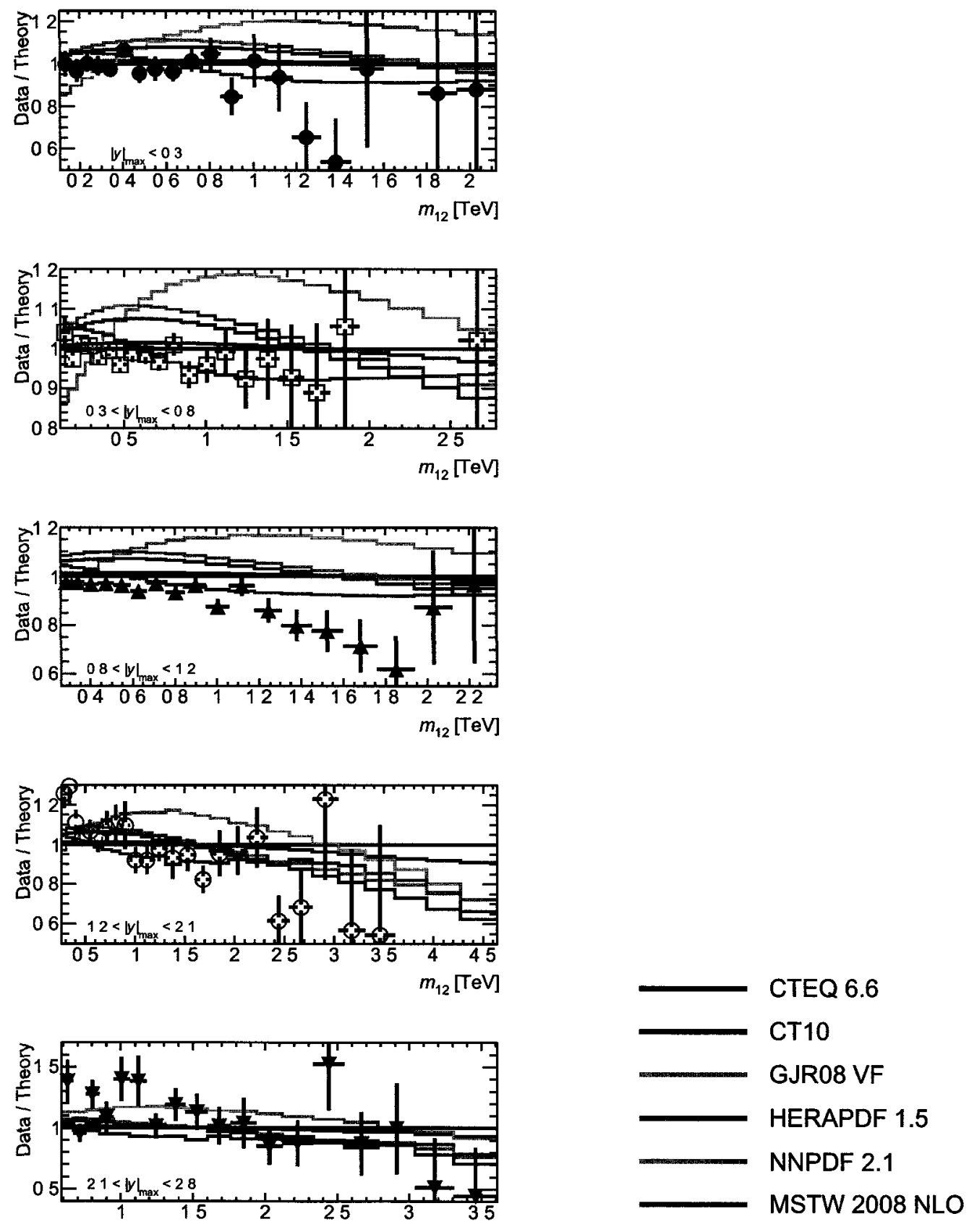

Figure 8.29: Dijet double-differential cross section as a function of dijet mass, binned in the maximum rapidity of the two leading jets $|y|_{\max }$. The results are shown for jets identified using the anti- $k_{T}$ algorithm with $R=0.4$. The data, as well as NLO pQCD predictions corrected for non-perturbative effects and using different PDF sets, are shown normalized to the theoretical prediction using the CTEQ 6.6 central value. No uncertainties are shown. For clarity, this diagram should be viewed in colour. 

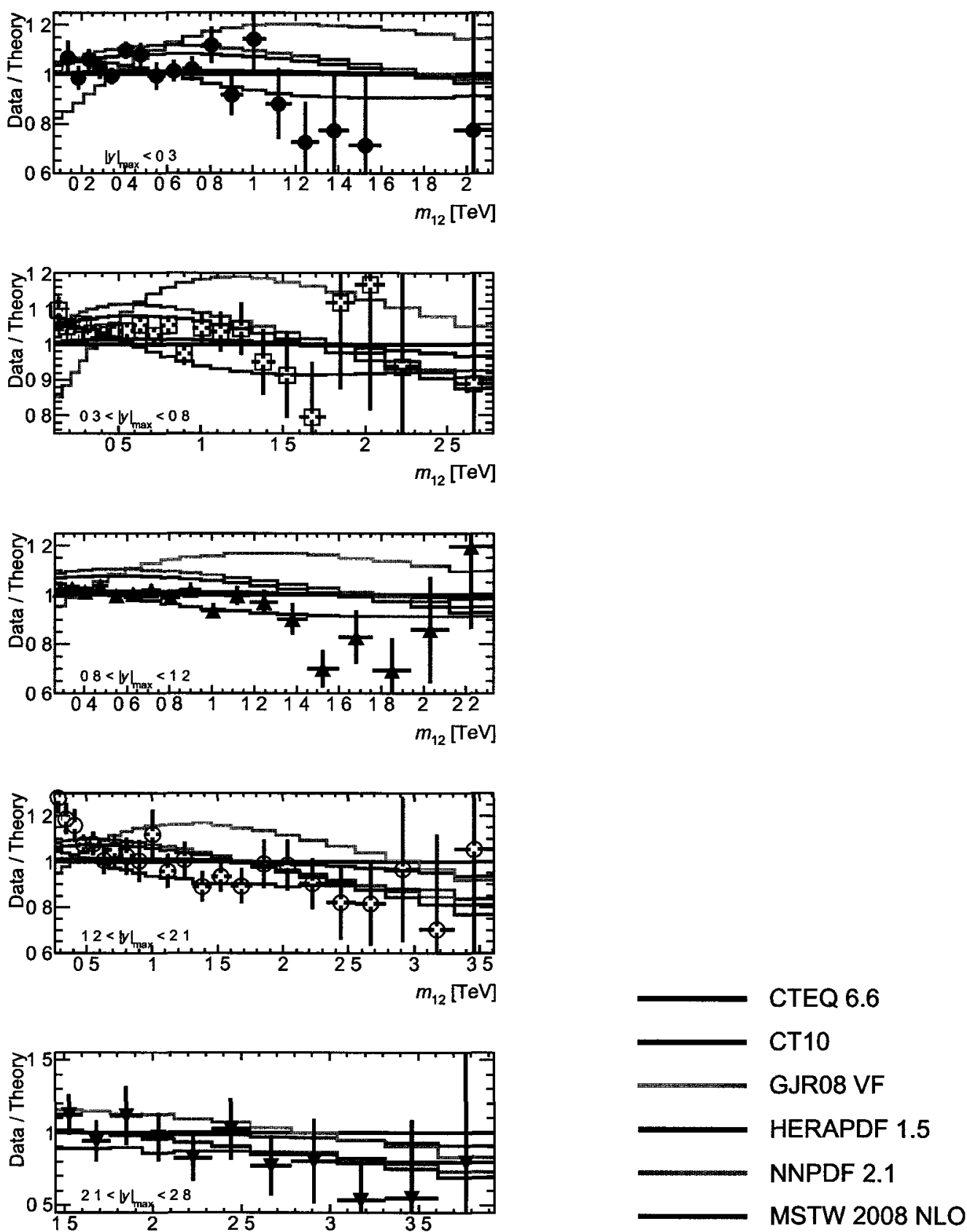

Figure 8.30: Dijet double-differential cross section as a function of dijet mass, binned in the maximum rapidity of the two leading jets $|y|_{\max }$. The results are shown for jets identified using the anti- $k_{T}$ algorithm with $R=0.6$. The data, as well as NLO pQCD predictions corrected for non-perturbative effects and using different PDF sets, are shown normalized to the theoretical prediction using the CTEQ 6.6 central value. No uncertainties are shown. For clarity, this diagram should be viewed in colour. 

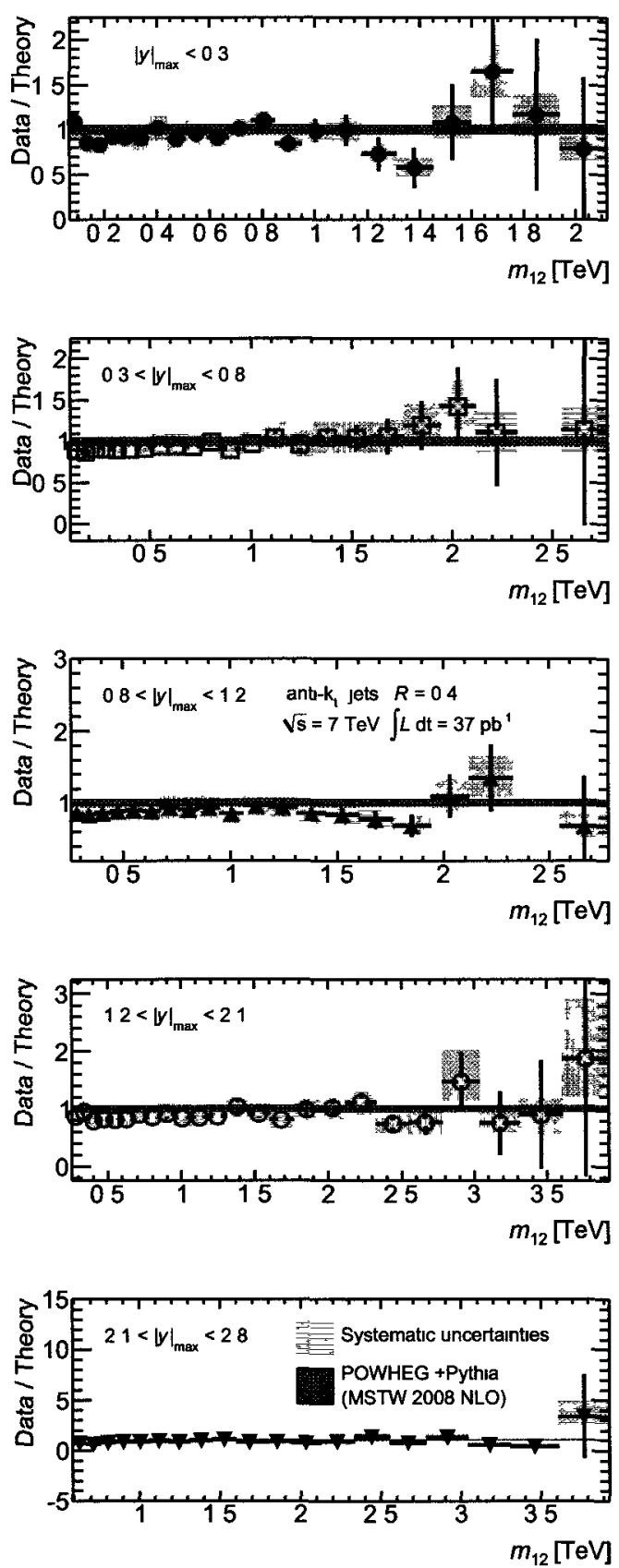

Figure 8.31: Dijet double-differential cross section as a function of dijet mass, binned in the maximum rapidity of the two leading jets $|y|_{\max }$. The results are shown for jets identified using the anti- $k_{T}$ algorithm with $R=0.4$. The data are compared to Powheg, a Monte Carlo with a NLO matrix element interfaced to a matched parton shower, hadronization, and underlying event modelled by Pythia. The experimental uncertainties are calculated as described in Figure 8.11. 

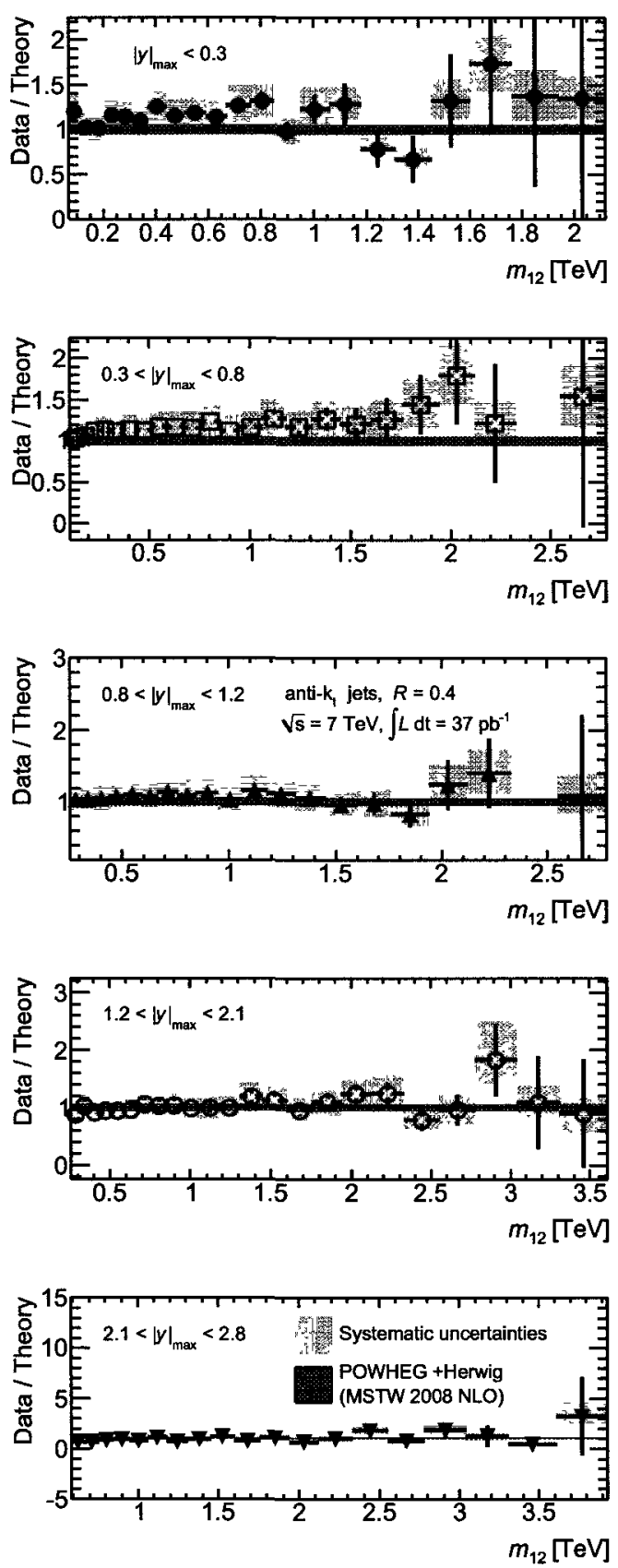

Figure 8.32: Dijet double-differential cross section as a function of dijet mass, binned in the maximum rapidity of the two leading jets $|y|_{\max }$. The results are shown for jets identified using the anti- $k_{T}$ algorithm with $R=0.4$. The data are compared to Powheg, a Monte Carlo with a NLO matrix element interfaced to a matched parton shower, hadronization, and underlying event modelled by Herwig. The experimental uncertainties are calculated as described in Figure 8.11. 

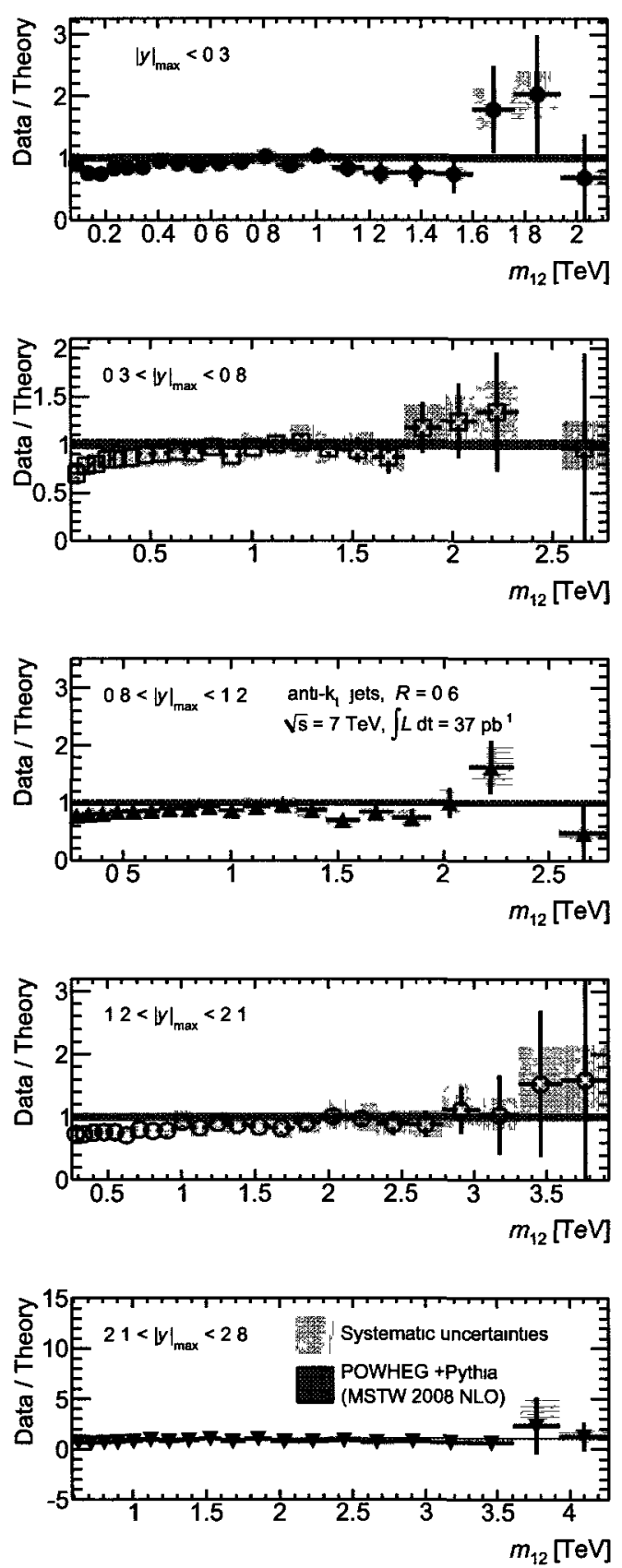

Figure 8.33: Dijet double-differential cross section as a function of dijet mass, binned in the maximum rapidity of the two leading jets $|y|_{\max }$. The results are shown for jets identified using the anti- $k_{T}$ algorithm with $R=0.6$. The data are compared to Powheg, a Monte Carlo with a NLO matrix element interfaced to a matched parton shower, hadronization, and underlying event modelled by Pythia. The experimental uncertainties are calculated as described in Figure 8.12. 

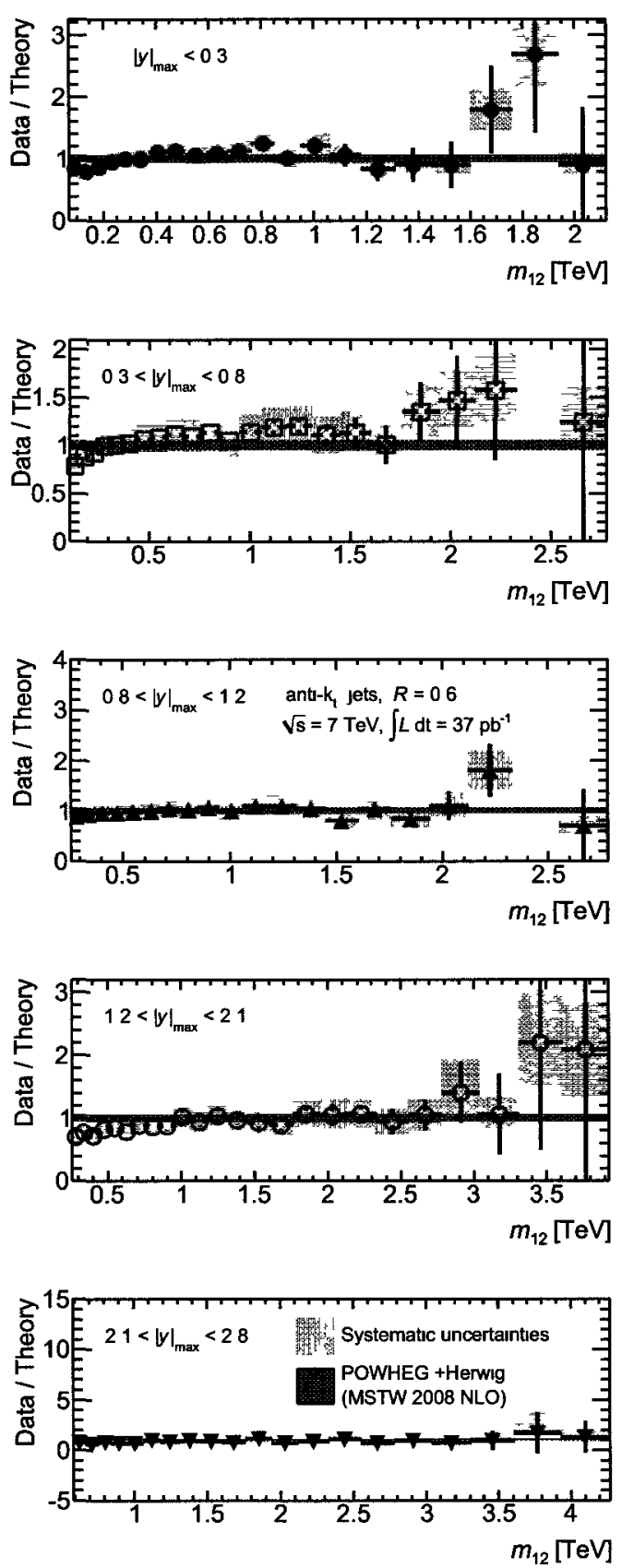

Figure 8.34: Dijet double-differential cross section as a function of dijet mass, binned in the maximum rapidity of the two leading jets $|y|_{\max }$. The results are shown for jets identified using the anti- $k_{T}$ algorithm with $R=0.6$. The data are compared to Powheg, a Monte Carlo with a NLO matrix element interfaced to a matched parton shower, hadronization, and underlying event modelled by Herwig. The experimental uncertainties are calculated as described in Figure 8.12. 


\section{Chapter 9}

\section{Summary and Conclusion}

The LHC's first run of proton-proton collisions at a center-of-mass energy of $7 \mathrm{TeV}$ has come to a successful completion, whereby $37 \mathrm{pb}^{-1}$ of data have been collected by the ATLAS detector. This early data has served both as a commissioning tool and as a window into a new and unexplored regime of physics.

The performance of the ATLAS calorimeters was assessed in-situ using the balance of transverse momentum in dijet events, where forward regions of the detector are inter-calibrated with respect to a chosen reference region. This assessment has provided an estimate of the uncertainty on jet energy measurements in the ATLAS detector. Several sources of the jet energy scale (JES) uncertainty are existent, but the source of intercalibration is the dominant one, reaching as high as $12 \%$ in the forward calorimeter (FCal). Other sources of uncertainty are added in quadrature with the intercalibration source to obtain the total JES systematic uncertainty, the largest being for low $p_{T}$ jets traversing the FCal detector, where it is approximately $13 \%$. As properties of jets are key observables of many physics analyses with the ATLAS detector, the JES uncertainty is an essential ingredient. 
The peek through a window of new physics presented in this thesis was in the area of Quantum Chromodynamics. Measurements of the inclusive jet and dijet cross sections have been presented with comparisons made to NLO pQCD theoretical predictions. This analysis probes QCD in a largely unexplored regime: highest centreof-mass collision energy ever achieved as well as the furthest reach in forward rapidities made possible by the extended coverage of the ATLAS detector, provided by FCal. For the most part, the agreement between data and theory is impressive, spanning three orders of magnitude in jet $p_{T}$ and mass, and seven orders of magnitude in cross section. As expected, the largest discrepancy observed is in the unexplored regions of phase space, i.e. very high $p_{T}$ and high mass, and forward rapidities. For the inclusive jet cross section the disagreement between data and theory is about $70 \%$ in the forward region. For the dijet cross section the disagreement is as large as $80 \%$ in the high mass domain. However, within statistical uncertainties the data agree with the theoretical predictions. As more data continue to be produced, both the statistical and systematic uncertainties will diminish and allow for more rigorous conclusions to be drawn.

The results of the physics analysis will help to constrain some of the QCD theoretical model parameters, particularly those associated with the previously unexplored regions of phase space. In addition, the measured jet rates will provide essential knowledge about the expected backgrounds for many fundamental physics analyses. The work presented here was an important first step of the LHC program and the results quoted in this thesis will be referred to extensively as the quest for fundamental physics discoveries continues at the LHC. 


\section{References}

[1] ATLAS Collaboration. Jet energy scale and its systematic uncertainty in protonproton collisions at $\sqrt{s}=7$ TeV in ATLAS 2010 data. Technical Report ATLASCONF-2011-032, CERN, Geneva, March 2011.

[2] ATLAS Collaboration. Measurement of inclusive jet and dijet cross sections in proton-proton collision data at $7 \mathrm{TeV}$ centre-of-mass energy using the ATLAS detector (Supporting Documentation). ATL-COM-PHYS-2011-140, June 2011.

[3] A. Artamonov et al. The ATLAS Forward Calorimeters. JINST, 3(P02010), Feb 2008.

[4] ATLAS Collaboration. ATLAS Detector and Physics Performance Technical Design Report. ATLAS TDR 14, CERN/LHCC 99-14, 1, May 2009.

[5] F. Halzen and A.D. Martin. Quarks and Leptons: An introductory Course in Modern Particle Physics. Wiley, New Jersey, 1987.

[6] R. Martin and G. Shaw. Introduction to Particle Physics. Cambridge University Press, Chichester, 2008.

[7] D. Griffiths. Introduction to Elementary Particles. Wiley, Weinheim, 1987.

[8] A. Breskin and R. Voss. The CERN Large Hadron Collider: Accelerator and Experiments Volumes 1 and 2. JINST 3 S08001-S08007, 2008.

[9] Particle Data Group. Review of Particle Physics. Phys. Let. B, 667, July 2008.

[10] R. Wigmans. Calorimetry: Energy Measurement in Particle Physics. Oxford Science Publications, New York, 2000. 
[11] R. Fernow. Introduction to experimental particle physics. Cambridge University Press, Cambridge, 1986.

[12] Matteo Cacciari, Gavin P. Salam, and Gregory Soyez. The anti- $k_{t}$ jet clustering algorithm. JHEP, 04:063, 2008, 0802.1189.

[13] ATLAS Collaboration. In-situ jet energy scale and jet shape corrections for multiple interactions in the first ATLAS data at the LHC. ATLAS Note, March 2011.

[14] ATLAS Collaboration. In-situ pseudorapidity intercalibration for evaluation of jet energy scale uncertainty using dijet events in proton-proton collisions at $\sqrt{s}$ $=7$ TeV. Technical Report ATLAS-CONF-2011-014, CERN, Geneva, March 2011.

[15] ATLAS Collaboration. Jet energy measurement with the ATLAS Detector in proton-proton collisions at $\sqrt{s}=7 \mathrm{TeV}$ taken in 2010. To be submitted to the European Physical Journal (EPJ), June 2011.

[16] T. Sjostrand, S. Mrenna, and P.Z. Skands. PYTHIA 6.4 Physics and Manual. $J H E P, 05: 026,2006$, hep-ph/0603175.

[17] ATLAS Collaboration. Charged particle multiplicities in pp interactions for track $p_{T}>100 \mathrm{MeV}$ at $\sqrt{s}=0.9$ and $7 \mathrm{TeV}$ measured with the ATLAS detector at the LHC. Technical Report ATLAS-CONF-2010-046, CERN, Geneva, July 2010.

[18] A. Sherstnev and R.S. Thorne. Parton distributions for LO generators. Eur. Phys. J. C, 55:553-575, 2008, 0711.2473.

[19] S. Agostinelli et al. GEANT4: A simulation toolkit. Nucl. Instrum. Meth. A, 506:250-303, 2003.

[20] M. Bahr et al. Herwig++ Physics and Manual. Eur. Phys. J., C58:639-707, 2008, 0803.0883.

[21] M.L Mangano, M. Moretti, F. Piccinini, R. Pittau, and A.D. Polosa. ALPGEN, a generator for hard multiparton processes in hadronic collisions. JHEP, 07:001, 2003, hep-ph/0206293. 
[22] P. Z. Skands. Tuning Monte Carlo Generators: The Perugia Tunes. Phys. Rev. $D, 82: 074018,2010,1005.3457$.

[23] J. Pumplin et al. New generation of parton distributions with uncertainties from global QCD analysis. JHEP, 07:012, 2002, hep-ph/0201195.

[24] G. Corcella et al. HERWIG 6.5 release note. 2002, hep-ph/0210213.

[25] J.M. Butterworth, J.R. Forshaw, and M.H. Seymour. Multiparton Interactions in Photoproduction at HERA. Z.Phys.C72:637-646, 1996, hep-ph/9601371.

[26] ATLAS Collaboration. Jet energy scale and its systematic uncertainty for jets produced in proton-proton collisions at $\sqrt{s}=7 \mathrm{TeV}$ and measured with the ATLAS detector. Technical Report ATLAS-CONF-2010-056, CERN, Geneva, July 2010.

[27] ATLAS Collaboration. ATLAS Calorimeter Response to Single Isolated Hadrons and Estimation of the Calorimeter Jet Scale Uncertainty. Technical Report ATLAS-CONF-2011-028, CERN, Geneva, March 2011.

[28] M. Aharrouche et al. Measurement of the response of the ATLAS liquid argon barrel calorimeter to electrons at the 2004 combined test- beam. Nucl. Instrum. Meth. A, 614:400-432, 2010.

[29] ATLAS Collaboration. Photon Conversions at $\sqrt{s}=900 \mathrm{GeV}$ measured with the ATLAS Detector. Technical Report ATLAS-CONF-2010-007, CERN, Geneva, Jun 2010.

[30] ATLAS Collaboration. Probing the material in front of the ATLAS electromagnetic calorimeter with energy flow from $\sqrt{s}=7 \mathrm{TeV}$ minimum bias events. Technical Report ATLAS-CONF-2010-037, CERN, Geneva, Jul 2010.

[31] ATLAS Collaboration. Study of the Material Budget in the ATLAS Inner Detector with $K_{S}^{0}$ decays in collision data at $\sqrt{s}=900 \mathrm{GeV}$. Technical Report ATLASCONF-2010-019, CERN, Geneva, Jul 2010.

[32] ATLAS Collaboration. Mapping the material in the ATLAS Inner Detector using secondary hadronic interactions in $7 \mathrm{TeV}$ collisions. Technical Report ATLASCONF-2010-058, CERN, Geneva, Jul 2010. 
[33] ATLAS Collaboration. In-situ jet energy scale and jet shape corrections for multiple interactions in the first ATLAS data at the LHC. Technical Report ATLAS-CONF-2011-030, CERN, Geneva, February 2010.

[34] J.M. Butterworth. Structure of the Photon. eConf, (C990809), Apr 2001.

[35] Z. Nagy. Next-to-leading order calculation of three jet observables in hadron hadron collision. Phys. Rev., D68:094002, 2003, hep-ph/0307268.

[36] H.-L. Lai et al. New parton distributions for collider physics. JHEP, 07:012, 2002, hep-ph/0201195.

[37] A.D. Martin, W.J. Stirling, R.S. Thorne, and G. Watt. Parton distributions for the LHC. Eur. Phys. J., C63:189-285, 2009, 0901.0002.

[38] R. D. Ball et al. A first unbiased global NLO determination of parton distributions and their uncertainties. Nucl. Phys., B838:136-206, 2010, 1002.4407.

[39] H1 Collaboration and ZEUS Collaboration. HERA Combined PDF. https://www.desy.de/h1zeus/combined_results/index.php?do=proton_structure.

[40] M. Gluck, P. Jiminez-Delgado, and E. Reya. Dynamical parton distributions of the nucleon and very small-x physics. Eur.Phys.J.C53:355-366, 2008, arXiv:0709.0614v2.

[41] Peter Z. Skands. The Perugia Tunes. 2009, arXiv: 0905.3418v1, hep-ph.

[42] A. Buckley et al. Systematic event generator tuning for the LHC. Eur. Phys. J. C, 65:331-357, 2010, 0907.2973.

[43] S. Frixione and B. R. Webber. Matching NLO QCD computations and parton shower simulations. JHEP, 06:029, 2002, hep-ph/0204244.

[44] P. Nason. A new method for combining NLO QCD with shower Monte Carlo algorithms. JHEP, 11:040, 2004, hep-ph/0409146.

[45] S. Frixione, P. Nason, and C. Oleari. Matching NLO QCD computations with Parton Shower simulations: the POWHEG method. JHEP, 11:070, 2007, 0709.2092 . 
[46] S. Frixione, P. Nason, and G. Ridolfi. The POWHEG-hvq manual version 1.0. 2007, 0707.3081.

[47] S. Alioli, P. Nason, C. Oleari, and E. Re. A general framework for implementing NLO calculations in shower Monte Carlo programs: the POWHEG BOX. 2010, arXiv: 1002.2581, hep-ph.

[48] ATLAS Collaboration. First tuning of HERWIG/JIMMY to ATLAS data. Technical Report ATL-PHYS-PUB-2010-014, CERN, Geneva, October 2010.

[49] ATLAS Collaboration. Charged-particle multiplicities in pp interactions at $\sqrt{s}=$ $900 \mathrm{GeV}$ measured with the ATLAS detector at the LHC. Phys. Lett., B688:2142, 2010, 1003.3124 .

[50] ATLAS Collaboration. Measurement of inclusive jet and dijet cross sections in proton-proton collision data at $7 \mathrm{TeV}$ centre-of-mass energy using the ATLAS detector. ATLAS-CONF-2011-047, June 2011. 